\title{
Micromachined Hemispheroidal Cavity Resonators
}

\author{
Scott R. McLelland, B. Eng., M. Eng., P. Eng.
}

A thesis submitted to the Faculty of Graduate Studies and Research in partial fulfillment of the requirements for the degree of

\section{Doctor of Philosophy}

in the Ottawa-Carleton Institute of Electrical and Computer Engineering and in the Department of Electronics, Faculty of Engineering, Carleton University

(C) S. R. McLelland 2007 


$\begin{array}{ll}\begin{array}{l}\text { Library and } \\ \text { Archives Canada }\end{array} & \begin{array}{l}\text { Bibliothèque et } \\ \text { Archives Canada }\end{array} \\ \begin{array}{l}\text { Published Heritage } \\ \text { Branch }\end{array} & \begin{array}{l}\text { Direction du } \\ \text { Patrimoine de l'édition }\end{array} \\ \begin{array}{l}\text { 395 Wellington Street } \\ \text { Ottawa ON K1A ON4 }\end{array} & \begin{array}{l}\text { 395, rue Wellington } \\ \text { Ottawa ON K1A ON4 } \\ \text { Canada }\end{array}\end{array}$

Your file Votre référence ISBN: 978-0-494-33501-7 Our file Notre référence ISBN: 978-0-494-33501-7

NOTICE:

The author has granted a nonexclusive license allowing Library and Archives Canada to reproduce, publish, archive, preserve, conserve, communicate to the public by telecommunication or on the Internet, loan, distribute and sell theses worldwide, for commercial or noncommercial purposes, in microform, paper, electronic and/or any other formats.

The author retains copyright ownership and moral rights in this thesis. Neither the thesis nor substantial extracts from it may be printed or otherwise reproduced without the author's permission.
AVIS:

L'auteur a accordé une licence non exclusive permettant à la Bibliothèque et Archives Canada de reproduire, publier, archiver, sauvegarder, conserver, transmettre au public par télécommunication ou par l'Internet, prêter, distribuer et vendre des thèses partout dans le monde, à des fins commerciales ou autres, sur support microforme, papier, électronique et/ou autres formats.

L'auteur conserve la propriété du droit d'auteur et des droits moraux qui protège cette thèse. $\mathrm{Ni}$ la thèse ni des extraits substantiels de celle-ci ne doivent être imprimés ou autrement reproduits sans son autorisation.
In compliance with the Canadian

Privacy Act some supporting forms may have been removed from this thesis.

While these forms may be included in the document page count, their removal does not represent any loss of content from the thesis.
Conformément à la loi canadienne sur la protection de la vie privée, quelques formulaires secondaires ont été enlevés de cette thèse.

Bien que ces formulaires aient inclus dans la pagination, il n'y aura aucun contenu manquant.

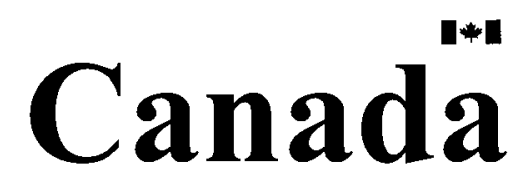




\section{Abstract}

Novel resonators with high loaded quality factors can be used to reduce oscillator phase noise. Accordingly, a micromachined oblate hemispheroidal cavity was designed, fabricated and measured to achieve the best possible loaded quality factor at W-band.

These cavities were micromachined in a silicon wafer using self-limited isotropic etching. Next they were metalized and aligned to an alumina wafer that was previously patterned with microstrip feed lines each having an aperture in the ground plane for coupling to each cavity (the ground plane forms the top of the cavities). After alignment, the silicon and the alumina were soldered together using a thin indium layer that was previously patterned on the back of the alumina. Vialess probe pads on the alumina allow wafer probing without drilled substrate vias.

A newly developed analysis based on equivalent energy aperture coupling theory and perturbation techniques was used to design the cavities. The resonant frequency, unloaded and loaded quality factors for the fully-assembled micromachined cavity can be calculated using simple equations. Results from these equations were compared to both 3D finite element simulations and measurements and they were all in good agreement.

A prototype micromachined cavity had a single measured resonance at $76.39 \mathrm{GHz}$ with a measured unloaded quality factor of 1426 and a measured loaded quality factor of 909 . This performance is better than any reported micromachined cavity and may reduce oscillator phase noise by as much as $30 \mathrm{dBc}$ at $\mathrm{W}$-band. 


\section{Acknowledgments}

This thesis would not have been possible without the support of many people. Many thanks to my supervisor, Dr. Jim Wight, and my co-supervisor, Dr. Malcolm Stubbs, for their years of advice. Also, thanks to Dr. Malcolm Stubbs, Dr. Valek Szwarc and Dr. Gerry Chan for allowing me to pursue this research at the Communications Research Centre (CRC), and to my colleagues in the IMMC group, both current and former, for their help and insights along the way. Special thanks are also due to all of the members of the Microfabrication Facility at CRC who fabricated the prototype circuits. And finally, thanks to Jen, Abby and Megan who endured this long process with me, always offering support and love. 


\section{Contents}

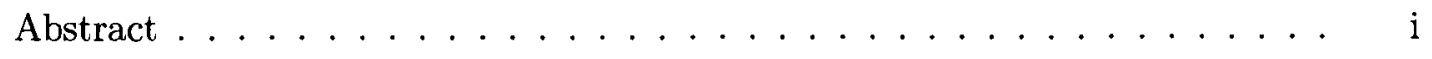

Acknowledgments ....................... ii

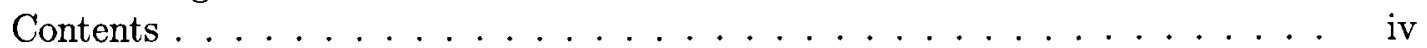

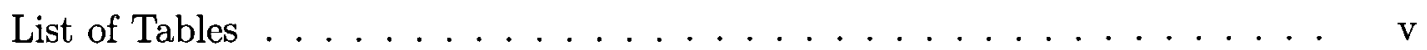

List of Figures . . . . . . . . . . . . . . . . ix

List of Symbols and Abbreviations ............... $\ldots$

1 Introduction $\quad 1$

1.1 Motivation and Overview . . . . . . . . . . . . . . . . 1

1.2 Goals and Expected Contributions . . . . . . . . . . . . 7

1.3 Thesis Organization . . . . . . . . . . . . . . . 8

2 Background $\quad \mathbf{1 0}$

2.1 Micromachined Resonators . . . . . . . . . . . . . . . . . . 10

2.2 The Perfectly Hemispherical Resonator . . . . . . . . . . . . . . 19

2.3 Isotropic Wet Etching . . . . . . . . . . . . . . 27

3 Cavity Design $\quad 30$

3.1 Resonant Frequency . . . . . . . . . . . . . . . . . . . 30

3.2 Unloaded Quality Factor . . . . . . . . . . . . . . . . 33

3.3 Unloaded Simulations . . . . . . . . . . . . . . . . . . . 37

3.4 Loaded Quality Factor . . . . . . . . . . . . . . . . . . . . . . 40

3.5 Loaded Simulations . . . . . . . . . . . . . . . . . . . . . 51

4 Cavity Fabrication $\quad \mathbf{5 6}$

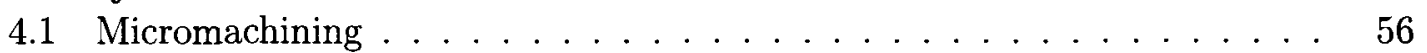

4.2 The Feed Substrate . . . . . . . . . . . . . . . . . 68

4.3 Vialess Probe Pads . . . . . . . . . . . . . . . . . . . . . . 71

4.4 Assembly . . . . . . . . . . . . . . . . . . . . 80

4.5 Final Prototype Fabrication . . . . . . . . . . . . 85

5 Cavity Performance $\quad \mathbf{8 8}$

5.1 Micromachined Cavity Measurements . . . . . . . . . . . . . . . 88

5.2 Comparison to Machined Cavities . . . . . . . . . . . 95 
5.3 Phase Noise Improvement $\ldots \ldots \ldots . \ldots . \ldots . \ldots 9$

6 Conclusion 101

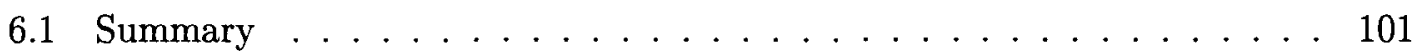

6.2 Contributions . . . . . . . . . . . . . . . . . . 104

6.3 Future Work . . . . . . . . . . . . . . . . . . . 106

6.4 Final Remarks. . . . . . . . . . . . . . . . . . 109

$\begin{array}{lr}\text { A Final Procedures and Masks } & \mathbf{1 1 0}\end{array}$

$\begin{array}{ll}\text { References } & \mathbf{1 1 5}\end{array}$ 


\section{List of Tables}

2.1 Measured performance of published micromachined cavity resonators . .

3.1 Simulated unloaded resonant frequencies and unloaded quality factors (with realistic surface resistance of 1.5 times ideal). . . . . . . . . . 38

3.2 Microstrip effective dielectric constants calculated using published methods. 47

4.1 Various masks for long duration isotopic etching of silicon using $\mathrm{HNA}(\mathrm{HF}$ : $\left.\mathrm{HNO}_{3}: \mathrm{CH}_{3} \mathrm{COOH}=2: 3: 3\right)$ at $50^{\circ} \mathrm{C} \ldots \ldots 57$

4.2 Single transition bandwidth (BW) for the vialess ground-signal-ground probe pad to microstrip transitions in Figure 4.15. All frequencies in $\mathrm{GHz}$. 76

5.1 Calculated, simulated and measured resonant frequencies versus the range of possible physical size for each of the prototype micromachined cavities. 93

5.2 Calculated, simulated and measured quality factors for each of the prototype micromachined cavities. . . . . . . . . . . . . . . 94 


\section{List of Figures}

1.1 Measured unloaded quality factors of existing micromachined cavity resonators with depths less than $1.5 \mathrm{~mm}$. . . . . . . . . . . . 4

1.2 Measured loaded quality factors of existing micromachined cavity resonators with depths less than $1.5 \mathrm{~mm} . \ldots \ldots . \ldots 4$

1.3 Proposed micromachined hemispherical cavity resonator. . . . . . . . 6

2.1 MEMS vibrating disk resonators with quality factors of 10,100 in air at $1.51 \mathrm{GHz}$ (left) and 14,600 in a vacuum at $1.21 \mathrm{GHz}$ (right). . . . . . 12

2.2 Examples of surface micromachined inductors. . . . . . . . . . . . 13

2.3 Micromachined membrane-supported microstrip oscillator . . . . . . . . . 14

2.4 Non-planar micromachined transmission line resonators. . . . . . . . . . 14

2.5 Micromachined cavity resonator . . . . . . . . . . . . . 15

2.6 Micromachined cavity oscillator schematic (left) and picture (right) . . . 16

2.7 Single-wafer micromachined cavity resonator . . . . . . . . . . . . 17

2.8 Schematic of a two-wafer micromachined cavity . . . . . . . . . . . 17

2.9 Stacked-wafer micromachined cavity resonator . . . . . . . . . . . . 18

2.10 Spherical coordinate system for a perfectly hemispherical cavity. . . . . 22

2.11 Five lowest order mode patterns for a perfectly hemispherical resonator. . 23

2.12 Theoretical $|\vec{E}|$ and $|\vec{H}|$ for a perfectly hemispherical cavity calculated using equations 2.3 to 2.8 for the $T M_{011}$ mode. . . . . . . . . 25

2.13 Fundamental mode pattern for a perfectly hemispherical resonator. . . . 26

2.14 Cavity geometries micromachined in a substrate: isotropically etched (left) and anisotropically etched (right). Substrates shown transparent in foreground to highlight the etched geometry. . . . . . . . . . . .

2.15 Cross-sectional views of existing micromachined hemispherical structures for acoustic microphones (left) and thermofusion nuclear pellets (right). .

3.1 Perturbations of a perfect hemisphere resulting in an oblate hemisphere with solder thickness. . . . . . . . . . . . . . . . 32

3.2 Theoretical surface resistance multiplier, $K$, due to roughness published by both Hoffman and Groiss/HFSS. . . . . . . . . . . . . . 36

3.3 Unloaded eigenmode simulation geometries. . . . . . . . . . . 38 
3.4 Unloaded eigenmode simulation of the $T M_{011}$ electric and magnetic fields in a perfect hemispherical cavity. . . . . . . . . . . . . . .

3.5 Unloaded eigenmode simulation of the $T M_{011}$ electric and magnetic fields in an oblate hemispheroid micromachined cavity with a solder layer. . . . 39

3.6 Equivalent circuit model for a cavity aperture coupled to microstrip. . . . 41

3.7 Aperture coupling between two microstrips and the equivalent parallel plate waveguide model. . . . . . . . . . . . . . . 47

3.8 Calculated loaded quality factor as a function of circular aperture radius. $\quad 50$

3.9 Micromachined cavity geometry for driven simulations. . . . . . . . . . 51

3.10 Simulated $S_{11}$ for micromachined cavities with aperture radii from $0.12 \mathrm{~mm}$

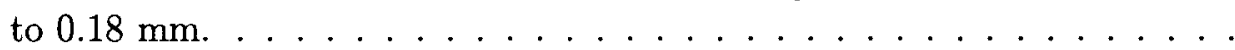

3.11 Simulated $\mathrm{S}_{11}$ showing undercoupling for micromachined cavities with aperture radii from $0.12 \mathrm{~mm}$ (smallest circle) to $0.18 \mathrm{~mm}$ (largest circle) in steps

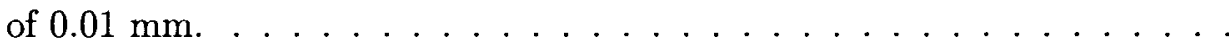

3.12 Broadband simulation of $\mathrm{S}_{11}$ for a micromachined cavity with an aperture radius of $0.18 \mathrm{~mm} . \ldots \ldots \ldots \ldots$

3.13 Simulated electromagnetic fields for a micromachined cavity with an aperture radius of $0.18 \mathrm{~mm} \ldots \ldots \ldots \ldots \ldots$

3.14 Simulated and calculated loaded quality v. aperture radius for a micromachined cavity. . . . . . . . . . . . . 55

4.1 Self-limiting of the etch rate due to gas by-products . . . . . . . . . . 59

4.2 Initial results of isotropic etching experiments. . . . . . . . . . . . 60

4.3 Patterned tape, aluminum mask and improved tape mask (left) resulting in the first repeatable cavities from isotropic etching experiments (right).

4.4 Etching experiments with (left) and without (right) agitation at $50^{\circ} \mathrm{C}$. . .

4.5 Etching experiments at $50^{\circ} \mathrm{C}$ (left) and $70^{\circ} \mathrm{C}$ (right). . . . . . . 62

4.6 General size of the etched cavities. . . . . . . . . . . . . . 62

4.7 Measured sizes of 13 etched cavity samples. . . . . . . . . . . . . 64

4.8 Measured elliptical profile for cavity 1 (left) and cavity 12 (right) of Figure $4.7 \ldots \ldots \ldots \ldots \ldots \ldots \ldots$. . . . . . . . . . . . . . . . . . . .

4.9 Sawed cavities (left) for inspecting the cross-section (right). . . . . . . . 66

4.10 Scanning electron microscope pictures of sawed cavity cross-sections that were etched at $50^{\circ} \mathrm{C}$ and $70^{\circ} \mathrm{C} \ldots \ldots \ldots 66$

4.11 Final smooth and well-shaped oblate hemispherical cavities micromachined using self-limited isotropic etching. . . . . . . . . . . . . 67

4.12 Final feed substrate. . . . . . . . . . . . . . . . . . 68

4.13 Top side of an early alumina feed substrate using drilled vias for the coplanar probe pad to microstrip transition. . . . . . . . . . . . . . .

4.14 Method of moments simulation showing that the ground-signal-ground probe pad to microstrip transition, using drilled vias, only works up to about $30 \mathrm{GHz}$. 
4.15 Test substrate of a number of different vialess transitions from groundsignal-ground probe pads to microstrip. . . . . . . . . . . .

4.16 Measurements and simulations of straight stub vialess probe pads from the vialess test wafer in Figure 4.15. . . . . . . . . . . . . . . .

4.17 Measurements and simulations of radial stub vialess probe pads from the vialess test wafer in Figure 4.15. . . . . . . . . . . . .

4.18 Measurements and simulations of merged radial stub vialess probe pads from the vialess test wafer in Figure $4.15 \ldots \ldots \ldots \ldots . . . \ldots$

4.19 Simulation of a single $77 \mathrm{GHz}$ vialess probe pad designed for micromachined cavity measurements $\left(90^{\circ}\right.$ merged radial stubs, see Figure 4.12$)$. .

4.20 Measurements and simulations of the TRL verification line with OSL calibration at the probe tips to test the back-to-back vialess transition used for cavity measurements. . . . . . . . . . . . . . . .

4.21 Measurement of $50 \Omega$ verification line on final cavity feed substrate after vialess probe pads are de-embedded using TRL calibration. . . . . . . .

4.22 Gold-tin-gold soldering experiments. . . . . . . . . . . . . . . . 81

4.23 Results of initial indium plating development. . . . . . . . . . . . . 82

4.24 Results of final indium plating. . . . . . . . . . . . . . . . . . . . . 82

4.25 Results of patterned indium solder layer. . . . . . . . . . . . . 83

4.26 Exposed cavity top surface (gold microstrip ground plane), aperture and alignment marks after indium solder layer patterning. . . . . . . . . 8 83

4.27 Cross-section of a micromachined cavity soldered to an alumina feed wafer using indium. . . . . . . . . . . . . . . .

4.28 Prototype micromachined cavities for testing (after etching but before soldering). . . . . . . . . . . . . . . . 86

4.29 Fully-assembled prototype micromachined cavities ready for testing. . . . 86

5.1 Measured $S_{11}$ for micromachined prototype cavity $1 \ldots \ldots \ldots$

5.2 Measured $S_{11}$ for micromachined prototype cavity $2 \ldots \ldots \ldots$. . . 90

5.3 Measured $S_{11}$ for micromachined prototype cavity 3. . . . . . . . . 91

5.4 Measured $S_{11}$ for micromachined prototype cavity $4 \ldots \ldots \ldots$. . . . . . 91

5.5 Measured $S_{11}$ for micromachined prototype cavities 1 to 4 . . . . . . 92

5.6 Simulated $S_{11}$ using the actual size and loss for prototype micromachined cavity 1 along with its corresponding measured $S_{11} \ldots \ldots . \ldots 95$

5.7 Machined brass cavities ready for assembly. . . . . . . . . . . . 96

5.8 Assembled brass cavities. . . . . . . . . . . . . . . . . 96

5.9 Measured $\mathrm{S}_{11}$ of an oblate hemispherical cavity fabricated in brass using traditional machining. . . . . . . . . . . . . . . . .

5.10 Phase noise decrease when a conventional resonator is replaced with a resonator having $\mathrm{X}$ times higher loaded quality factor, such as a prototype

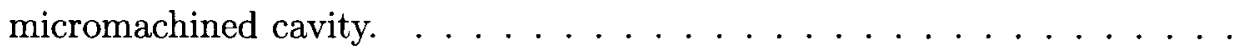


6.1 Measured unloaded quality factors of the prototype micromachined cavities compared with existing micromachined cavity resonators. . . . . . . 105

6.2 Measured loaded quality factors of the prototype micromachined cavities compared with existing micromachined cavity resonators. . . . . . . . 105

6.3 A possible micromachined spherical cavity resonator, although an actual cavity is likely to be more ellipsoidal. . . . . . . . . . . . . 108 


\section{List of Symbols and Abbreviations}

\begin{tabular}{|c|c|}
\hline$\S$ & Section \\
\hline$\alpha$ & Attenuation \\
\hline$\beta$ & Propagation constant \\
\hline$\Delta \omega$ & Offset from the carrier \\
\hline$\Delta \omega_{1 / f^{3}}$ & Flicker corner frequency \\
\hline$\Delta \tau$ & Volume perturbation \\
\hline$\delta_{s}$ & Skin depth \\
\hline$\epsilon$ & Permittivity \\
\hline$\epsilon_{e f f, d c}$ & Quasi-static effective dielectric constant \\
\hline$\epsilon_{e f f}$ & Effective dielectric constant with dispersion \\
\hline$\epsilon_{o}$ & Permittivity of free space \\
\hline$\epsilon_{r}$ & Relative permittivity \\
\hline$\eta$ & Intrinsic impedance of free space \\
\hline$\lambda$ & Wavelength \\
\hline$\lambda_{g}$ & Guided wavelength \\
\hline$\lambda_{o}$ & Free space wavelength \\
\hline $\mathcal{L}$ & Phase noise \\
\hline $\mathcal{R}$ & Surface resistance \\
\hline $\mathrm{Al}$ & Aluminum \\
\hline $\mathrm{Au}$ & Gold \\
\hline $\mathrm{CF}_{4}$ & Carbon tetrafluoride \\
\hline $\mathrm{CH}_{3} \mathrm{COOH}$ & Acetic acid \\
\hline $\mathrm{Cr}$ & Chromium \\
\hline $\mathrm{H}_{2} \mathrm{O}$ & Water \\
\hline $\mathrm{HF}$ & Hydrofluoric acid \\
\hline $\mathrm{HNO}_{3}$ & Nitric acid \\
\hline In & Indium \\
\hline $\mathrm{Si}_{3} \mathrm{~N}_{4}, \mathrm{SiN}$ & Silicon nitride \\
\hline $\mathrm{SiO}_{2}$ & Silicon dioxide \\
\hline $\mathrm{Ti}$ & Titanium \\
\hline$\mu$ & Permeability \\
\hline$\mu_{o}$ & Permeability of free space \\
\hline$\mu_{r}$ & Relative permeability \\
\hline
\end{tabular}




\begin{tabular}{|c|c|}
\hline$\omega$ & Angular frequency \\
\hline$\omega_{o}$ & Carrier angular frequency \\
\hline$\psi$ & Surface roughness \\
\hline$\sigma$ & Conductivity \\
\hline $\tan \delta$ & Loss tangent \\
\hline$\tau_{o}$ & Volume of an unperturbed perfect hemisphere \\
\hline$\vec{E}$ & Electric field \\
\hline$\vec{H}$ & Magnetic field \\
\hline$\vec{H}_{a p}$ & Tangential magnetic field at the aperture location \\
\hline$a$ & Cavity lateral radius \\
\hline$c$ & Cavity depth \\
\hline$e$ & Eccentricity \\
\hline$F$ & Oscillator noise factor \\
\hline$f_{o}$ & Frequency \\
\hline$h$ & Solder thickness \\
\hline$h_{\mu s t r i p}$ & Microstrip substrate thickness \\
\hline$k$ & Cavity to cavity coupling coefficient \\
\hline$k_{o}$ & Wavenumber \\
\hline$k T$ & Boltzman constant multiplied by temperature \\
\hline$K$ & Surface resistance multiplication factor \\
\hline $\mathcal{K}$ & Constant \\
\hline$l$ & Length \\
\hline$M$ & Magnetic polarizability \\
\hline$P$ & Electric polarizability \\
\hline$P_{s i g}$ & Oscillator power \\
\hline$Q$ & Quality factor \\
\hline$Q_{l}$ & Loaded quality factor \\
\hline$Q_{o}$ & Unloaded quality factor \\
\hline$r_{\max }$ & Aperture location \\
\hline$r_{a}$ & Aperture radius \\
\hline$S_{\text {oh }}$ & Surface area of an oblate hemisphere \\
\hline$S_{o}$ & Surface area of a perfect hemisphere \\
\hline$S$ & Normalizing factor \\
\hline$T$ & Transmission coefficient \\
\hline$T E_{m n p}$ & Transverse electric mode \\
\hline$T M_{m n p}$ & Transverse magnetic mode \\
\hline$T E M$ & Transverse electromagnetic mode \\
\hline$u_{n p}$ & Ordered zero of the spherical Bessel function \\
\hline$u_{n p}^{\prime}$ & Ordered zero of the derivative of the spherical Bessel function \\
\hline$V_{a p}$ & Aperture effective volume \\
\hline$V_{c a v}$ & Cavity effective volume \\
\hline$V_{o h}$ & Volume of an oblate hemisphere \\
\hline
\end{tabular}




$\begin{aligned} V_{o} & \text { Volume of a perfect hemisphere } \\ w_{\mu s t r i p} & \text { Microstrip width } \\ W_{\text {eff }} & \text { Effective width of PPWG model for microstrip } \\ x & \text { Normalized aperture reactance } \\ Z_{\text {in }} & \text { Input impedance } \\ Z_{o} & \text { Characteristic impedance } \\ \text { rms } & \text { Root mean square } \\ \text { BW } & \text { Bandwidth } \\ \text { CPW } & \text { Coplanar waveguide } \\ \text { CRC } & \text { Communications Research Centre } \\ \text { CMOS } & \text { Complementary metal oxide semiconductor } \\ \text { GaAs } & \text { Gallium arsenide } \\ \text { GSG } & \text { Ground-signal-ground } \\ \text { HFSS } & \text { High frequency structure simulator } \\ \text { HNA } & \text { Etchant using hydrofluoric acid, nitric acid, and, water or acetic acid } \\ \text { IC } & \text { Integrated circuit } \\ \text { LC } & \text { Inductor-capacitor } \\ \text { LTCC } & \text { Low-temperature co-fired ceramic } \\ \text { MEMS } & \text { Microelectromechanical systems } \\ \text { MMIC } & \text { Monolithic microwave integrated circuit } \\ \text { OSL } & \text { Open-short-load } \\ \text { PPWG } & \text { Parallel plate waveguide } \\ \text { PECVD } & \text { Plasma Enhanced Chemical Vapor Deposition } \\ \text { RIE } & \text { Reactive ion etching } \\ \text { SiGe } & \text { Silicon germanium } \\ \text { SEM } & \text { Scanning electron microscope } \\ \text { TRL } & \text { Through-reflect-line } \\ & \\ & \end{aligned}$




\section{Chapter 1}

\section{Introduction}

\subsection{Motivation and Overview}

Despite recent advances in oscillator design, all oscillators will have some random fluctuation in their output frequency. This frequency fluctuation is known as oscillator phase noise, and because of it, millimeter wave oscillators are not capable of generating a single, stable output frequency. This can degrade the performance of radar and communications systems that depend on a millimeter wave oscillator to produce a stable carrier frequency. Thus, minimizing oscillator phase noise is very important for good system performance.

One of the most well-known techniques to reduce oscillator phase noise is to use a resonator with a high loaded quality factor within the oscillator [1]. The "loaded quality factor" is the quality factor of the resonator when it is connected to an external load (as opposed to the "unloaded quality factor" of the resonator by itself). The higher the loaded quality factor, $Q_{l}$, of the resonator the higher its frequency selectivity when used in the feedback path of an oscillator. This increases the oscillator stability and thus lowers the phase noise. In fact, the loaded quality factor has been called both a "silver bullet" and the "most effective design parameter for improving oscillator phase noise" [2]. 
At W-band (75-110 GHz), high resonator quality factors have yet to be achieved [3][9], especially if the oscillator is implemented in CMOS or SiGe where the loss of the lowresistivity silicon substrate can severely limit the achievable quality factor. For example, a recently reported, state-of-the-art, $75 \mathrm{GHz}$ transmission line resonator in $0.18 \mu \mathrm{m}$ CMOS has a measured loaded quality factor of only 38 [10]. Slightly higher quality factors can be achieved using transmission line resonators on lower loss substrates like alumina or GaAs, however, the quality factors are still limited to a few hundred [11, 12]. Alternatively, low frequency resonators, such as dielectric, crystal or atomic resonators, can be multiplied up to $\mathrm{W}$-band however this severely degrades their performance. Recently, low temperature co-fired ceramic (LTCC) cavity resonators have been reported with encouraging quality factors up to $30 \mathrm{GHz}[13,14]$, however, none have been reported at W-band. Clearly, for all types of existing resonators, improving the loaded quality factor remains a challenge at W-band.

Microelectromechanical systems (MEMS) is a new approach that may achieve higher loaded quality factors at W-band by overcoming the limitations of existing resonators. MEMS is a technology that uses modified integrated circuit fabrication techniques, known as micromachining, to produce mechanical or other physical structures. These structures are similar in size to, and can be integrated with, conventional electrical integrated circuits. With these new fabrication techniques, many new circuit elements are possible that may dramatically enhance the performance of millimetre wave integrated circuits. One such new circuit element may be integrated, W-band resonators with higher loaded quality factors than are currently achievable using conventional approaches.

Existing MEMS resonators, described in greater detail in Section 2.1, have not been reported at W-band, however, at lower frequencies micromachined versions of traditional resonators have been demonstrated with excellent quality factors. For example, micromachined spiral inductors and micromachined transmission line resonators have twice 
the quality factors compared to their non-micromachined counterparts. Even better, at just above $1 \mathrm{GHz}$ micromachined vibrating disk resonators are capable of quality factors in excess of 10,000 . Unfortunately all of these types of micromachined resonators are limited to frequencies much lower than W-band.

One type of MEMS resonator, the micromachined cavity resonator, is, however, quite suited to W-band although none have been reported thus far. Nevertheless, below Wband the published results for micromachined cavities are quite encouraging as shown in Figures 1.1 and 1.2. These existing micromachined cavities are described in detail in Section 2.1. They are typically etched from a semiconductor substrate, such as silicon, to form a cavity resonator that operates according to the same principles as waveguide cavities. Accordingly, micromachined cavities have higher quality factors, at higher frequencies, than micromachined inductors and transmission lines, just like traditional waveguide cavities when compared to conventional inductors and transmission lines. And, just like waveguide cavities, micromachined cavities are excellent candidates for high quality factor resonators operating at W-band and beyond.

One possible disadvantage of existing micromachined cavities is their size. They are typically larger than other types of micromachined resonators, however, since they are etched in the semiconductor substrate, the area about the cavities may be used for the rest of the oscillator circuit or for other parts of the system. Therefore, in some applications the size may not be an issue. For example, in 2007 , a $10 \mathrm{GHz}$ integrated CMOS receiver including a micromachined cavity was published that used the area above the cavity to locate the antenna [15].

Despite the success of existing micromachined cavities, two issues remain. First, none have been published operating at W-band. Second, their loaded quality factors are not optimal and can be improved upon, even at W-band. The goal of this research is to overcome these two issues. 


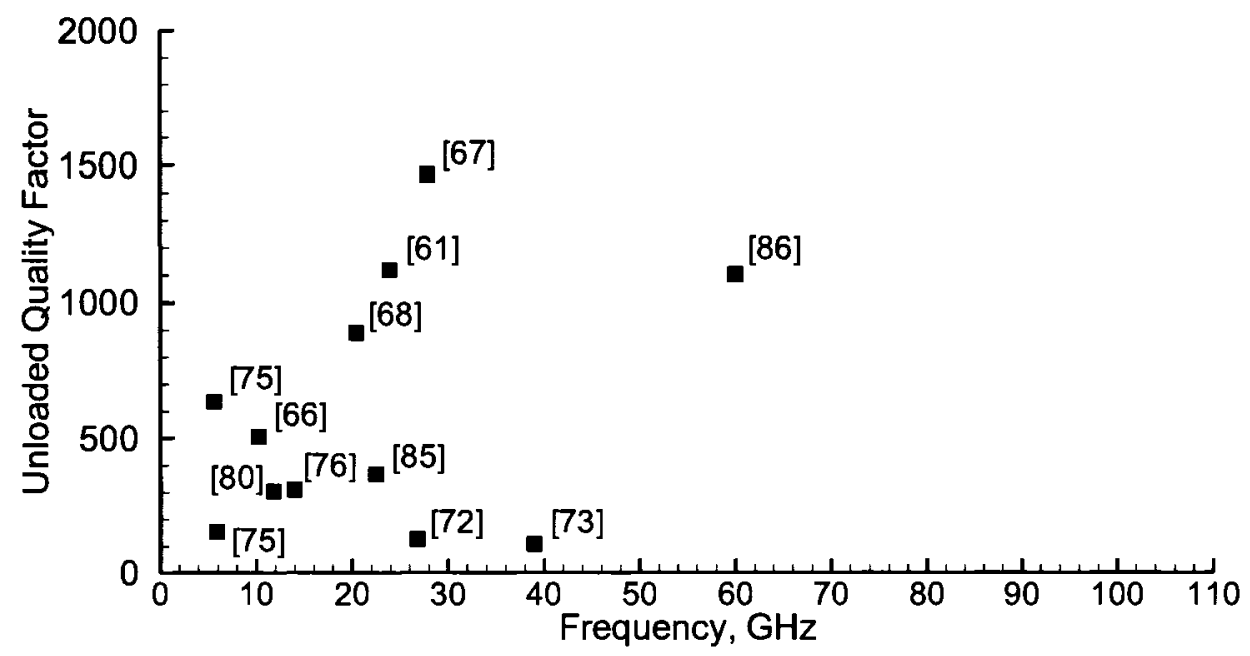

Figure 1.1: Measured unloaded quality factors of existing micromachined cavity resonators with depths less than $1.5 \mathrm{~mm}$.

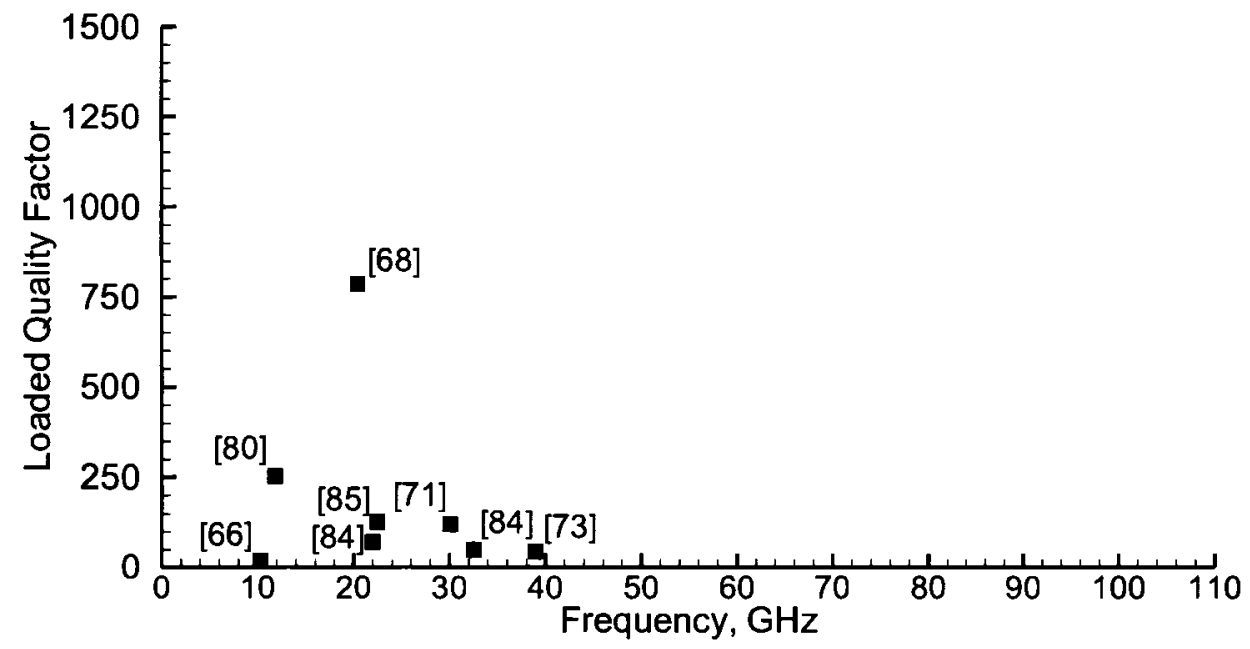

Figure 1.2: Measured loaded quality factors of existing micromachined cavity resonators with depths less than $1.5 \mathrm{~mm}$. 
First, achieving resonant frequencies at W-band is conceptually straightforward but difficult in practice. In theory, reducing the size of a micromachined cavity will increase the resonant frequency, just as in a waveguide cavity. The difficulty, however, is that as the frequency increases the wavelength decreases and imperfections in the geometry will degrade the performance much more so than at lower frequencies. At W-band, the impact of these imperfections must be investigated and care must be taken to minimize them.

Second, optimizing the loaded quality factor is important because existing micromachined cavities can be improved upon. Existing micromachined cavities, such as those described in Section 2.1, have focused solely on the unloaded quality factor. No rigorous analysis has been published to design micromachined cavities for high loaded quality factor. Furthermore, the shape of existing micromachined cavities is essentially rectangular, however, similarly sized spherical geometries are known to have higher quality factors $[16,17]$.

A spherical-type micromachined cavity, targeted towards maximizing the loaded quality factor at $\mathrm{W}$-band, was therefore chosen to overcome the limitations of existing micromachined cavities. Specifically, a hemispherical micromachined cavity was selected. It is well known that isotropically etching a silicon wafer can produce a hemispherical geometry, and this is further explained in Section 2.3. Applying isotropic etching to micromachining a cavity resonator is, however, new. In the past, isotropic etching has not been applied to micromachining cavities because controlling the size of the geometry was too difficult to accurately make a cavity. By importing a new technique, self-limited etching, from the field of micromachined acoustic microphones this problem is alleviated somewhat making isotropically etched cavities worthy of investigation. As will be seen in Section 4.1, self-limited etching can be used to define the cavity size regardless of the etching time. 


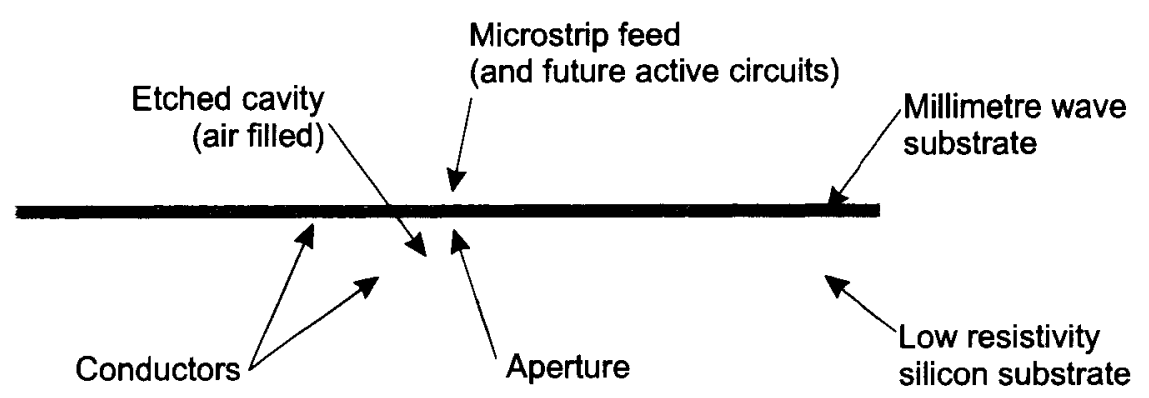

Figure 1.3: Proposed micromachined hemispherical cavity resonator.

A schematic of the novel hemispherical micromachined cavity is shown in Figure 1.3. The hemispherical cavity can be micromachined from a silicon substrate using isotropic etching and then metalized to contain the electromagnetic field. In fact, since no electromagnetic energy propagates in the silicon a low resistivity substrate can be used, thus minimizing the substrate cost. Any suitable millimetre wave substrate with a metalized bottom side can then be bonded to the etched silicon to close the hemispherical cavity. A microstrip feed transmission line on the millimetre wave substrate, with an aperture in its ground plane, will allow energy to couple into, and out of, the micromachined cavity. If the micromachined cavity resonator is used in an oscillator, then the oscillator's active and passive elements would be located on the top side of the millimetre wave substrate using the microstrip feed to connect to the cavity.

If the geometry of an isotropically etched cavity was an ideal hemisphere, then the resonant frequency, unloaded and loaded quality factors can be easily calculated using known hemispherical waveguide cavity theory presented in Section 2.2. In reality, however, the geometry is not perfectly hemispherical and thus the known theory must be modified to predict the performance of the actual cavities. The resulting new analysis, described in Chapter 3, can not only predict the performance of the actual micromachined cavities but can also be used to optimize the loaded quality factor directly.

The result of this research effort is, therefore, isotropically etched hemispherical cav- 
ities with optimized loaded quality factors at W-band. This novel type of resonator can then be applied to lowering the phase noise of $\mathrm{W}$-band oscillators as stated at the beginning of this Introduction.

\subsection{Goals and Expected Contributions}

The primary goal of this research is to produce a micromachined hemispherical cavity resonator, at W-band, with a higher loaded quality factor than existing micromachined cavities (even though these operate at lower frequencies). Also, it is desirable to develop a simple design approach that produces a specific loaded quality factor since some oscillators may be optimized if a specific loaded quality factor is used [18].

These goals will require the following contributions to be made:

- Design a novel resonator structure

- First hemispherical micromachined cavity resonator

- Custom micromachining and assembly procedure

- New vialess probe pads for testing

- Achieve the highest performance

- Highest frequency of any micromachined cavity resonator

- Highest quality factors of any micromachined cavity resonator

- Higher quality factor than conventional integrated W-band resonators

- Develop a novel design method

- New equations for the resonant frequency, the unloaded and the loaded quality factors for a micromachined hemispherical cavity 
- Capability to optimize the loaded quality factor using a simple design curve that is solely a function of aperture radius

- Aperture coupling theory for microstrip to hemispherical cavity

- Finite-elements simulations of the micromachined hemispherical cavity

These contributions are described in detail in the sections and chapters that follow.

\subsection{Thesis Organization}

The background information alluded to in Section 1.1 is described in more detail in Chapter 2. This includes reported micromachined resonators $(\S 2.1)$, existing hemispherical cavity theory $(\S 2.2)$ and known isotropic etching theory $(\S 2.3)$. These three sections serve as a basis for the actual design and fabrication of the new micromachined cavity resonators.

The design of a micromachined oblate hemispherical cavity is described in Chapter 3. First, a cavity is considered alone and equations are derived for the resonant frequency using perturbation techniques ( $(3.1)$ and also for the unloaded quality factor using a new approach that has been developed with volume and surface area ratios (§3.2). These calculated values are then verified using unloaded finite element simulations ( $\S 3.3)$. Then a cavity that is aperture coupled to microstrip is considered and equations for the loaded quality factor are derived using equivalent energy aperture coupling (§ 3.4). Finally, the calculated loaded quality factor is verified using loaded finite element simulations ( $§ 3.5$ ).

The fabrication of prototype micromachined cavities is described in Chapter 4. First, an isotropic etching procedure is developed to micromachine a cavity as close to a perfect hemispherical design as possible ( $(4.1)$. A millimetre wave substrate is also designed with a microstrip transmission line feed and a ground-plane aperture to couple energy 
into, and out of, a micromachined cavity $(\S 4.2)$. This millimeter wave substrate also includes novel vialess probe pads for measuring the cavities $(\S 4.3)$. Then an alignment and soldering procedure is developed to bond the millimetre wave substrate to the top of the micromachined silicon cavity to seal each cavity $(\S 4.4)$. These steps culminate with fabricated prototypes ready for testing ( $\S 4.5)$.

The measured performance of the prototype micromachined cavities is presented in Chapter $5(\S 5.1)$. This also includes a comparison to both the calculated and simulated performance in Chapter 3. The prototypes are further compared to conventional cavities machined in brass $(\S 5.2)$ and to existing $\mathrm{W}$-band resonators $(\S 5.3)$. The latter comparison includes the possible oscillator phase noise improvement from using the prototype micromachined cavities in place of these existing W-band resonators.

Finally, Chapter 6 summarizes the research investigation including a discussion of the results ( $\S 6.1$ ), contributions $(\S 6.2)$ and suggestions for possible future development $(\S 6.3)$. 


\section{Chapter 2}

\section{Background}

This chapter provides the relevant background and literature pertaining to micromachining hemispherical cavity resonators. First, further details are provided on the existing micromachined resonators introduced in Chapter 1, thus allowing the new micromachined hemispherical cavities to be examined in context. Next, the theoretical basis for a perfectly hemispherical resonator is explained as this is the starting point from which the micromachined hemispherical cavities are designed. Finally, isotropic etching theory is explained since this is the starting point from which the micromachined hemispherical cavities are fabricated.

\subsection{Micromachined Resonators}

Most of the literature on micromachining uses silicon as the base material. In fact the mechanical properties of silicon have been well documented as early as 1982 [19]. Since then, MEMS have been successfully used to enhance the performance of many products, such as accelerometers that control airbags and ink jet nozzles in printers. The application of MEMS at RF and microwave frequencies is more recent but the promise 
of better performance is no less. Specifically, RF MEMS have provided RF switches, variable capacitors and a variety of novel resonators. It is the latter that are primarily of interest here.

Two common micromachining techniques to fabricate MEMS devices are called bulk and surface micromachining. Bulk micromachining [20] selectively removes amounts of material from a substrate using etching. The etching can be due to a liquid compound ("wet" etching) or due to a vapour or plasma ("dry" etching). Further, the etching can be equal in all directions ("isotropic" etching) or only in selective directions ("anisotropic" etching). A masking material is placed on the substrate in order to define where the etching is to take place, just like masking a wall before painting. Then, depending on the type of substrate, type of etchant, temperature, agitation and etching time, a variety of geometries can be produced in the substrate.

Surface micromachining [21] uses deposited thin films to build microelectromechanical structures on the surface of a substrate. Thin film materials originally designed for integrated circuits, such as polycrystalline silicon, silicon nitride, silicon dioxides and phosphosilicate glass for example, are sequentially deposited and then selectively removed to make three-dimensional structures on the surface of a substrate. These surface micromachined structures often have parts that are free to move. Thus, mechanical movement can now be a part of an electrical integrated circuit, such as in a sensor, actuator or a motor, or the movement can be used to assemble a structure, like a mirror, that is then used in the electrical circuit.

Micromachining has been used to make components at RF frequencies [22, 23], microwaves [24], millimetre waves [25] and even up to terahertz applications [26]. Although there have been some applications of micromachining on GaAs substrates [27], the vast majority have been on silicon substrates, which corresponds nicely with the recent interest in pushing silicon integrated circuits (both CMOS and $\mathrm{SiGe}$ ) to $100 \mathrm{GHz}$ and 

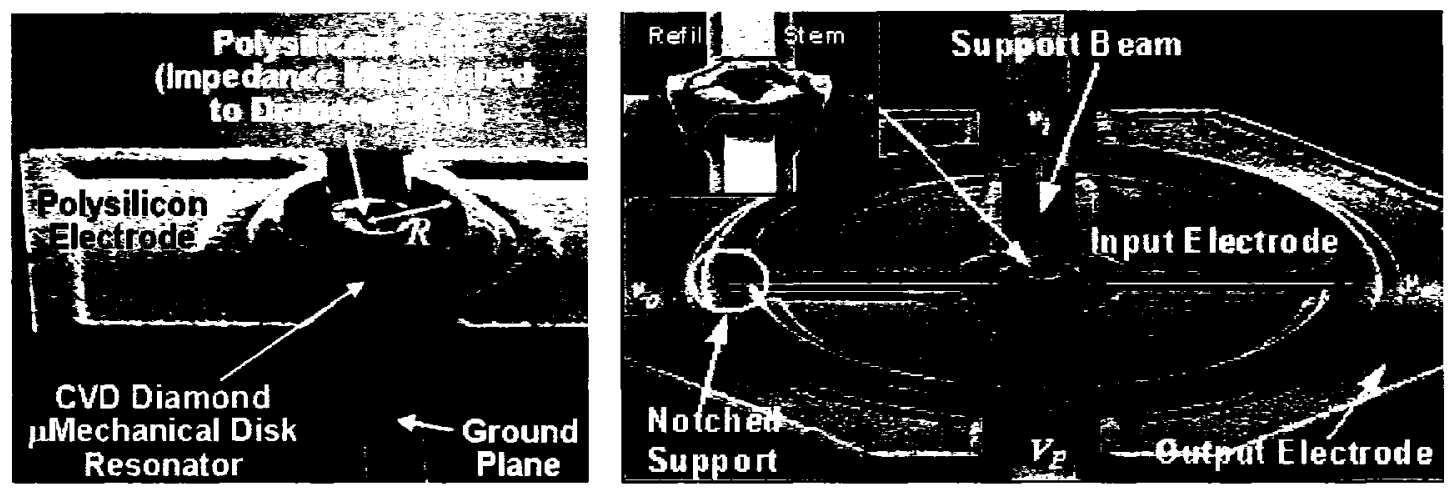

Figure 2.1: MEMS vibrating disk resonators with quality factors of 10,100 in air at $1.51 \mathrm{GHz}$ (left) [39] and 14,600 in a vacuum at $1.21 \mathrm{GHz}$ (right) [40].

beyond [28]-[30].

A variety of MEMS resonators, and oscillators stabilized by MEMS resonators, have appeared in the literature [31]. MEMS vibrating resonators have the highest quality factors because surface micromachining is used to create vibrating elements that are sensed electrically. The vibrating resonance remains stable over time since the quality factor depends almost entirely on the material properties of the vibrating element. Most vibrating MEMS resonators have been reported at frequencies below $1 \mathrm{GHz}$ [32]-[38] however in 2004 two were reported with quality factors exceeding 10,000 while resonating at $1.51 \mathrm{GHz}$ [39] and $1.2 \mathrm{GHz}$ [40]. Photographs of these vibrating MEMS are shown in Figure 2.1, but despite these encouraging results no millimetre wave vibrating MEMS have been reported. Frequency dependent loss mechanisms, such as impedance matching, power handling, noise, and temperature stability, may still impose upper frequency limits [41, 42]. Further, environmental factors, like atmospheric damping and moisture effects, lower the achievable quality factor.

At frequencies just above that of vibrating MEMS, micromachined inductors and capacitors have been reported with improved quality factors [43]-[56]. For micromachined inductors, the quality factor is improved by lowering the effect of the substrate losses by 


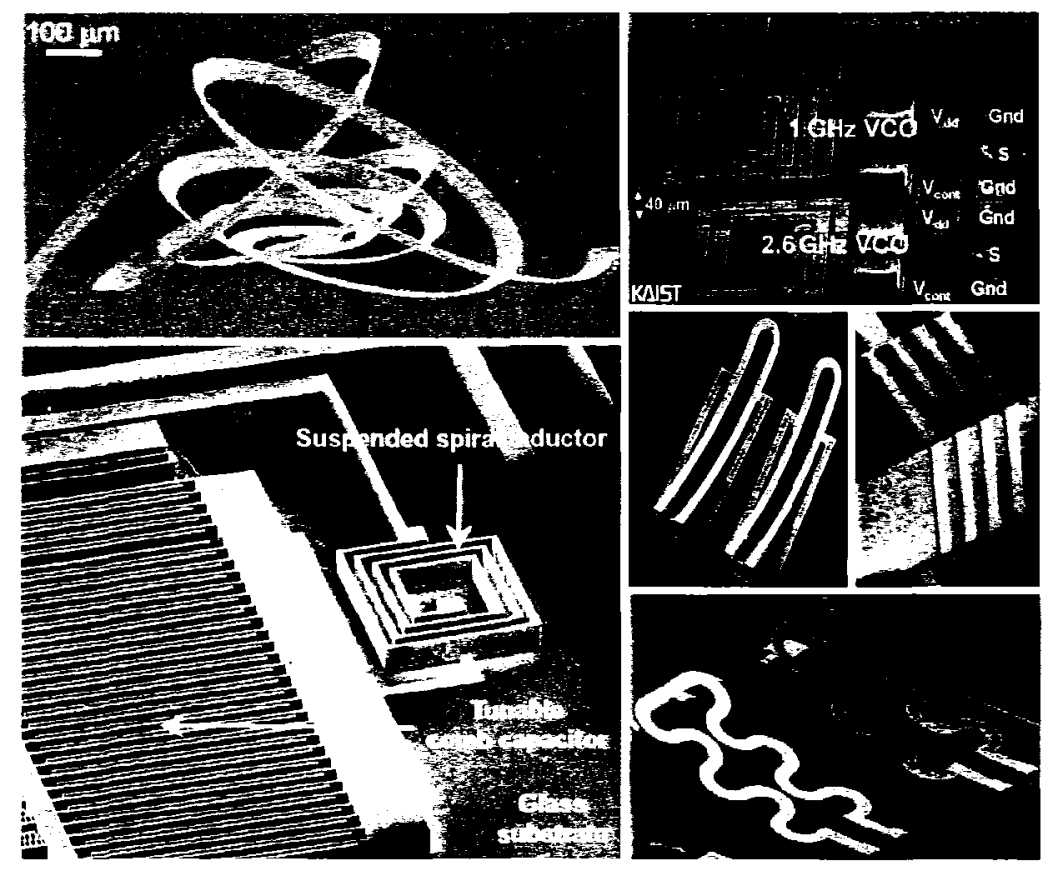

Figure 2.2: Examples of surface micromachined inductors $[46,53,55,56]$.

either removing portions of the substrate using bulk micromachining or using surface micromachining to suspend the conductors above the substrate. Examples of the latter technique are pictured in Figure 2.2, which can result in quality factors of up to 35 below $12 \mathrm{GHz}$ [51]-[53]. Higher quality factors of up to 100 have been reported below $20 \mathrm{GHz}$ [54], however, currently there are no published results at W-band.

Micromachined transmission line resonators on the other hand have been reported at millimetre waves. By micromachining the substrate and using a membrane supported transmission line, higher $Q$ resonator elements have been used for filters up to $117 \mathrm{GHz}$ [57]. Furthermore, an extension of this concept is to shield the transmission line using a metalized micromachined cavity. The conductors are surrounded by air as the dielectric and then enclosed by a cavity with walls that are ground, thereby reducing the loss and increasing the $Q$ [58]. Micromachined membrane stripline resonators, enclosed by micromachined cavities, have been reported with an unloaded $Q=386$ at 

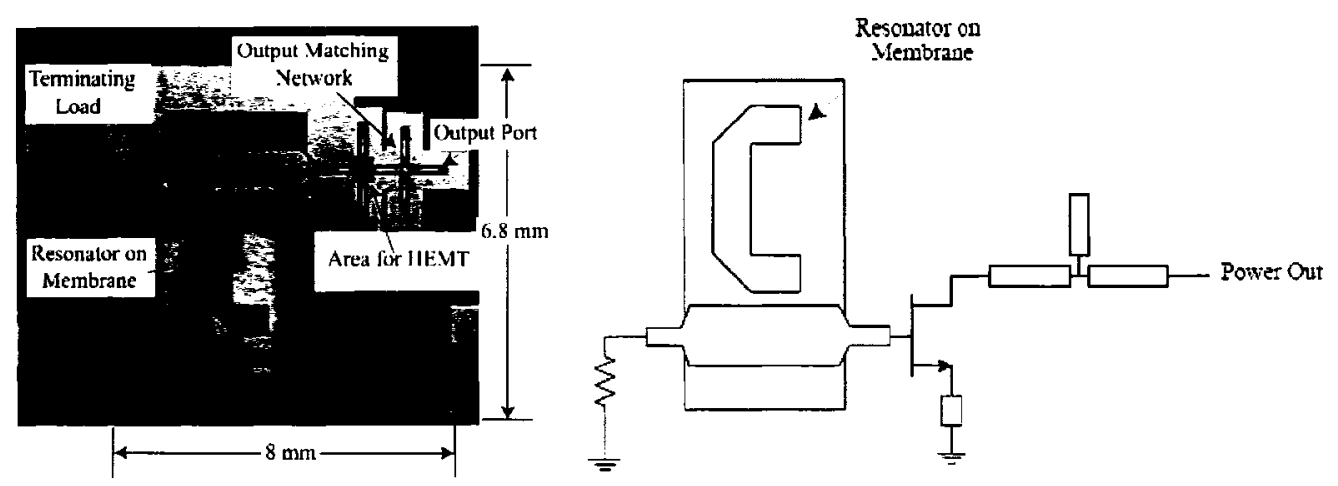

Figure 2.3: Micromachined membrane-supported microstrip oscillator [62, 63].
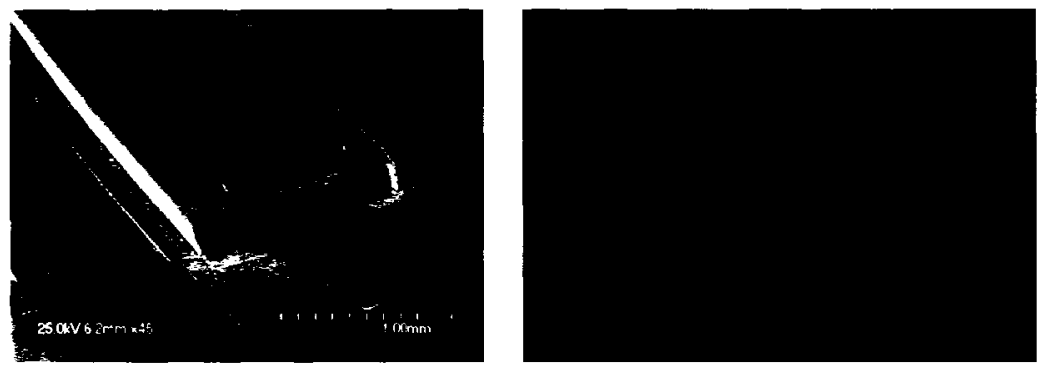

Figure 2.4: Non-planar micromachined transmission line resonators having $Q_{o}=153$ at $32 \mathrm{GHz}$ (left) [64] and $Q_{o}=200$ at $77 \mathrm{GHz}$ (right) [65].

$27 \mathrm{GHz}[59]$. There are also examples of membrane supported suspended microstrip resonators with unloaded $Q$ 's of about 400 at 29,37 and $60 \mathrm{GHz}$ [60,61]. Micromachined transmission line resonators also have been used to stabilize oscillators, such as shown in Figure 2.3, where a $28.65 \mathrm{GHz}$ oscillator was stabilized by a membrane supported, air dielectric transmission line resonator shielded by a micromachined cavity having an unloaded $Q_{o}=460$ and a loaded $Q_{l}=190[62,63]$.

Non-planar micromachined transmission line resonators have also been recently published such as those shown in Figure 2.4. A micromachined half-coaxial resonator has a measured unloaded quality factor of 153 at $32 \mathrm{GHz}$ [64] whereas a micromachined rectangular-coax resonator has a measured quality factor of 200 at $77 \mathrm{GHz}$ [65]. The latter example is of particular note since it resonates at W-band, however another type 


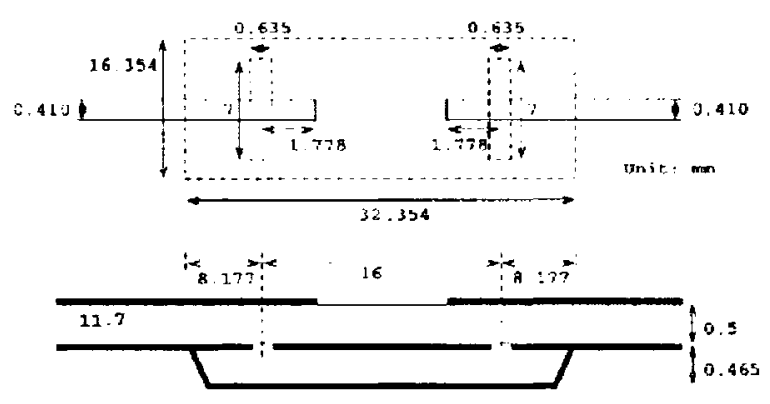

Figure 2.5: Micromachined cavity resonator [66].

of MEMS resonator, namely a micromachined cavity, is capable of even higher quality factors at this frequency.

Micromachined cavities, which are based on waveguide cavity resonators, are generally bulk micromachined from a suitable substrate. Waveguide cavity resonators have higher quality factors than LC-tanks or tranmission line resonators. This is also true for micromachined cavities, which have higher quality factors than both micromachined inductors and micromachined transmission line resonators. Following in the tradition of rectangular cavities, a micromachined "rectangular" cavity resonator was first reported in 1997 [66] and is shown in Figure 2.5. Using anisotropic bulk etching of a silicon wafer, a $450 \mu \mathrm{m}$ deep cavity was anisotropically etched and then metalized to a thickness of $2 \mu \mathrm{m}$. Another silicon wafer was also metalized on one side, except for two coupling slots, and then bonded to the first wafer using silver epoxy to create a sealed cavity. Two microstrip feed lines are patterned onto the non-metalized side of the second wafer for coupling through the slots. The cavity resonant frequency was $f_{o}=10.285 \mathrm{GHz}$ and the unloaded quality factor was $Q_{o}=506$. A rectangular waveguide cavity having the same dimensions as the micromachined cavity has an unloaded quality factor of $Q_{o}=526$. Therefore, the measured unloaded quality factor of the micromachined cavity is quite similar to a conventional waveguide cavity of the same dimensions. The loaded quality 

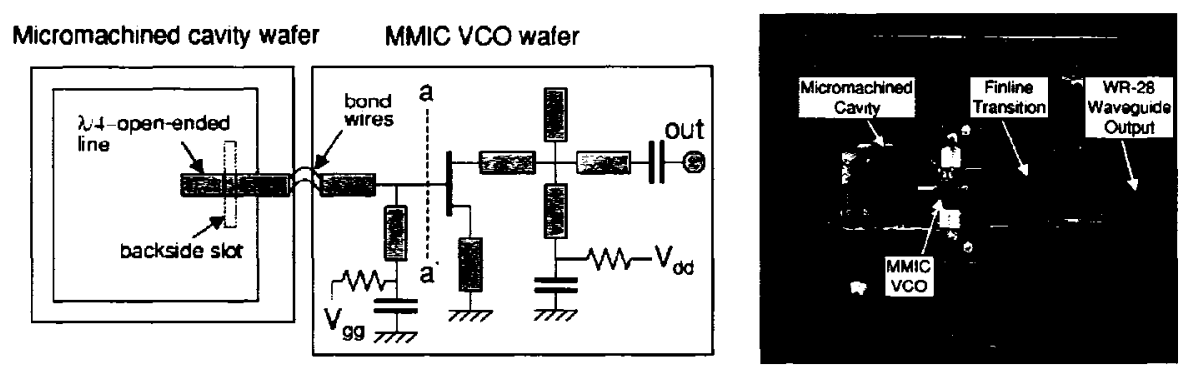

Figure 2.6: Micromachined cavity oscillator schematic (left) and picture (right) [69].

factor was only $Q_{l}=20$, however, hence the coupling mechanism was obviously not optimized.

The cavity [66] in Figure 2.5 has been used in a filter [67] and very similar cavities have been used in a diplexer [68] and a hybrid oscillator [69, 70]. The hybrid oscillator, shown in Figure 2.6, has an output frequency of $33.26 \mathrm{GHz}$ and the measured phase noise was $-85 \mathrm{dBc} / \mathrm{Hz}$ and $-113 \mathrm{dBc} / \mathrm{Hz}$ at $100 \mathrm{kHz}$ and $1 \mathrm{MHz}$ offsets respectively. The bottom half of its stabilizing cavity was micromachined from a silicon wafer, using anisotropic etching, to a depth of $400 \mu \mathrm{m}$ with lateral dimensions of $8.4 \mathrm{~mm} \times 5.8 \mathrm{~mm}$. The top half of the cavity was glass, with one side being used for the microstrip feed and the other side being the ground for the microstrip and the top wall of the cavity. The microstrip feed couples to the cavity through a slot in the ground plane and a quarter-wave stub ensures a short circuit at the slot for maximum current and hence maximum magnetic coupling. The feed and walls were metalized with gold to a thickness of $5 \mu \mathrm{m}$. The two cavity halves were aligned using an infrared aligner and bonded together with silver epoxy. By itself, this micromachined cavity resonates at $30.14 \mathrm{GHz}$ ( $\mathrm{TE}_{101}$ mode) with a $Q_{l}=120[71]$.

The single-wafer, anisotropically etched cavity geometry has been shown to operate at higher frequencies, such as $27 \mathrm{GHz}$ [72] and $39 \mathrm{GHz}$ [73], with the latter example pictured in Figure 2.7. There have also been reports on reducing the size of the geometry by 


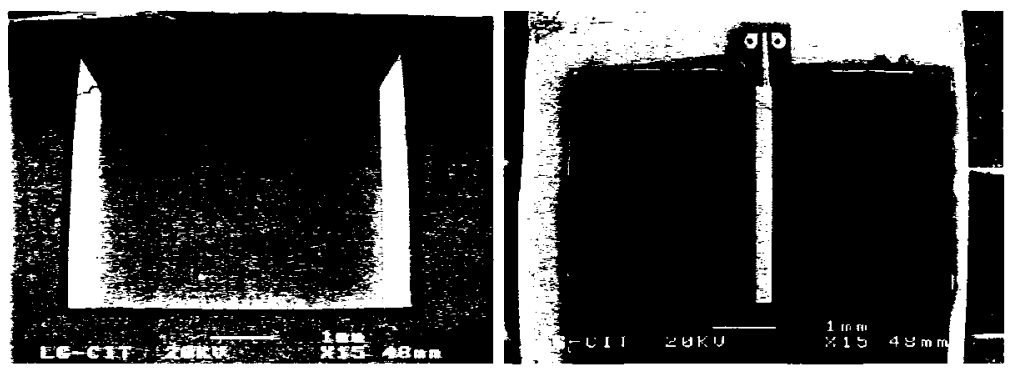

Figure 2.7: Single-wafer micromachined cavity resonator [73]: anisotropically etched cavity without a feed (left) and with a feed bonded to seal the cavity (right).

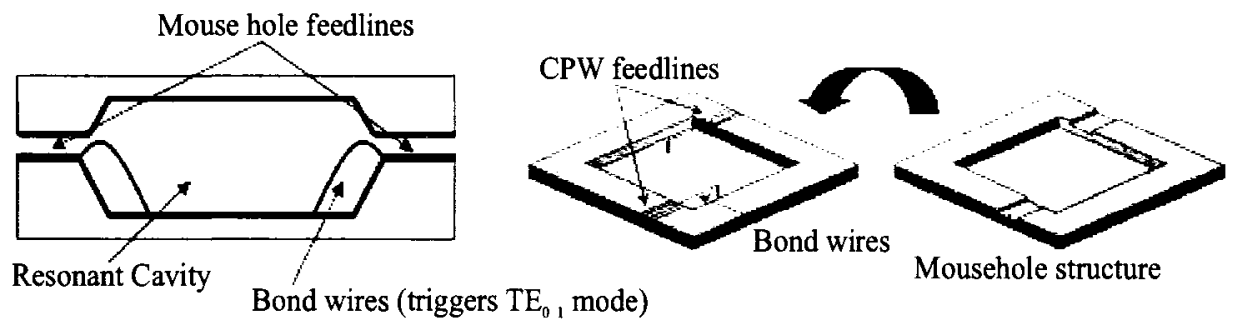

Figure 2.8: Schematic of a two-wafer micromachined cavity [61].

either filling the micromachined cavity with dielectric [74,75], or by using micromachined posts for evanescent mode operation [76]. In the former example, square-base, $5.7 \mathrm{GHz}$ cavities were micromachined and then filled with barium strontium titanate (BST) having $\epsilon_{r}=70$. As would be expected, the size of the cavities decreased by a factor of $\sqrt{\epsilon_{r}}$, however, the quality factor also decreased according to the extra loss introduced by the dielectric.

For micromachined cavities, it has been reported that when the metalization is at least 3 skin depths, the cavity energy will not penetrate through the metal to the silicon body (and eventually radiate into free space) [77], which is similar to waveguide cavities [78, 79]. Thus, attempts to improve the quality factor of anisotropically etched cavities have focused on increasing the depth of the cavities. One approach was to use two wafers that are etched and joined to form a cavity, shown in Figure 2.8 [61]. By etching two wafers 


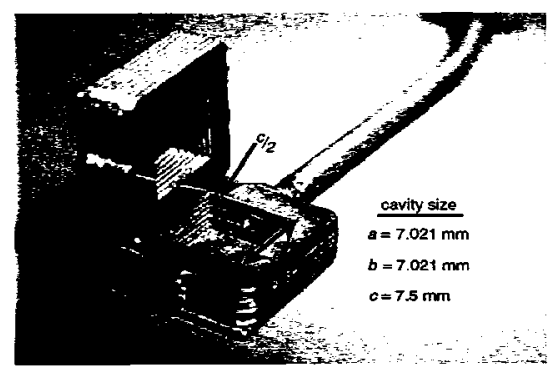

Figure 2.9: Stacked-wafer micromachined cavity resonator [83].

instead of one a cavity twice as thick results, and this improves the quality factor of the resonator since the $Q$ is strongly related to the thickness in a rectangular resonator. The two silicon wafers were etched to a depth of $450 \mu \mathrm{m}$. The bottom wafer is high resistivity silicon $(2000 \Omega \mathrm{cm})$ with a CPW line defined on the bottom wafer at the edge of the cavity. A bond wire is then placed from the end of the CPW line to the bottom of the cavity to act as a loop exciting the dominant mode at resonance. The amount of coupling is controlled by the length and number of bond wires used. The top wafer is low resistivity silicon $(5-7 \Omega \mathrm{cm})$ and is identical to the bottom wafer except that a groove is etched above the CPW line so that the CPW is not shorted when the two wafers are bonded together with silver epoxy. The feed loops are placed in the corners of the cavity, where the current on the side walls is minimum, to minimize the disturbance to the cavity. The cavity was designed for $24 \mathrm{GHz}$, which corresponds to lateral dimensions of $8.84 \mathrm{~mm} \times 8.84 \mathrm{~mm}$, and the measured performance is $23.97 \mathrm{GHz}$ with $Q_{o}=1117$.

Another method used to increase the depth of anisotropically etched cavities is to etch all the way through multiple cavities and then stack them together to form a much larger cavity [80]-[83]. An extreme example is shown in Figure 2.9 where the cavity depth is $15 \mathrm{~mm}$ [83], however, this is much too large to be a fair comparison to other published micromachined cavities whose depths are less than $1.5 \mathrm{~mm}$.

Another type of micromachined cavity that has been published takes a different 


\begin{tabular}{|c|c|c|c|}
\hline Frequency, GHz & Unloaded Q & Loaded Q & Reference \\
\hline \hline 5.656 & 636 & - & {$[75]$} \\
5.915 & 154 & - & {$[75]$} \\
10.285 & 506 & 20 & {$[66]$} \\
11.84 & 303 & 253 & {$[80]$} \\
14 & 310 & - & {$[76]$} \\
20.45 & 890 & 786 & {$[68]$} \\
22 & - & 70 & {$[84]$} \\
22.5 & 366 & 126 & {$[85]$} \\
23.97 & 1117 & - & {$[61]$} \\
26.8 & 125 & - & {$[72]$} \\
27.88 & 1465 & - & {$[67]$} \\
30.14 & - & 120 & {$[71]$} \\
32.5 & - & 50 & {$[84]$} \\
39 & 107 & 44.3 & {$[73]$} \\
60.03 & 1100 & - & {$[86]$} \\
\hline
\end{tabular}

Table 2.1: Measured performance of published micromachined cavity resonators with depth less than $1.5 \mathrm{~mm}$ ('-' means quantity not given in publication).

approach, where instead of anisotropically etching the cavity itself, a wall of vias are anisotropically etched to form the cavity walls $[84,85]$. A published combination of a three-layer cavity (where two dry-etched cavities sandwich one via-wall etched cavity) has a measured unloaded quality factor of 1100 at $60.03 \mathrm{GHz}$ thus confirming the suitability of micromachined cavities at millimetre wave frequencies [86].

The measured performance of all of the micromachined cavities described in this section are listed in Table 2.1 .

\subsection{The Perfectly Hemispherical Resonator}

A resonator is a source-free region, enclosed by a boundary, that can store electromagnetic energy at certain resonant frequencies. This stored energy decays over time due to losses in the resonator and the quality factor is a measure of this capability to store energy 
in the presence of loss. Together, each resonant frequency and associated quality factor correspond to a resonant mode.

Relating the resonant frequency and quality factor to the physical parameters of an electromagnetic resonator is a field boundary value problem. This may be difficult if the resonator shape and coupling mechanism is complex. Usually, the resonator geometry is simplified and the coupling ignored by closing the structure in order to theoretically solve the for the "unloaded" resonant frequency and "unloaded" quality factor. Then the coupling mechanism may be added to determine the "loaded" resonant frequency and the "loaded" quality factor. In either case, when the resonator is bounded by conductors, the boundary conditions of normal electric field and tangential magnetic field are used to solve Maxwell's equations for the resonator structure, assuming no losses. The solutions to Maxwell's equations are the resonant modes and they occur at discrete resonant frequencies. Each mode has an associated field configuration that is useful for coupling mechanism design. Next, losses, which are a function of the materials of the resonator, are included and the quality factors for each of the modes are found. Thus, the resonant frequencies and quality factors of each mode are functions of the physical structure and the materials of the resonator.

The unloaded and loaded resonant frequencies differ due to the reactive components of the coupling mechanism. The difference between the unloaded and loaded quality factors is more subtle. Considering that the quality factor is the ratio of the stored energy to the lost energy, and that energy is only stored in the resonator, then the difference between the unloaded and loaded quality factors must be where the energy is lost in the coupled resonator. Indeed, the unloaded quality factor is the ratio of energy stored in the cavity to the energy lost in the cavity. Another quantity, the external quality factor, is the ratio of the energy stored in the cavity to the energy lost in the system outside the cavity, since in order to be useful the resonator must be connected to something. The loaded 
quality factor accounts for both internal and external losses and is therefore the most important parameter when the resonator is used as a circuit element.

The amount of energy coupling to and from a resonator is also an important parameter. A coupling coefficient is used as this measure, and the coupling coefficient is defined as the ratio of the unloaded quality factor to the external quality factor. If the coupling coefficient is less than one the resonator is said to be "undercoupled" and only a small amount of energy enters and leaves the resonator. In this case the coupling is not seriously degrading the energy storage capability of the resonator. Alternatively, if the coupling coefficient is greater than one, then the resonator is said to be "overcoupled", and the coupling is degrading the energy storage of the resonator. A coupling coefficient of exactly one is said to be "critical coupling" since this is when the resonator is matched and the resonator is loosing about half the maximum possible energy it can store to the outside system. Of course, if one is designing a filter, matching at critical coupling may be desirable, however, for an oscillator design, undercoupling for maximum energy storage (and hence high loaded quality factor) is best to stabilize the oscillator.

Although waveguide cavities today are commonly rectangular or cylindrical, one of the earliest cavity resonators was a spherical cavity published in 1908 [87] in German and in 1938 in English [16]. Basic shapes, such as a square, cylinder and sphere, were compared to determine the most efficient shape in terms of quality factor but since not all of the shapes had been fully analyzed at that time, it was not certain which was the most efficient. It was guessed, however, that a sphere or a long cylinder would have the best quality factor. Despite this prediction, square and cylindrical cavities were heavily researched in the years that followed, and continue to be today. Fields have been calculated for a general cavity [88] and charts exist that plot a large number of the resonant frequencies and mode configurations for square and cylindrical cavities [89, 90]. Spherical cavities seemed to have dropped out of favour. There are some published 


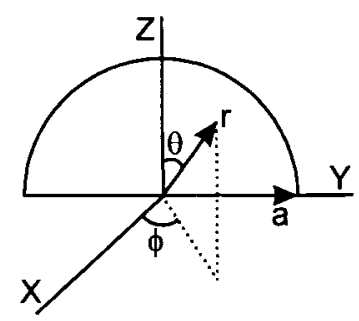

Figure 2.10: Spherical coordinate system for a perfectly hemispherical cavity.

examples of spherical cavities [91]-[97] and even the excitation of arbritrary modes has been examined [98], but the dominant form of cavities have been cylindrical (although ironically mode separation in cylindrical cavities is a problem that can be solved by making them more spherical, such as rounding the ends [99]). Nevertheless, waveguide spherical resonator theory is well established [17] and perfectly suited to be applied to an isotropically etched cavity.

The first five resonant modes for the hemispherical cavity in Figure 2.10 are plotted in Figure 2.11. When the lowest order resonance, known as the fundamental mode, is at $77 \mathrm{GHz}$, then the next resonances are at $108 \mathrm{GHz}$ and $126 \mathrm{GHz}$, both of which include two degenerate modes rotated $90^{\circ}$ with respect to one another. The resonant frequencies can be calculated for each mode, depending on the type (i.e. a $T E$ or $T M$ mode), using [17]:

$$
\begin{aligned}
& \left(f_{r}\right)_{m n p}^{T E}=\frac{u_{n p}}{2 \pi a \sqrt{\epsilon_{o} \mu_{o}}} \\
& \left(f_{r}\right)_{m n p}^{T M}=\frac{u_{n p}^{\prime}}{2 \pi a \sqrt{\epsilon_{o} \mu_{o}}}
\end{aligned}
$$

where the radius, $a$, is defined in Figure 2.10. These resonant frequencies are proportional to either $u_{n p}$ or $u_{n p}^{\prime}$, which are the ordered zeros of the spherical Bessel function and the derivative of the spherical Bessel function, respectively. Since the ordered zeros are, in ascending order, $u_{11}^{\prime}=2.744, u_{21}^{\prime}=3.870, u_{11}=4.493, u_{31}^{\prime}=4.973, u_{21}=5.763$ and 


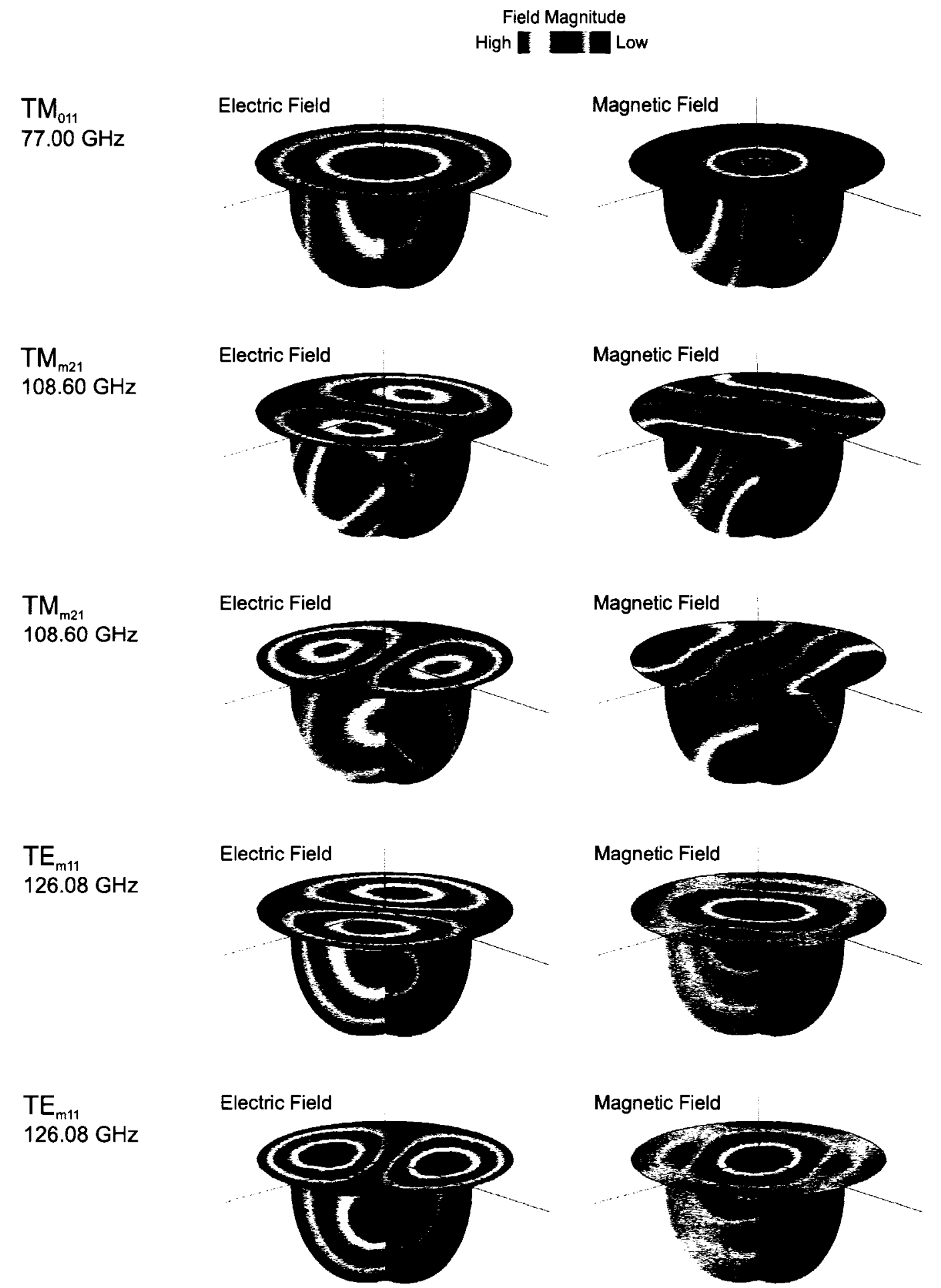

Figure 2.11: Five lowest order mode patterns for a perfectly hemispherical resonator. 
so on, then the modes in terms of ascending resonant frequencies are $T M_{m, 1,1}, T M_{m, 2,1}$, $T E_{m, 1,1}, T M_{m, 3,1}, T E_{m, 2,1}$ and so on. Since the resonant frequencies are independent of $m$, there are degenerate modes as seen in Figure 2.11.

The lowest order mode of a perfectly hemispherical cavity is the same as for a perfectly spherical cavity, except that there is no degeneracy. Two of the three degenerate fundamental modes in a spherical cavity cannot exist in a hemispherical cavity since these two have tangential electrical fields on the $x y$ plane which cannot exist due to the metal wall boundary condition. Therefore, the fundamental mode of a perfectly hemispherical cavity is a single $T M_{011}$ mode with a resonant frequency given by equation 2.2 and it results in the smallest cavity size for a desired frequency. For this reason, the fundamental mode is often chosen as the operating mode for cavity resonators.

The field components of the $T M_{011}$ mode in a perfectly hemispherical cavity are:

$$
\begin{aligned}
& E_{r}=\frac{2 \cos \theta}{u_{11}^{\prime} r^{3}}\left[a \sin \left(u_{11}^{\prime} \frac{r}{a}\right)-u_{11}^{\prime} r \cos \left(u_{11}^{\prime} \frac{r}{a}\right)\right] \\
& E_{\theta}=\frac{\sin \theta}{u_{11}^{\prime} a r^{3}}\left[a^{2} \sin \left(u_{11}^{\prime} \frac{r}{a}\right)-u_{11}^{\prime} a r \cos \left(u_{11}^{\prime} \frac{r}{a}\right)-u_{11}^{\prime 2} r^{2} \sin \left(u_{11}^{\prime} \frac{r}{a}\right)\right] \\
& E_{\phi}=0 \\
& H_{r}=0 \\
& H_{\theta}=0 \\
& H_{\phi}=\frac{\sin \theta}{u_{11}^{\prime} r^{2}}\left[a \sin \left(u_{11}^{\prime} \frac{r}{a}\right)-u_{11}^{\prime} r \cos \left(u_{11}^{\prime} \frac{r}{a}\right)\right]
\end{aligned}
$$

Using equations 2.3 to 2.8 , the calculated magnitude of the $\vec{E}$ and $\vec{H}$ fields of the fundamental $T M_{011}$ mode are shown in Figure 2.12 for the $x y, y z$ and $x z$ planes of Figure 2.10.

The unloaded quality factor, for the fundamental $T M_{011}$ mode of the perfectly hemispherical cavity, is [17]

$$
Q_{o}=0.573 \frac{\eta}{\mathcal{R}}
$$


Calculated fields of the fundamental mode of a perfectly hemispherical cavity High field magnitude

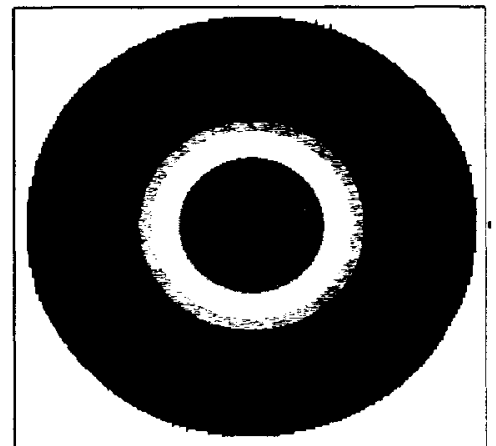

Electric field, $x y$-plane

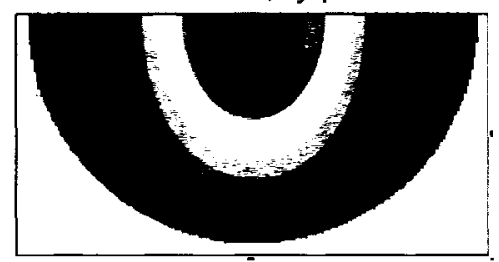

Electric field, $\mathbf{x z}$ - \& yz-planes

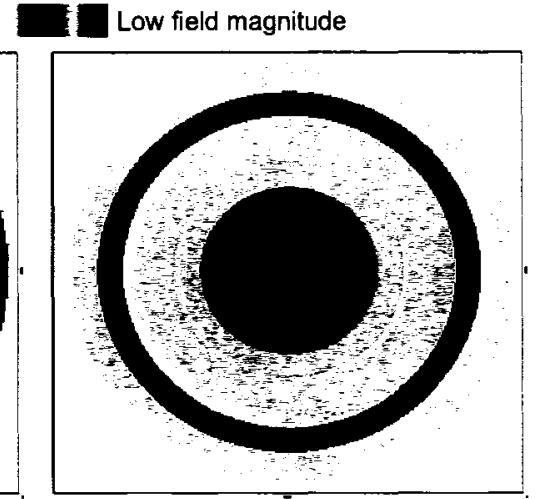

Magnetic field, $x y$-plane

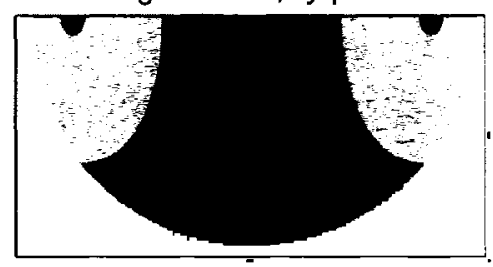

Magnetic field, $x z-\&$ yz-planes

Figure 2.12: Theoretical $|\vec{E}|$ and $|\vec{H}|$ for a perfectly hemispherical cavity calculated using equations 2.3 to 2.8 for the $T M_{011}$ mode.

where $\eta=377 \Omega$ is the intrinsic impedance of free space and $\mathcal{R}$ is the surface resistance of the conductor, which for good conductors is [17]

$$
\mathcal{R}=\sqrt{\frac{2 \pi f \mu_{o}}{2 \sigma}}
$$

where $\sigma$ is the conductivity of the metal. Thus increasing frequency and decreasing conductivity increases the surface resistance and lowers the unloaded quality factor of the $T M_{011}$ mode in the cavity. When the walls are gold and $f=77 \mathrm{GHz}$ then the ideal unloaded quality factor of the $T M_{011}$ mode is $Q_{o}=2506$.

The $T M_{011}$ mode can be excited by an aperture in the $x y$-plane with a microstrip line above (the $x y$-plane being both the cavity wall and the microstrip ground plane). In this scenario, the microstrip excites a magnetic field vector in the aperture with the 
$\mathrm{TM}_{011}$ Electric Field
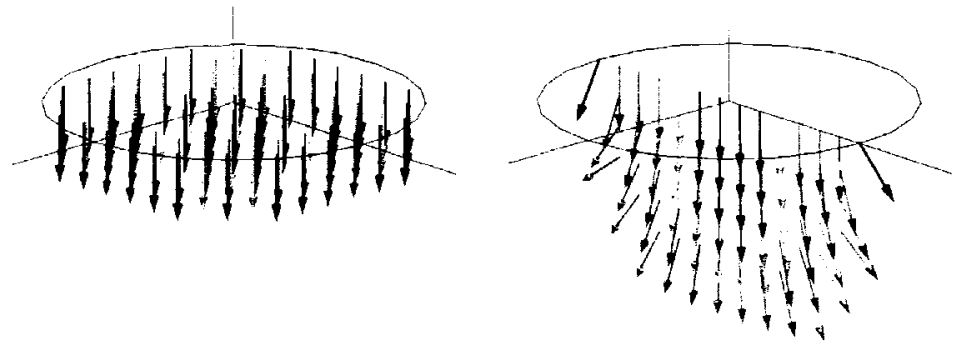

$\mathrm{TM}_{011}$ Magnetic Field

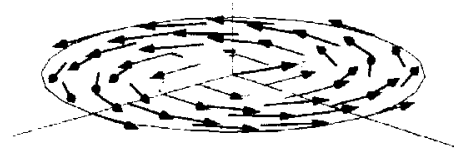

High $\square$ Low

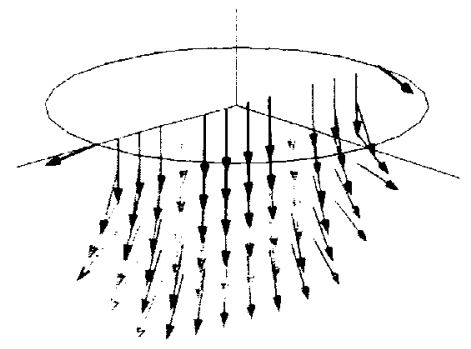

High 1 Low
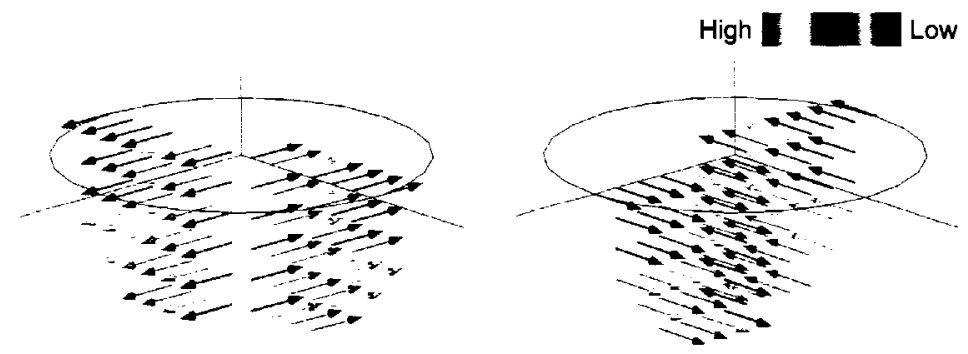

Figure 2.13: Fundamental mode pattern for a perfectly hemispherical resonator.

same orientation as the $x y$-plane magnetic field vector shown in Figure 2.13, which is the vector $T M_{011}$ mode pattern. This excites the $T M_{011}$ mode in the cavity.

The fields in the vicinity of the aperture may be approximated by the combination of the original fields before the aperture is opened and the fields of electric and magnetic dipoles located at the centre of the aperture [100]-[102]. The electric dipole is located perpendicular to the plane of the aperture and the magnetic dipole is located in the plane of the aperture. The strengths of these dipoles, known as dipole moments, are related to the original fields by constants of proportionality called electric and magnetic polarizabilities. The electric polarizability is a scalar constant, $P$, and the dipole and the field are parallel. The magnetic polarizability is a dyadic quantity and is a function of the aperture geometry since the directions of the magnetic dipole and the exciting field are not necessarily the same. The principal axes of the aperture can be chosen to correspond to the axes of symmetry of the aperture, if they exist, and then the magnetic 
polarizability is determined by two scalar constants, $M_{1}$ and $M_{2}$. This approximation of aperture coupling assumes that the aperture is far from any perturbations in the wall and is also small compared to the wavelength. Furthermore, the aperture wall is assumed to be infinitely thin and a perfect conductor. Collectively, these assumptions are known as the "small aperture" assumption.

\subsection{Isotropic Wet Etching}

The micromachining technique used to fabricate the hemispherical cavity resonators is known as "wet etching". Wet etching has been traditionally used for cleaning and polishing materials, however it has recently been applied to micromachining substrates [103][111]. There are three basic steps to wet etch a substrate: first, transport the reactant to the surface of the substrate, second, the surface reaction, and third, transport the reactant product away from the surface of the substrate. If the first and the third steps limit the etch rate then the etching is said to be diffusion limited. If the second step limits the etch rate then the etch is said to be reaction rate limited. Stirring may increase the etching if it is diffusion limited as this enhances the reactant product transport. Diffusion limited etching is also fairly insensitive to temperature. For a reaction rate limited situation, then the etch rate is dependent on the material and temperature.

There are two types of wet etching: isotropic and anisotropic. Isotropic etching etches all crystallographic directions at the same rate. These were some of the first etchants introduced and are usually acidic. Often, isotropic etchants are diffusion limited. Anisotropic etchants etch different directions at different rates, thus the resulting etch is highly dependent on crystal orientation. Anisotropic etchants are usually alkaline and are often reaction rate limited.

Many of the existing micromachined cavities are anisotropically etched, that is rect- 


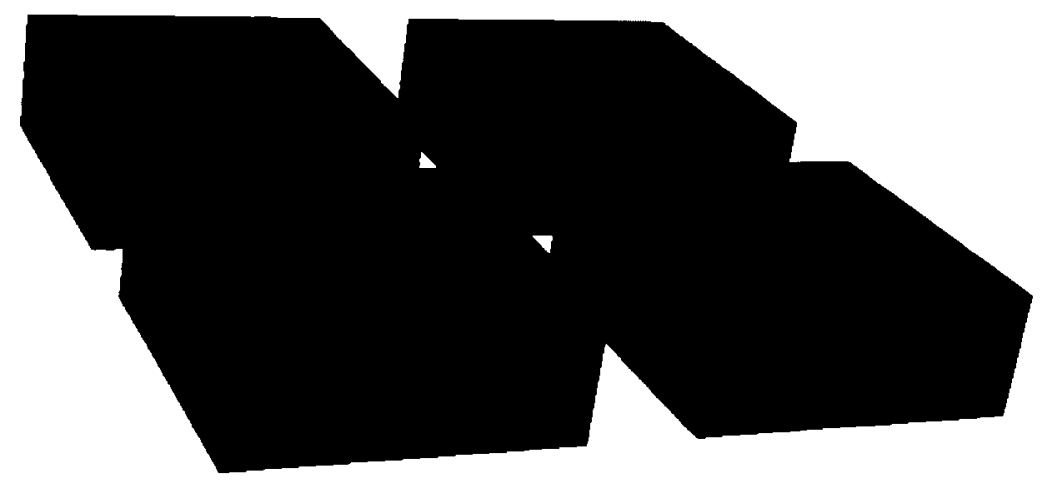

Figure 2.14: Cavity geometries micromachined in a substrate: isotropically etched (left) and anisotropically etched (right). Substrates shown transparent in foreground to highlight the etched geometry.
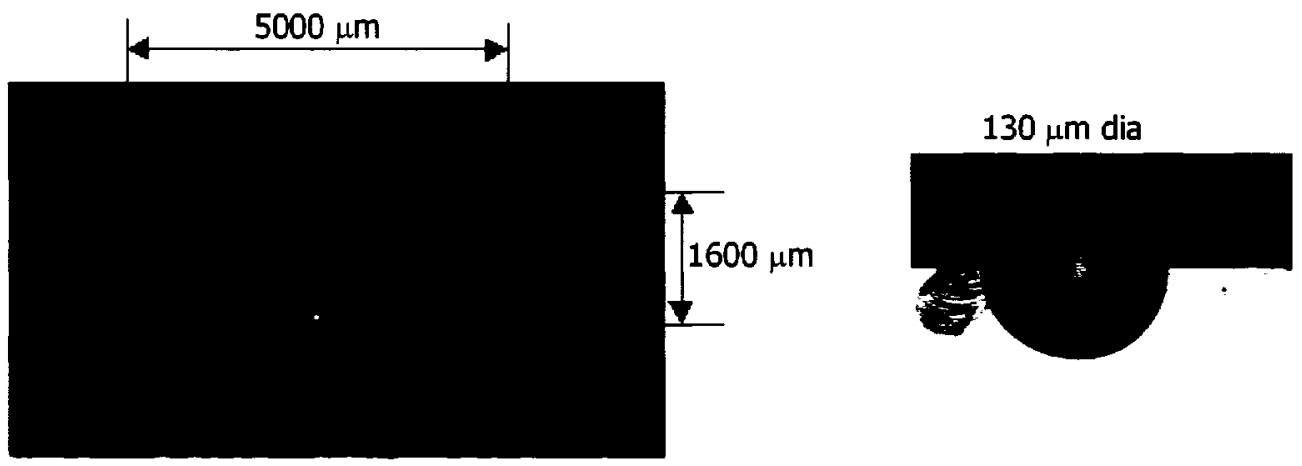

Figure 2.15: Cross-sectional views of existing micromachined hemispherical structures for acoustic microphones (left) [112] and thermofusion nuclear pellets (right) [113].

angularly shaped with sloping side walls as shown in Figure 2.14 on the right. Since isotropic etching results in all directions being etched at approximately the same rate, isotropic etching results in an approximately hemispherical shape suitable for micromachined cavities, as shown in Figure 2.14 on the left. Some previously reported examples of isotropically etched hemispheres are for acoustic microphones [112] and for thermofusion nuclear pellets [113], both shown in Figure 2.15, and this provides a basis from which to develop a custom micromachining procedure for hemispherical cavity resonators.

There are a variety of etchant "systems" to isotropically etch silicon $[105,106]$, but 
perhaps the most common etchant is a combination of nitric acid $\left(\mathrm{HNO}_{3}\right)$ and hydrofluoric acid (HF) with either water $\left(\mathrm{H}_{2} \mathrm{O}\right)$ or acetic acid $\left(\mathrm{CH}_{3} \mathrm{COOH}\right)$ as a diluent. This system has come to be known as the HNA System [107]-[110]. Each acid solution in the HNA System is generally available commercially as a certain percentage of a water solution, such as $49 \%$ hydrofluoric acid with $51 \%$ water (called " $49 \%$ hydrofluoric acid" for short). Three commercially available acid solutions commonly used in HNA are $49 \%$ hydrofluoric, $70 \%$ nitric and $99 \%$ acetic acids and there are a variety of etch formulations for the HNA system depending on the ratio of these hydrofluoric, nitric and acetic acids. This ratio is specified by "parts by volume" of each acid solution such as $1: 1: 2$. The ratio of each of these acids affects both the etch rate and the surface finish [110, 114].

For a given acid ratio, the etch rate is constant if the etchant is replenished, however, etching rate is not constant if the etchant is not replenished. The temperature of the etch may also affect the rate (depending on the specific formulation of the acids), and generally increasing the temperature increases the etch rate [109].

Agitation may affect the sphericity of a etched cavity. HNA etching is a diffusion limited process, so agitating the etchant will alter the process of transporting reactants to the silicon surface and transporting by-products away. The amount of impact agitation has, however, is debatable. Some sources claim that agitation may increase the sphericity of the etched surface $[19,113]$ whereas others have reported an acceptable level of sphericity without any agitation $[112,115]$. 


\section{Chapter 3}

\section{Design of Micromachined}

\section{Hemispherical Cavity Resonators}

This chapter presents a novel method to design micromachined hemispherical cavity resonators. Equations have been developed for the resonant frequency, unloaded and loaded quality factors of an oblate hemispherical micromachined cavity that is aperture coupled to microstrip. This chapter also includes simulations that verify the calculated values of the resonant frequency, unloaded and loaded quality factor.

\subsection{Resonant Frequency}

The fundamental resonant frequency for a perfectly hemispherical cavity resonator is well known and was given in equation 2.2. In theory, isotropic etching a cavity results in a perfect hemisphere with resonant frequencies predicted by equation 2.2 . In practice, however, this is not the case. As will be described in detail in Chapter 4, the actual, isotropically etched cavities are not perfectly hemispherical but are, in fact, oblate hemispheroids. 
Nevertheless, equation 2.2 for a perfect hemisphere provides a basis to start the analysis of the actual micromachined cavity geometry. Since the desired operating frequency at $\mathrm{W}$-band, $77 \mathrm{GHz}$, is known the necessary cavity radius for a $T M_{011}$ perfectly hemispherical cavity is found by re-arranging equation 2.2 to become

$$
a=\frac{u_{11}^{\prime}}{2 \pi f_{o} \sqrt{\epsilon_{o} \mu_{o}}}
$$

where the operating frequency is $f_{o}=\left(f_{r}\right)_{011}^{T M}, \sqrt{\epsilon \mu} \approx \sqrt{\epsilon_{o} \mu_{o}}$ since the cavity contains only air, and $u_{n p}^{\prime}=u_{11}^{\prime}=2.744$. The $T M_{011}$ mode was chosen because, as stated in Section 2.2 , the $T M_{011}$ mode results in the smallest cavity size at $77 \mathrm{GHz}$.

For the desired operating frequency of $f=77 \mathrm{GHz}$, equation 3.1 gives a required design radius of $a=1.7004 \mathrm{~mm}$. Thus, for a perfectly hemispherical cavity resonator with this radius the resonant modes are, in ascending order: $T M_{011} \rightarrow 77.002 \mathrm{GHz}$, $T M_{m 21} \rightarrow 108.600 \mathrm{GHz}, T E_{m 11} \rightarrow 126.083 \mathrm{GHz}, T M_{m 31} \rightarrow 139.553 \mathrm{GHz}, T E_{m 21} \rightarrow$ $161.722 \mathrm{GHz} \ldots$ and so on.

In Chapter 4, however, it is proven that when cavities are actually micromachined, and then soldered to an alumina substrate to seal the cavity, they are not perfectly hemispherical. Compared to the perfect hemisphere with radius $a$, the actual micromachined and assembled cavities are oblate hemispheres with lateral radius $a$ and a smaller average theoretical depth, $c$, introduced in Section 4.1. The actual cavities also have an additional cylindrical volume due to the thickness, $h$, of the solder as described in Section 4.4. Hence, equation 2.2 does not give the resonant frequency of the actual cavity geometry but it can be used with perturbation techniques [17] to calculate the actual resonant frequency of the fully-assembled micromachined cavity. The actual cavity is equivalent to a perfect hemisphere that has been perturbed twice, first by reducing the depth and second by adding the solder thickness. The change in resonant frequency due to these 


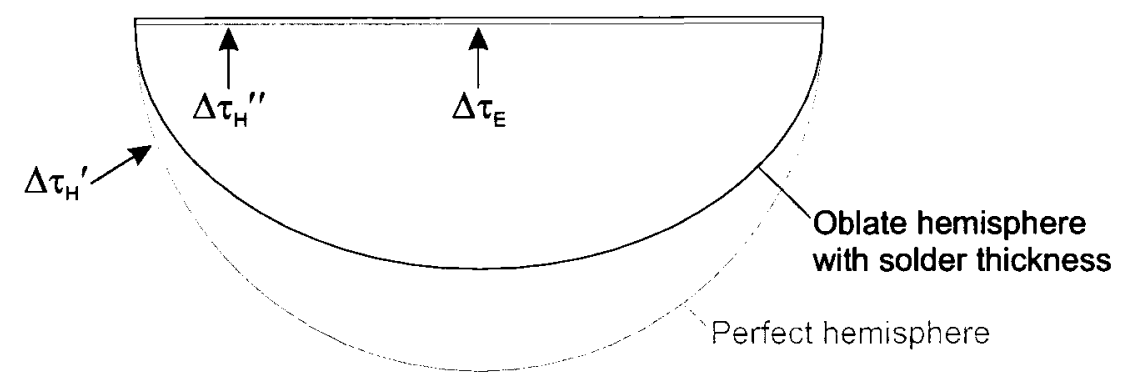

Figure 3.1: Perturbations of a perfect hemisphere resulting in an oblate hemisphere with solder thickness.

perturbations is roughly proportional to the volume of the perturbations relative to the original volume of the perfect hemisphere, assuming the perturbations are small.

Perturbations in the regions of maximum field strength affect the resonant frequency the most and the amount of change depends on whether the maximum is an $E$ or an $H$ field. These regions of maximum field for the perfect hemisphere can be easily seen in the field plots of Figure 2.12. The maximum $E$ field in the $x y$ plane occurs from approximately $0<r \leqslant 0.7 \mathrm{~mm}$ whereas the maximum $H$ field occurs from approximately $0.7<r \leqslant 1.7 \mathrm{~mm}$ in the $x y$ plane and from $0 \leqslant \theta \leqslant \frac{\pi}{6}$ for all $\phi$ (shown as $x z$ and $y z$ planes in Figure 2.12). In the region of maximum $E$ field, there is an outward perturbation of the top wall added by the solder layer. In the region of maximum $H$ field there are two perturbations: an inward perturbation of the curved wall (oblate hemisphere) and an outward perturbation of the top wall (solder layer). The remainder of the curved wall is also perturbed for $\theta>\frac{\pi}{6}$ by the oblate hemisphere but as shown in the $x z$ and $y z$ planes in Figure 2.12, this perturbation occurs at a much lower $H$ field and its change to the resonant frequency is assumed small enough to be ignored.

Figure 3.1 shows these three perturbations: $\Delta \tau_{E}$ is the volume of the outward perturbation of the top wall in the region of high $E$ field, $\Delta \tau_{H}^{\prime}$ is volume of the inward perturbation of the curved wall in the region of high $H$ and $\Delta \tau_{H}^{\prime \prime}$ is the perturbed volume of the outward perturbation of the top wall in the region of high $H$. If $\tau_{o}$ is the volume 
of the unperturbed perfect hemisphere with an unperturbed resonant frequency $f_{o}$, then the resonant frequency of the actual oblate hemisphere with solder using perturbation techniques is

$$
f \approx f_{o}+\left(0.68 \frac{\Delta \tau_{H}^{\prime}}{\tau_{o}}-0.68 \frac{\Delta \tau_{H}^{\prime \prime}}{\tau_{o}}+2.02 \frac{\Delta \tau_{E}}{\tau_{o}}\right) f_{o}
$$

where $f_{o}=\frac{u_{1}^{\prime}}{2 \pi a \sqrt{\epsilon_{o} \mu_{o}}}$ (equation 2.2) and the volumes are

$$
\begin{aligned}
\tau_{o} & =\frac{2}{3} \pi a^{3} \\
\Delta \tau_{H}^{\prime} & =\int_{0}^{2 \pi} \int_{0}^{\frac{\pi}{6}} \int_{a \sqrt{\frac{1-e^{2}}{1-e^{2} \cos ^{2}(\theta)}}}^{a} r^{2} \sin (\theta) \mathrm{d} r \mathrm{~d} \theta \mathrm{d} \phi \\
& =\frac{2 \pi a}{3}\left(a^{2}-c^{2}+c^{3} \sqrt{\frac{3}{a^{2}+3 c^{2}}}-\frac{a^{2} \sqrt{3}}{2}\right) \\
\Delta \tau_{H}^{\prime \prime} & =\pi h\left(a^{2}-0.007^{2}\right) \\
\Delta \tau_{E} & =0.007^{2} \pi h
\end{aligned}
$$

The eccentricity, $e$, is $e=\sqrt{1-\frac{c^{2}}{a^{2}}}$, where $a$ is the lateral radius, $c$ is the depth and $h$ is the solder thickness.

Nominally $a=1.7 \mathrm{~mm}$ (p. 59), $c=1.2 \mathrm{~mm}$ (p. 67), and $h=30 \mu \mathrm{m}$ (p. 82). Substituting these values in equations 3.3 to 3.6 and then those results into 3.2 gives a calculated nominal resonant frequency for the actual cavity of approximately $77.670 \mathrm{GHz}$, however, given the possible $10 \%$ etching variation reported in Section 4.1 (p. 63) the resonant frequency of an actual prototype cavity will fall between $70 \mathrm{GHz}$ and $83 \mathrm{GHz}$.

\subsection{Unloaded Quality Factor}

The unloaded quality factor for a perfectly hemispherical cavity resonator is well known and was given in equation 2.9. In theory, isotropically etching a cavity results in a perfect 
hemisphere with quality factors predicted by equation 2.9. As with the resonant frequency in the previous section, in practice this is not the case since the actual, isotropically etched cavities are not perfectly hemispherical (see Chapter 4). An actual micromachined cavity is an oblate hemispheroid with a solder layer and will have a different quality factor than given in equation 2.9 .

The unloaded quality factor for the actual cavity geometry can, however, be related to the unloaded quality factor for the perfect geometry. Since $\frac{\text { Cavity Volume }}{\text { Cavity Surface Area }} \propto$ $\frac{\text { Energy Stored }}{\text { Energy Lost }} \propto Q_{o}$ for both the actual and perfect geometries, dividing the two proportionality relations removes the proportionality constant. The resulting expression is

$$
\frac{Q_{0}^{\text {perfect cavity }}}{Q_{o}^{\text {actual cavity }}} \approx \frac{\left(\frac{\text { Perfect cavity volume }}{\text { Perfect cavity surface area }}\right)}{\left(\frac{\text { Actual cavity volume }}{\text { Actual cavity surface area }}\right)}
$$

Equation 3.7 approximates the actual unloaded quality factor since the volumes and surface areas are easily calculated and the unloaded quality factor for the perfectly hemispherical cavity was given previously in equation 2.9 .

The volume and surface area, $V_{o}$ and $S_{o}$, of the perfect hemisphere with radius $a$ are

$$
V_{o}=\frac{2}{3} \pi a^{3} \quad \text { and } \quad S_{o}=3 \pi a^{2}
$$

The volume and surface area, $V_{a}$ and $S_{a}$, of the actual oblate hemispherical cavity, with a solder layer, are

$$
V_{a}=\frac{2}{3} \pi a^{2} c+\pi a^{2} h \quad \text { and } \quad S_{a}=2 \pi a^{2}+2 \pi a h+\frac{\pi c^{2}}{2 e} \ln \left(\frac{1+e}{1-e}\right)
$$

where $a$ is the lateral radius, $c$ is the depth, $h$ is the solder thickness and the eccentricity, $e$, is $e=\sqrt{1-\frac{c^{2}}{a^{2}}}$. 
Rearranging equation 3.7, substituting equations 3.8 and 3.9 and then simplifying somewhat gives a reasonable approximation of the unloaded quality factor for the actual fully-assembled micromachined cavity:

$$
\begin{aligned}
Q_{o}^{\text {actual }} & \approx\left(\frac{V_{a}}{S_{a}}\right)\left(\frac{S_{o}}{V_{o}}\right) Q_{o}^{\text {perfect }} \\
& \approx\left(\frac{6 c+9 h}{4 a+4 h+\frac{c^{2}}{a e} \ln \left(\frac{1+e}{1-e}\right)}\right)\left(0.573 \frac{\eta}{\mathcal{R}}\right)
\end{aligned}
$$

where $\eta=377 \Omega$ is the intrinsic impedance of free space and $\mathcal{R}$ is the surface resistance of the cavity walls defined in equation 2.10 .

Nominally $a=1.7 \mathrm{~mm}$ (p. 59), $c=1.2 \mathrm{~mm}$ (p. 67), and $h=30 \mu \mathrm{m}$ (p. 82). Substituting these values in equation 3.10 gives a calculated nominal unloaded quality factor for the actual cavity of approximately 2064, although this assumes ideal surface resistance which will not occur in reality. Equation 2.10 for ideal surface resistance assumes dc conductivity, perfect shape, perfect solder bond, perfect alignment and no roughness. To determine the actual surface resistance, and hence a more realistic unloaded quality factor, these factors must be accurately quantified. This is, however, difficult if not impossible to do individually. Instead it is assumed that the roughness is the dominating factor and that the others may be ignored in comparison. There is a clear consensus in the literature that surface roughness can be a significant source of loss in cavity resonators [116]-[120], whereas frequency-dependent conductivity losses reported by some authors [121]-[123] has been disputed recently [124, 125]. Furthermore, our experiments demonstrate that alignment errors are less than $1 \%$ of the cavity size and therefore not a large perturbation to the overall geometry. Surface roughness, by comparison, can possibly double the surface resistance [126].

Surface roughness may be due to the roughness of the substrate or to the roughness 


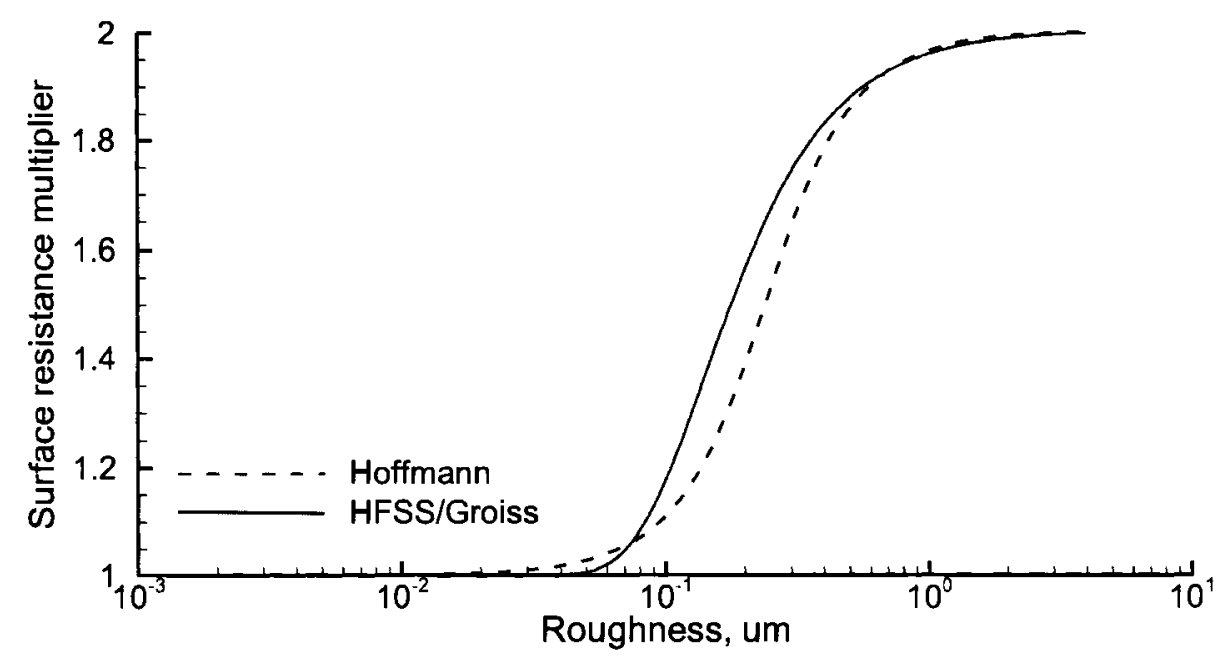

Figure 3.2: Theoretical surface resistance multiplier, $K$, due to roughness published by both Hoffmann [127] and Groiss/HFSS [126, 128].

of the conductor deposition but regardless will increase the losses in the cavity. This is especially true as the frequency increases since the current penetrates less into the metal (smaller skin depth) and more current is forced to follow the roughness profile resulting in more power loss (higher surface resistance) than if there was no roughness [129]. At $77 \mathrm{GHz}$ the skin depth is $0.283 \mu \mathrm{m}$ and hence a small amount of roughness may be significant. Real surface roughness profiles are complex three-dimensional random surfaces that are extremely difficult to model thus roughness is usually characterized as a root-meansquare (rms) roughness deviation measurement from the mean surface [130], however, this model assumes periodic roughness across the surface which is overly simplistic. Despite the inaccuracy of rms surface roughness, the impact of roughness on quality factor can be assessed without knowing the exact roughness profile. The actual surface resistance can be expressed as a multiple of the ideal surface resistance or $\mathcal{R}_{\text {actual }}=K \times \mathcal{R}_{\text {ideal }}$ where $K$ has been reported as $1+\frac{2}{\pi} \arctan \left(\frac{1.4 \psi^{2}}{\delta_{s}^{2}}\right)[127]$ or $1+e^{-\left(\frac{\delta_{s}}{2 \psi}\right)^{1.6}}[126,128]$ where $\psi$ is the surface roughness and $\delta_{s}$ is the skin depth. These are plotted in Figure 3.2 and are vir- 
tually the same (plotted at $77 \mathrm{GHz}$ with a skin depth of $0.283 \mu \mathrm{m}$ ). For smooth surfaces the actual surface resistance is ideal and then it increases as the roughness approaches and then exceeds a skin depth. Then the actual surface resistance reaches double the ideal surface resistance after which a further increase in roughness does not increase the loss. Therefore the worst case surface resistance is double the ideal value and this can be used to calculate the worst case quality factor with realistic losses accounted for. This worst case value is $Q_{o}=1036$.

The actual surface resistance is impossible to know, but since it can be up to twice the ideal value, 1.5 times ideal is taken as a more realistic value for the calculations and simulations. Accordingly, substituting $1.5 \mathcal{R}$ into equation 3.10 with the nominal parameters of $a=1.7 \mathrm{~mm}, c=1.2 \mathrm{~mm}$, and $h=30 \mu \mathrm{m}$ gives a calculated unloaded quality factor of approximately $Q_{o}=1381$ for a cavity with realistic losses.

\subsection{Unloaded Simulations}

The calculated resonant frequency and unloaded quality factor (from the previous two sections) were verified by unloaded finite element simulations. These unloaded simulations are known as "eigenmode simulations" because the cavity is simulated unconnected to the outside world with no sources included in the geometry. With no sources in the simulation, the resonances or "eigenmodes" of the geometry are solved for, including their associated unloaded quality factors and electromagnetic fields.

Both a nominal perfectly hemispherical cavity and a nominal micromachined cavity (an oblate hemispheroid soldered to alumina), shown in Figure 3.3, were simulated using eigenmode analysis in Ansoft HFSS . The simulations used a full three dimensional finite element method (FEM) with automatic adaptive mesh generation and refinement. Both cavities were filled with a air $\left(\epsilon_{r}=1.006, \mu_{r}=1.0000004\right)$. All the walls of each cavity 


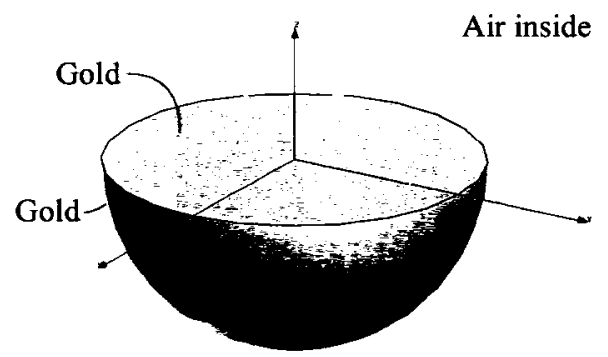

Perfect Hemispherical Cavity

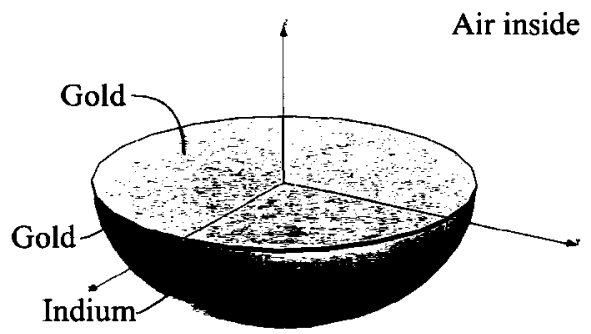

Actual Micromachined Cavity

Figure 3.3: Unloaded eigenmode simulation geometries.

\begin{tabular}{|c|c||cc|cc|}
\hline \multirow{2}{*}{ Cavity Geometry } & \multirow{2}{*}{ Mode } & \multicolumn{2}{c|}{ Nominal Frequency, GHz } & \multicolumn{2}{c|}{ Nominal Unloaded $Q$} \\
\cline { 3 - 6 } & & Calculated & Simulated & Calculated & Simulated \\
\hline \hline & $T M_{011}$ & 77.002 & 76.904 & 1671 & 1668 \\
Perfect Hemisphere & $T M_{m 21}$ & 108.600 & 108.400 & - & 1920 \\
(radius 1.7 mm) & $T E_{m 11}$ & 126.083 & 126.030 & - & 2264 \\
& $T M_{m 31}$ & 139.553 & 139.190 & - & 2049 \\
\hline \hline Micromachined: & $T M_{011}$ & 77.670 & 77.682 & 1381 & 1392 \\
Oblate Hemi./Solder & $T M_{m 21}$ & - & 114.870 & - & 1595 \\
(radius 1.7 mm & $T E_{m 11}$ & - & 149.080 & - & 1725 \\
depth 1.2 mm) & $T M_{m 31}$ & - & 152.730 & - & 1994 \\
\hline
\end{tabular}

Table 3.1: Simulated unloaded resonant frequencies and unloaded quality factors (with realistic surface resistance of 1.5 times ideal).

were gold $\left(\sigma=4.098 \times 10^{7} \mathrm{~S} / \mathrm{m}\right)$, however, the actual micromachined cavity also included a cylindrical face of indium solder with a conductivity of $1.195 \times 10^{7} \mathrm{~S} / \mathrm{m}$.

The resulting simulated unloaded resonant modes and unloaded quality factors are given in Table 3.1 with the nominal calculated values from Sections 2.2, 3.1 and 3.2 also included for comparison. The eigenmode simulations also provide simulated field patterns for the unloaded modes. The electric and magnetic fields for the fundamental $T M_{011}$ mode are shown in Figure 3.4 for the perfectly hemispherical cavity and in Figure 3.5 for the actual micromachined cavity. Note that the previously calculated fields of Figure 2.12 are the same as the simulated fields of Figure 3.4 and even similar to those of Figure 3.5. 


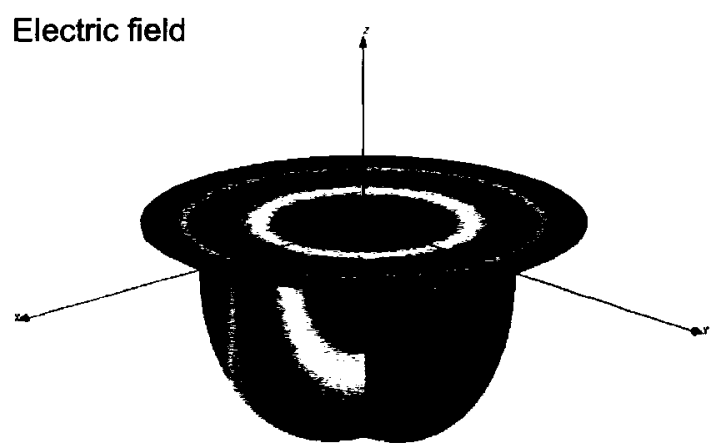

High 1 Low

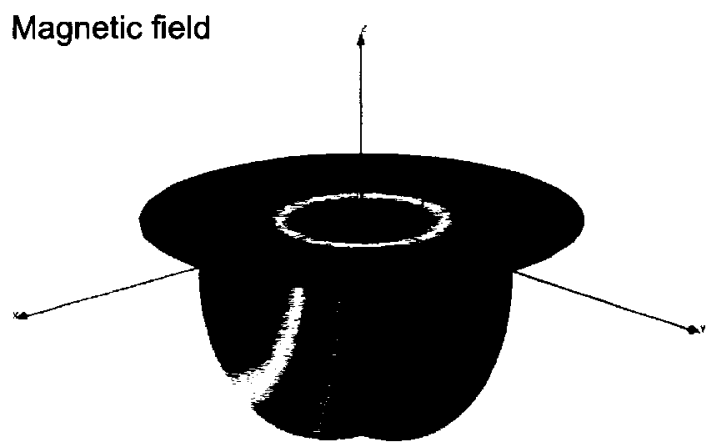

High $\square$ Low

Figure 3.4: Unloaded eigenmode simulation of the $T M_{011}$ electric and magnetic fields in a perfect hemispherical cavity.

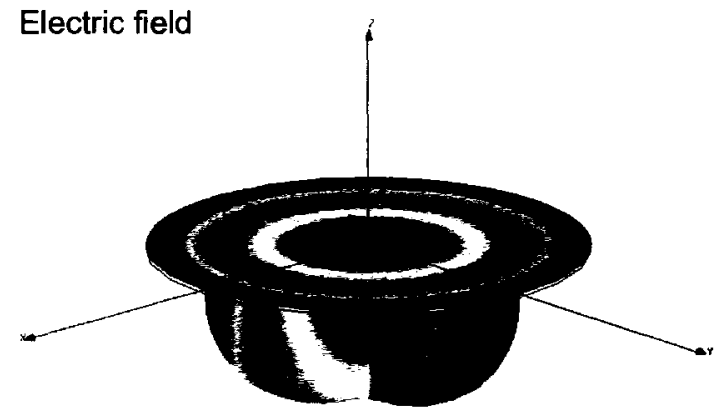

High 1 Low

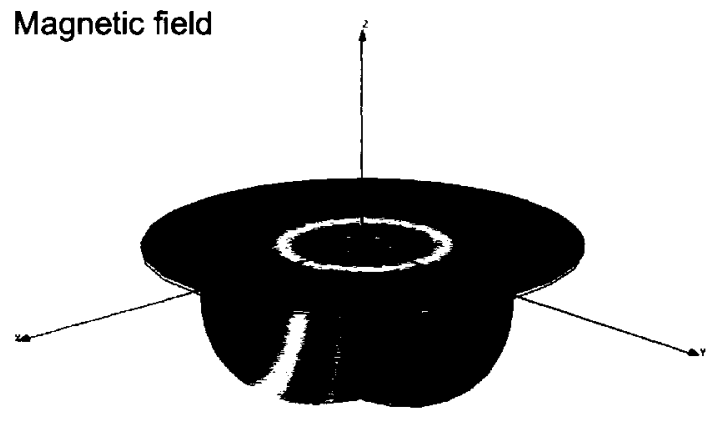

High 1 Low

Figure 3.5: Unloaded eigenmode simulation of the $T M_{011}$ electric and magnetic fields in an oblate hemispheroid micromachined cavity with a solder layer. 


\subsection{Loaded Quality Factor}

Once a means is introduced to couple the electromagnetic fields from the resonator to a transmission line, the resonator boundary conditions are no longer the same as the unloaded analysis of Sections 3.1 and 3.2. The new quality factor of a coupled resonator is called the loaded quality factor, $Q_{l}$, and will be lower than $Q_{o}$ due to the additional losses from the coupling means and also the external loading. The specifics of the coupling determines how much lower $Q_{l}$ is than $Q_{o}$. The weaker the coupling the higher the $Q_{l}$, which in turn lowers the phase noise when used to stabilize an oscillator.

Aperture coupling was introduced in Section 2.2 and there are literally hundreds of publications on it [131]. One in particular, namely "equivalent energy aperture coupling theory," has been used to calculate the loaded quality factor directly for a cylindrical waveguide cavity coupled to non-dispersive microstrip [132, 133]. Equivalent energy aperture coupling has also been used more recently to analyze an LTCC cavity [13]. For this research, equivalent energy aperture coupling theory was modified to calculate the loaded quality factor directly for a micromachined hemispherical cavity that is aperture coupled to dispersive microstrip. Furthermore, the loaded quality factor is expressed as a function of aperture radius which can be used to control the amount of coupling between the cavity and an active device in an oscillator.

Equivalent energy aperture coupling theory evaluates coupling between a cavity and a transmission line by considering two symmetrical aperture coupling problems:

1. Coupling between two identical transmission lines due to a small aperture in a common wall, characterized by a normalized reactance $x$.

2. Coupling between two identical cavities by the same aperture, characterized by a coupling factor $k$. 


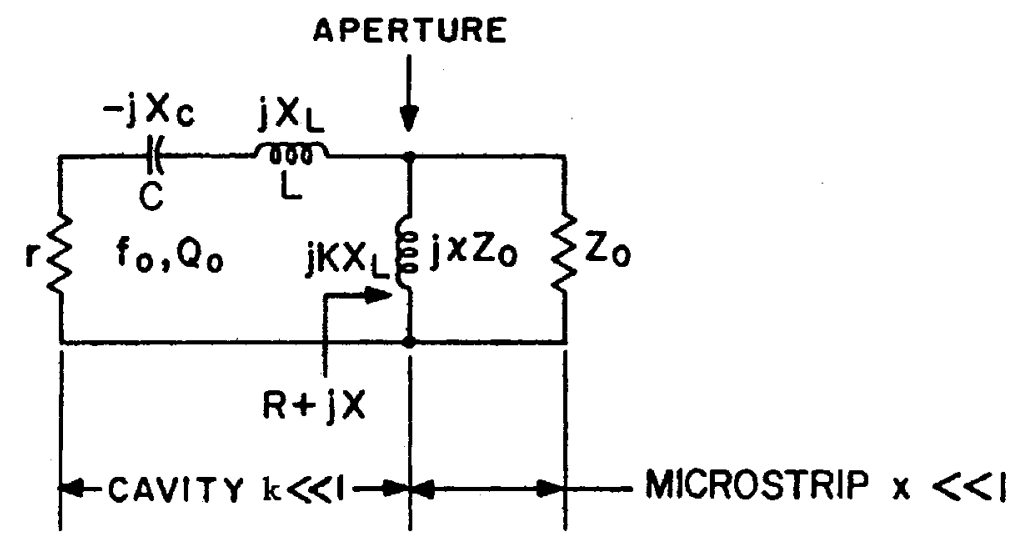

Figure 3.6: Equivalent circuit model for a cavity aperture coupled to microstrip. (diagram from [132]).

Then the loaded quality factor is $[134,135]$

$$
\frac{1}{Q_{l}}=\frac{1}{Q_{o}}+k x
$$

Equation 3.11 is the classic expression $\frac{1}{Q_{l}}=\frac{1}{Q_{o}}+\frac{1}{Q_{e}}$ with $\frac{1}{Q_{e}}=k x$, which can be explained using the equivalent circuit model shown in Figure 3.6. The aperture reactance can be modeled either as $k X_{L}$ (in terms of the coupling factor between two equivalent aperture coupled cavities) or as $x Z_{o}$ (in terms of the normalized reactance between two aperture coupled microstrips). The aperture is the same in both scenarios so $k X_{L}=x Z_{o}$ and multiplying both sides of this equality by $x$ gives $k x X_{L}=x^{2} Z_{o}$. Since the impedance transformed into the cavity by the aperture is $R+j X=Z_{o} x^{2}+j Z_{o} x$ then $k x X_{L}=R$ and hence $k x=\frac{R}{X_{L}}=\frac{1}{Q_{e}}$.

The cavity to cavity coupling coefficient, $k$, is evaluated in terms of a "cavity effective volume", $V_{c a v}$, and an "aperture effective volume", $V_{a p}$. The cavity effective volume, $V_{c a v}$, is defined as the volume that, when uniformly filled with the field existing at the aperture, stores an amount of energy equivalent to that stored by the actual cavity. The aperture effective volume, $V_{a p}$, is related to the polarizability of the aperture (see Section 2.2). 
The coupling coefficient for the two coupled cavities, in terms of effective volumes, is then $[134]$

$$
k=\frac{1}{4} \frac{V_{a p}}{V_{c a v}}
$$

This coupling coefficient is calculated for two aperture-coupled perfectly hemispherical cavities rather than for two aperture-coupled oblate hemispherical cavities (with a solder layer). It will be shown on p. 46 that the coupling coefficients are almost the same and thus using the field components of a perfect hemisphere, not to mention spherical instead of oblate spheroidal coordinates for the integrations, greatly simplifies the calculations. The approximation is valid because the total magnetic field in a perfectly hemispherical cavity is approximately the same as an oblate hemispherical cavity since the majority of the field exists in the upper portion near the $x y$ plane for both geometries. Indeed, at the $x y$ plane the field components are the same since the cross-section is circular in both cases thus the perfect hemisphere field components also can be used to find the field at the location of the aperture.

A circular aperture was chosen although other shapes could also excite the cavity, and perhaps even be more efficient at coupling for a given size, but coupling efficiency is not a design goal. Rather, the design goal is to maximize the loaded quality factor. Any aperture shape that gives an external quality factor at least two orders of magnitude higher than the unloaded quality factor is sufficient to maximize the loaded quality factor and another aperture shape that gives a higher external quality factor will not significantly improve the loaded quality factor. Furthermore, the expressions for polarizability and effective volume for a circular aperture are known, are closed-form, are simple and are independent of magnetic field orientation, whereas for non-circular shapes expressions are based on measurements or numerical techniques [136]-[141]. Finally, the orientation of non-circular apertures will affect the coupling $[142,143]$ whereas for circular apertures 
orientation is irrelevant due to symmetry. For all of these reasons a circular aperture shape is therefore chosen to couple the microstrip to the micromachined hemispherical cavity.

A three-quarter wavelength open-circuit microstrip stub is used to force a short circuit at the aperture so that the coupling is mostly magnetic. The stub essentially removes the electric field at the centre aperture, and although a small electric field will exist between the centre of the aperture and its outer edge, this field is assumed negligible compared to the strength of the magnetic field. Furthermore, the aperture is located at a position of high magnetic field inside the cavity (and low electric field) to ensure mostly magnetic coupling from the microstrip to the component of the cavity $\vec{H}$ field tangential to the plane of the aperture.

Since the coupling is therefore only magnetic, only the magnetic polarizability need be considered, where, the magnetic polarizability is the quotient of an equivalent dipole moment over the incident field intensity [100]. In this definition, the polarizability includes a permittivity or permeability term. If the polarizability is re-defined as the quotient of the equivalent dipole moment over the incident flux density of the field, then polarizability has the units of volume [134]. Thus, the aperture polarizability can be then related to the "aperture effective volume". For circular apertures of radius $r_{a}$ there is no distinction between the two magnetic field orientations and the magnetic polarizability, $M$, and the corresponding effective volume for magnetic coupling, $V_{a p}$, are $[100,144,134]$

$$
\begin{aligned}
M & =\frac{4}{3} r_{a}^{3} \\
V_{a p} & =\frac{16}{3} r_{a}^{3}
\end{aligned}
$$

The cavity effective volume, $V_{c a v}$, is the effective volume that when uniformly filled with the field existing at the position of the aperture, stores an amount of energy that is 
the same amount of energy stored by the actual cavity. Mathematically this is [133]

$$
\frac{1}{2} \mu\left|\vec{H}_{a p}\right|^{2} V_{c a v}=\frac{1}{2} \mu \int_{c a v}\left|\vec{H}_{\phi}\right|^{2} d V
$$

where $\vec{H}_{a p}$ is the tangential magnetic field at the location of the aperture but without the aperture present. On each side of equation 3.15 the $\frac{1}{2} \mu$ can be canceled, and thus the right side becomes

$$
\begin{aligned}
\int_{c a v}\left|H_{\phi}\right|^{2} d V & =\int_{r=0}^{a} \int_{\theta=0}^{\pi} \int_{\phi=0}^{2 \pi}\left(\frac{\sin \theta}{u_{11}^{\prime} r^{2}}\left[a \sin \left(u_{11}^{\prime} \frac{r}{a}\right)-u_{11}^{\prime} r \cos \left(u_{11}^{\prime} \frac{r}{a}\right)\right]\right)^{2} r^{2} \sin \theta d \phi d \theta d r \\
& =\frac{2 \pi a}{3 u_{11}^{\prime 2}}\left(u_{11}^{\prime 2}+u_{11}^{\prime} \cos u_{11}^{\prime} \sin u_{11}^{\prime}+2 \cos ^{2} u_{11}^{\prime}-2\right)
\end{aligned}
$$

Equation 3.16, and the magnetic field $\vec{H}_{a p}$ to follow, are calculated using the field components in equations 2.3 to 2.8 for a perfect hemisphere rather than for an oblate hemispheroid (see p. 42). Equation 3.16 is then substituted into equation 3.15 but to find $V_{\text {cav }}$ the magnetic field $\vec{H}_{a p}$ must also be known, and that requires knowledge of the aperture location.

The aperture is located at the position of maximum magnetic field so that the smallest possible aperture may be used for a desired amount of coupling and thus the small aperture assumption from Section 2.2 is maintained. The aperture location is solely a function of $r$ since $\vec{H}_{\phi}$ is independent of $\phi$ and the aperture is located on the $x y$ plane thus $\theta=\frac{\pi}{2}$. The radial location for maximum magnetic field, $r_{\max }$, is determined by solving $\frac{d}{d r} \vec{H}_{\phi}\left(r_{m a x}, \frac{\pi}{2}\right)=0$ for $r_{m a x}$. Thus differentiating equation 2.8 with $\theta=\frac{\pi}{2}$ gives

$$
\frac{d}{d r} \vec{H}_{\phi}\left(r, \theta=\frac{\pi}{2}\right)=\left(\frac{u_{11}^{\prime 2} r^{2}-2 a^{2}}{u_{11}^{\prime} a r^{3}}\right) \sin \left(u_{11}^{\prime} \frac{r}{a}\right)+\frac{2}{r^{2}} \cos \left(u_{11}^{\prime} \frac{r}{a}\right)
$$

Setting equation 3.17 equal to zero and solving for $r$ results in the location of maximum 
magnetic field, and hence the location of the aperture, at $r_{\max }=1.29 \mathrm{~mm}$. The result agrees with the maximum magnetic field location on the $x y$ plane shown in the field plots of Figure 2.12. Furthermore, Figure 2.12 shows that $r_{\max }$ is an absolute maximum and not a local maximum.

The tangential magnetic field at the centre of this aperture location, but without the aperture present, is $\vec{H}_{\phi}\left(r=r_{\max }, \theta=\frac{\pi}{2}\right)$ from equation 2.8 and the field can be assumed uniform over the aperture with magnitude equal to that at the centre. That is

$$
\left|\vec{H}_{a p}\right|=\frac{a \sin \left(u_{11}^{\prime} \frac{r_{\max }}{a}\right)-u_{11}^{\prime} r_{\max } \cos \left(u_{11}^{\prime} \frac{r_{\max }}{a}\right)}{u_{11}^{\prime} r_{\max }^{2}}
$$

This assumption is valid if the aperture is "small" (see Section 2.2), which is verified by comparing the maximum magnetic field at the centre with the average magnetic field across the aperture. The average field across the aperture is

$$
H_{a p, a v g}=\frac{1}{\pi r_{a}^{2}} \int_{r_{\text {max }}-r_{a}}^{r_{\max }+r_{a}} \int_{-\arctan \left(\frac{1}{r} \sqrt{r_{a}^{2}-\left(r-r_{\max }\right)^{2}}\right)}^{\arctan \left(\frac{1}{r} \sqrt{r_{a}^{2}-\left(r-r_{\max }\right)^{2}}\right)} H_{\phi}\left(r, \theta=\frac{\pi}{2}\right) r d \phi d r
$$

where $r_{a}$ is the aperture radius. For aperture radii of $0.12 \mathrm{~mm}$ and $0.18 \mathrm{~mm}$ the average field across the aperture is $700.6 \mathrm{~A} / \mathrm{m}$ and $696.5 \mathrm{~A} / \mathrm{m}$, respectively, which differs only slightly from the maximum field in the centre calculated by equation 3.18 which is $703.9 \mathrm{~A} / \mathrm{m}$. Accordingly, the small aperture assumption of a uniform field across the aperture equal to the maximum at the centre is sufficiently accurate.

With the tangential magnetic field at the aperture location and the total magnetic field known, the cavity effective volume can be calculated by substituting equations 3.16 and 3.18 into equation 3.15 :

$$
V_{c a v}=\frac{2 \pi a r_{\max }^{4}\left(u_{11}^{\prime 2}+u_{11}^{\prime} \cos u_{11}^{\prime} \sin u_{11}^{\prime}+2 \cos ^{2} u_{11}^{\prime}-2\right)}{3\left[a \sin \left(u_{11}^{\prime} \frac{r_{\max }}{a}\right)-u_{11}^{\prime} r_{\max } \cos \left(u_{11}^{\prime} \frac{r_{\max }}{a}\right)\right]^{2}}
$$


The cavity to cavity coupling coefficient, $k$, is calculated by substituting equation 3.20 and equation 3.14 into equation 3.12. As stated previously (p. 42) this is approximate since equation 3.20 uses the field components of a perfect hemisphere rather than for an oblate hemisphere with a solder layer. The accuracy of this approximation is evaluated by comparing this calculated coupling coefficient with a simulated coupling coefficient for two aperture-coupled oblate hemispherical cavities (with solder layers) derived from an eigenmode simulation in HFSS (with the same set up as in Section 3.3). The simulation results in two closely spaced resonant frequencies that are related to the coupling coefficient using $k_{s i m}=\frac{f_{2}^{2}-f_{1}^{2}}{f_{2}^{2}-f_{1}^{2}}$ [145]. The calculated coupling coefficient using equation 3.12 is $k=0.00130$ and the simulated coupling coefficient is $k_{s i m}=0.00134$. The similarity of these two results confirms that using the field components of a perfect hemisphere to approximate those of an oblate hemisphere in equation 3.20 is acceptable.

The cavity to cavity coupling coefficient, $k$, is not the only parameter needed to calculate the loaded quality factor using equation 3.11. The normalized reactance of the aperture, $x$, is also needed. This reactance is that of the aperture when coupling energy through a common ground plane of two identical microstrip lines, as shown in Figure 3.7. These microstrip lines are those used to feed the micromachined cavity.

Analyzing two microstrip lines that are aperture coupled through a common ground plane requires a fair degree of effort since the inhomogeneous dielectric surrounding the microstrip results in fields with hybrid modes [146]. A simpler approach is to approximate the microstrip with parallel plate waveguide (PPWG) having an effective width, $W_{\text {eff }}$, and an effective dielectric constant, $\epsilon_{e f f}$, as shown in Figure 3.7. This will approximate the microstrip if $W_{\text {eff }}$ and $\epsilon_{\text {eff }}$ are chosen such that the PPWG has the same characteristic impedance and phase velocity as the fundamental quasi-TEM microstrip mode. Using the microstrip parameters chosen in Section $4.2\left(Z_{o}, w_{\mu s t r i p}, h_{\mu s t r i p}\right.$ and $\left.\epsilon_{r}\right)$, 


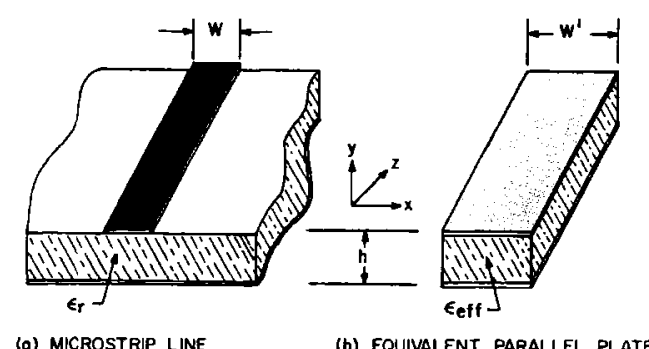

(b) EQUIVALENT PARALLEL PLATE

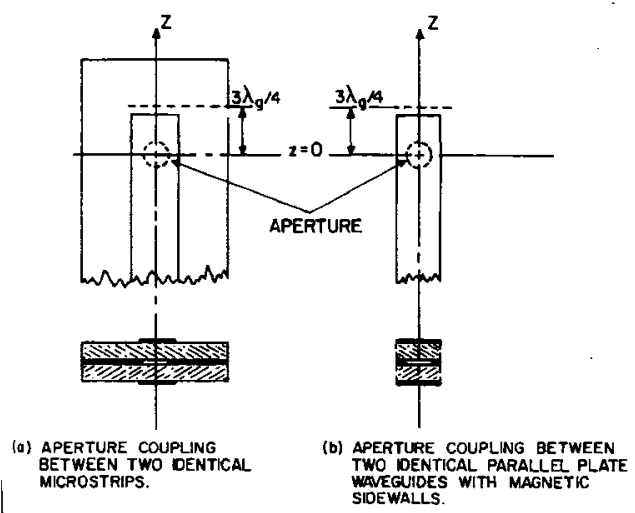

Figure 3.7: Aperture coupling between two microstrips and the equivalent parallel plate waveguide model (diagram from [133]).

\begin{tabular}{|r|c|c|c|c|c|c|}
\hline & {$[149]$} & {$[11]$} & {$[150]$} & {$[151]$} & {$[152]$} & Average \\
\hline Quasi-static, $\epsilon_{\text {eff,dc }}$ & 6.651 & 6.693 & 6.678 & & & 6.674 \\
Including dispersion, $\epsilon_{\text {eff }}$ & & & & 7.265 & 7.253 & 7.259 \\
\hline
\end{tabular}

Table 3.2: Microstrip effective dielectric constants calculated using published methods.

$W_{\text {eff }}$ is given by $[147,148]$

$$
W_{\text {eff }}=w_{\mu s t r i p}+\left(\frac{h_{\mu s t r i p}}{Z_{o}} \frac{377}{\sqrt{\epsilon_{e f f, d c}}}-w_{\mu s t r i p}\right)\left(1+\frac{2 w_{\mu s t r i p} f \sqrt{\epsilon_{r}}}{3 \times 10^{8}}\right)^{-1}
$$

There is no single expression in the literature to calculate $\epsilon_{\text {eff }}$ for quasi-static and dispersive conditions and thus the average of the multiple approaches shown in Table 3.2 are used in equations 3.21 to equation 3.25 .

The equivalent PPWG model for the microstrip can be used to approximate the aperture reactance by considering two aperture coupled parallel plate waveguides. This equivalent geometry is shown in Figure 3.7. The approximate normalized reactance is [144]

$$
x=\left|\frac{T}{2 j(1-T)}\right|
$$


where the transmission coefficient, $T$, is $[133,144]$

$$
T=\frac{2 \pi j}{S \lambda_{g}} \cdot M \cdot 2 H_{o}^{2}
$$

Equation 3.23 assumes no electric field at the aperture (due to the $\frac{3 \lambda}{4}$ open circuit stub) and a magnetic field component perpendicular to the $z$-axis with magnitude $2 H_{o}^{2}$ uniformly over the aperture (small aperture assumption). Also, $M$ is the polarizability of the aperture given by equation $3.13, \lambda_{g}=\frac{\lambda_{o}}{\sqrt{\epsilon_{e f f}}}$ is the guided wavelength and $S$ is a normalizing factor given by $[133,144]$

$$
S=\frac{1}{\eta} \int_{\text {guide }} \vec{n} \cdot\left(\vec{E}_{t} \times \vec{H}_{r}\right) d s=\frac{1}{\eta} \int_{0}^{W^{\prime}} \int_{0}^{h}\left|E_{t} \| H_{t}\right| d y d x=H_{o}^{2} h w_{e f f}
$$

Thus substituting equation 3.23 into 3.22 results in the normalized aperture reactance as a function of aperture radius $r_{a}$

$$
x=\left|\frac{4 \pi r_{a}^{3} \sqrt{\epsilon_{e f f}}}{3 h W_{e f f} \lambda_{o}-j 8 \pi r_{a}^{3} \sqrt{\epsilon_{e f f}}}\right|
$$

where $W_{\text {eff }}$ is given by equation 3.21 and $\epsilon_{\text {eff }}$ is found in Table 3.2.

Therefore, with $Q_{o}, V_{a p}, V_{c a v}, k$ and $x$ now known, the loaded quality factor of a micromachined oblate hemispherical cavity, aperture coupled to microstrip, is calculated using equation 3.11 according to the following steps:

1. Calculate $Q_{o}$ using equation 3.10 :

$$
Q_{o}=\left(\frac{6 c+9 h}{4 a+4 h+\frac{c^{2}}{a e} \ln \left(\frac{1+e}{1-e}\right)}\right)\left(0.573 \frac{\eta}{\mathcal{R}}\right)
$$


2. Calculate $V_{a p}$ using equation 3.14 :

$$
V_{a p}=\frac{16}{3} r^{3}
$$

3. Calculate $V_{\text {cav }}$ using equation 3.20 :

$$
V_{c a v}=\frac{2 \pi a r_{\max }^{4}\left(u_{11}^{\prime 2}+u_{11}^{\prime} \cos u_{11}^{\prime} \sin u_{11}^{\prime}+2 \cos ^{2} u_{11}^{\prime}-2\right)}{3\left[a \sin \left(u_{11}^{\prime} \frac{r_{\max }}{a}\right)-u_{11}^{\prime} r_{\max } \cos \left(u_{11}^{\prime} \frac{r_{\max }}{a}\right)\right]^{2}}
$$

4. Calculate $k$ using equation 3.12 :

$$
k=\frac{1}{4} \frac{V_{a p}}{V_{c a v}}
$$

5. Calculate $x$ using equation 3.25 :

$$
x=\left|\frac{4 \pi r_{a}^{3} \sqrt{\epsilon_{e f f}}}{3 h W_{e f f} \lambda_{o}-j 8 \pi r_{a}^{3} \sqrt{\epsilon_{e f f}}}\right|
$$

6. Calculate $Q_{l}$ using rearranged equation 3.11 and the results from steps 1 to 5 :

$$
Q_{l}=\frac{Q_{o}}{1+Q_{o} k x}
$$

Virtually all of the parameters used to calculate the loaded quality factor using equation 3.26 (and steps 1 to 5) are predetermined except for the aperture radius. This results in a simple design curve, shown in Figure 3.8, where the loaded quality factor can be adjusted by solely changing the radius of the aperture. All of the other parameters are fixed (see also Chapter 4):

- The cavity radius, $a$, determines the frequency and the depth, $c$, is a result of the 


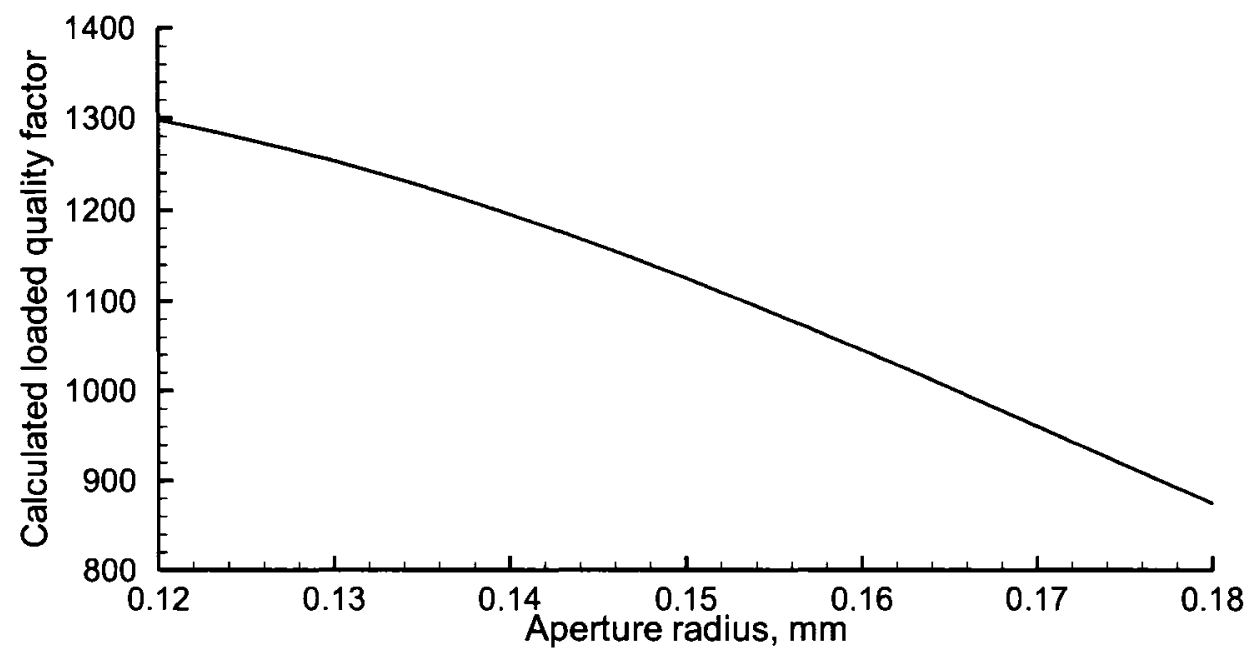

Figure 3.8: Calculated loaded quality factor as a function of circular aperture radius. self-limited micromachining,

- The solder thickness, $h$, is determined by the requirement for a good solder bond,

- The surface resistance, $\mathcal{R}$, is limited by choosing gold for the conductor metal, and

- The parameters for microstrip on alumina are chosen for a system characteristic impedance of $50 \Omega$ at the operating frequency and this also determines the parallel plate waveguide parameters $W_{\text {eff }}$ and $\epsilon_{e f f}$.

Therefore, the loaded quality factor $Q_{l}$ can be determined solely by the aperture radius $r_{a}$. This allows the loaded quality factor of the micromachined cavity to be easily and independently adjusted for a particular amount of coupling needed to sustain oscillations when the cavity is used with a specific active device in an oscillator. 


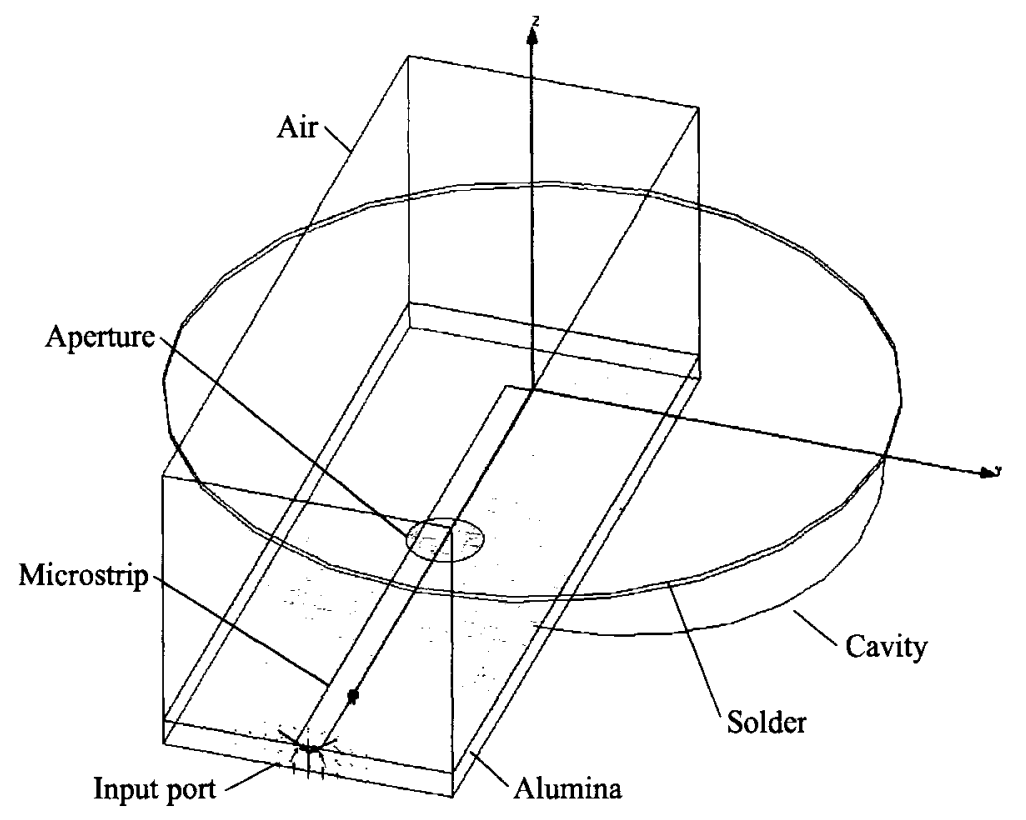

Figure 3.9: Micromachined cavity geometry for driven simulations.

\subsection{Loaded Simulations}

The calculated loaded quality factor (from the previous section) was verified by loaded finite element simulations. These loaded simulations are known as "driven simulations" because the cavity is simulated connected to the outside world using at least one source that drives the geometry. With sources in the simulation the S-parameters as a function of frequency are solved for, along with the associated electromagnetic fields.

The loaded quality factor of the aperture-coupled, micromachined oblate hemispherical cavity was simulated using driven analysis in Ansoft HFSS. The simulated geometry, shown in Figure 3.9, is driven by a $2 \mathrm{D}$ port solution at the input of the microstrip. The simulations use a full three dimensional finite element method (FEM) with automatic adaptive mesh generation and refinement, and they result in S-parameters and field plots. The resonant frequencies and quality factors can be computed from the S-parameters.

A literature survey reveals a variety of ways to compute quality factors from one-port 
resonator S-parameters such as the standard/overdetermined three points method [153, 154], the critical points method [155], the generalized loaded Q method [156] and the reactance change method [157]. The overdetermined three points method appears to be the most accurate [158] and is therefore chosen here. It is derived from the well-established "Q-circle" approach [159]-[161], but modified for modern network analyzers [153, 162], and embodied in the computer program QZERO for Windows [163].

The simulated micromachined cavity in Figure 3.9 has nominal parameters of radius $a=1.7 \mathrm{~mm}$ (p. 59), depth $c=1.2 \mathrm{~mm}$ (p. 67), gold walls $\left(\sigma=4.098 \times 10^{7} \mathrm{~S} / \mathrm{m}\right)$ and is filled with air $\left(\epsilon_{r}=1.006, \mu_{r}=1.0000004\right)$. The microstrip uses gold conductors and an alumina substrate $\left(127 \mu \mathrm{m}\right.$ thick, $\epsilon_{r}=9.9, \mu_{r}=1, \tan \delta=0.0001$, see p. 69) with air above. The indium solder layer has a conductivity of $1.195 \times 10^{7} \mathrm{~S} / \mathrm{m}$ and is $h=30 \mu \mathrm{m}$ thick (p. 82). The circular aperture in the microstrip ground plane/cavity top wall is a perfect $H$ boundary forcing continuity of the fields across the boundary.

This geometry was simulated around $77 \mathrm{GHz}$ for aperture radii varying from $0.12 \mathrm{~mm}$ to $0.18 \mathrm{~mm}$. The simulated results are shown in Figures 3.10 to 3.14. More specifically:

- The simulated $\mathrm{S}_{11}$ given in Figures 3.10 and 3.11 show that all of the cavities are undercoupled since none of the resonances on the Smith Chart encircle the $50 \Omega$ point at the centre. Furthermore, these results confirm that the smaller the aperture, the weaker the coupling.

- The broadband simulation shown in Figure 3.12 confirms only one resonance.

- The simulated electromagnetic fields given in Figure 3.13 confirm that the resonance is the desired $\mathrm{TM}_{011}$ mode.

- The simulated loaded quality factor, given in Figure 3.14 as a function of aperture radius, agrees with the calculated loaded quality factor from Section 3.4. 


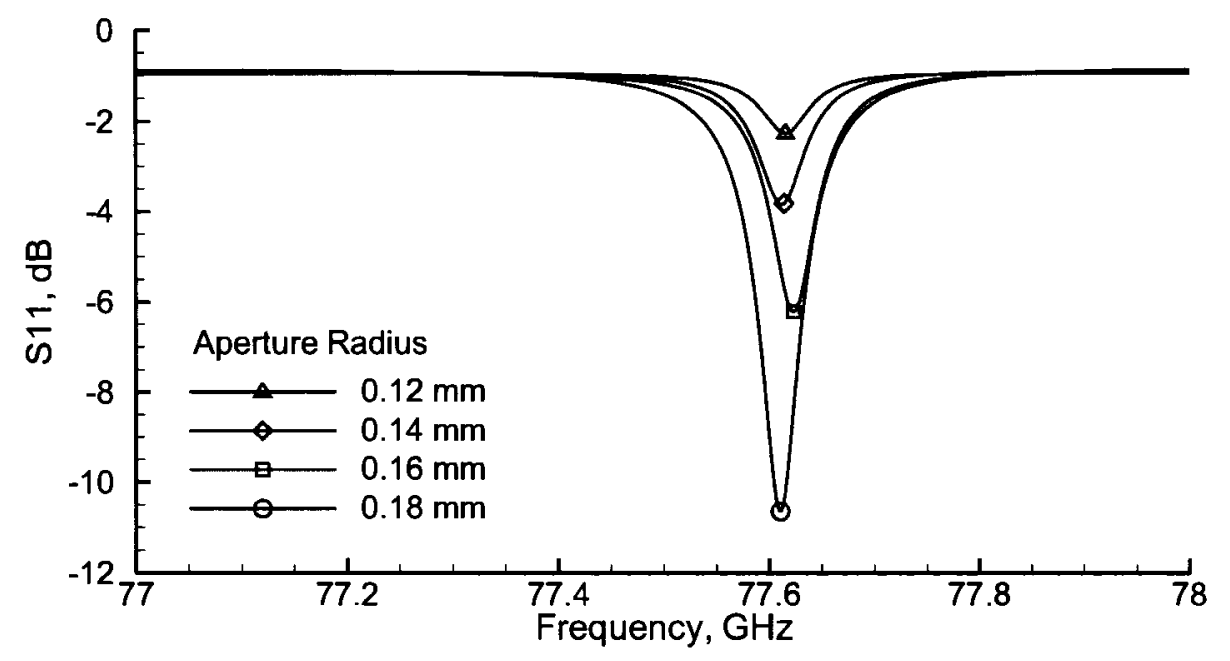

Figure 3.10: Simulated $S_{11}$ for micromachined cavities with aperture radii from $0.12 \mathrm{~mm}$ to $0.18 \mathrm{~mm}$.

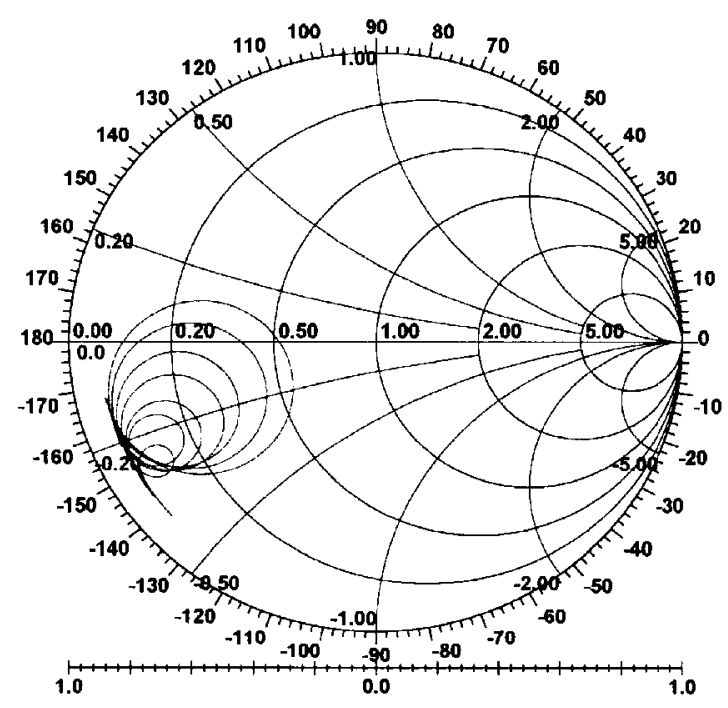

Figure 3.11: Simulated $S_{11}$ showing undercoupling for micromachined cavities with aperture radii from $0.12 \mathrm{~mm}$ (smallest circle) to $0.18 \mathrm{~mm}$ (largest circle) in steps of $0.01 \mathrm{~mm}$. 


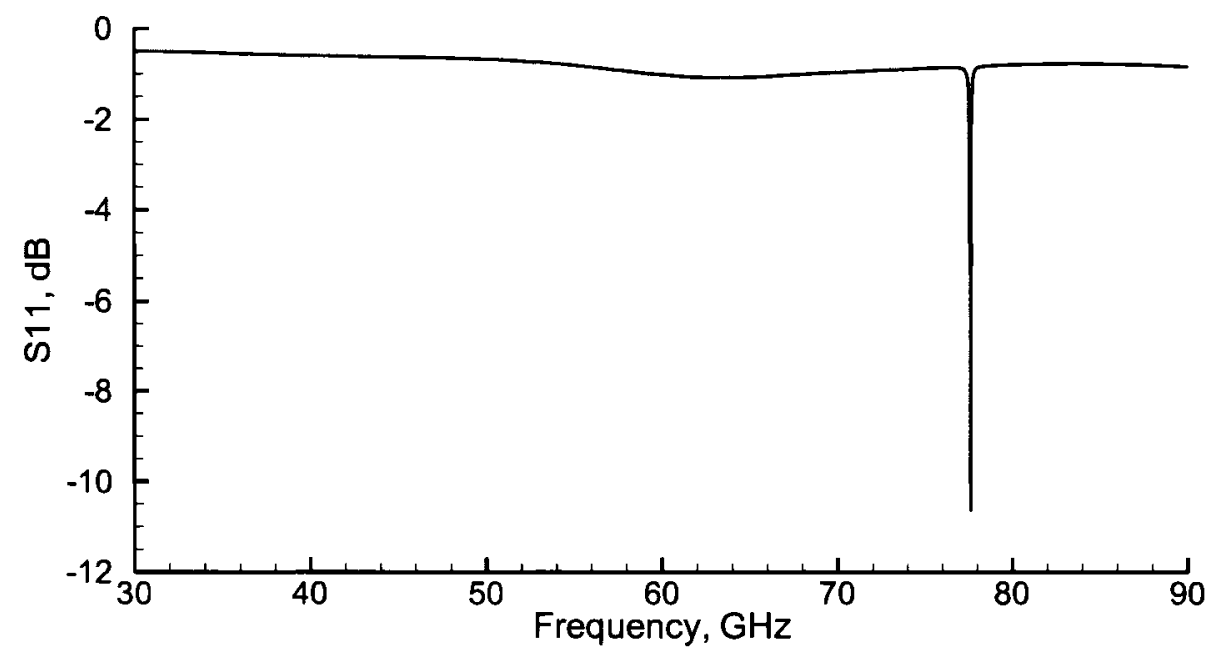

Figure 3.12: Broadband simulation of $\mathrm{S}_{11}$ for a micromachined cavity with an aperture radius of $0.18 \mathrm{~mm}$.

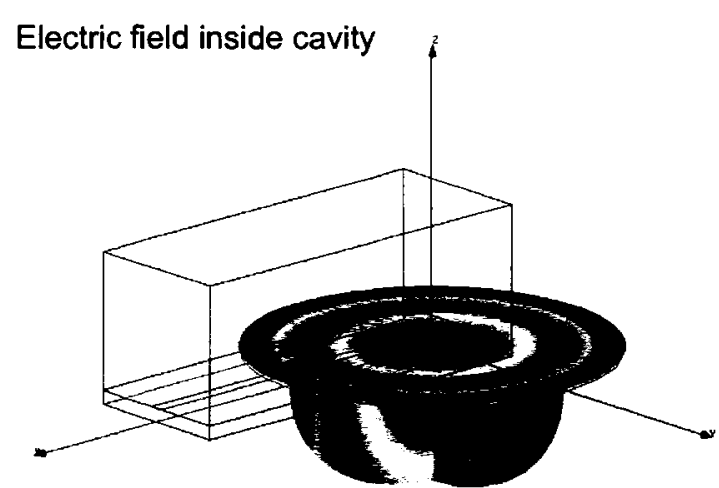

High 10 Low

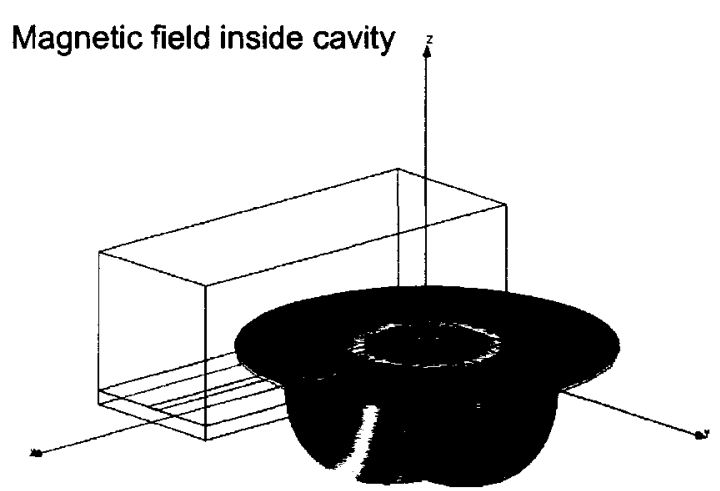

High IL Low

Figure 3.13: Simulated electromagnetic fields for a micromachined cavity with an aperture radius of $0.18 \mathrm{~mm}$. 


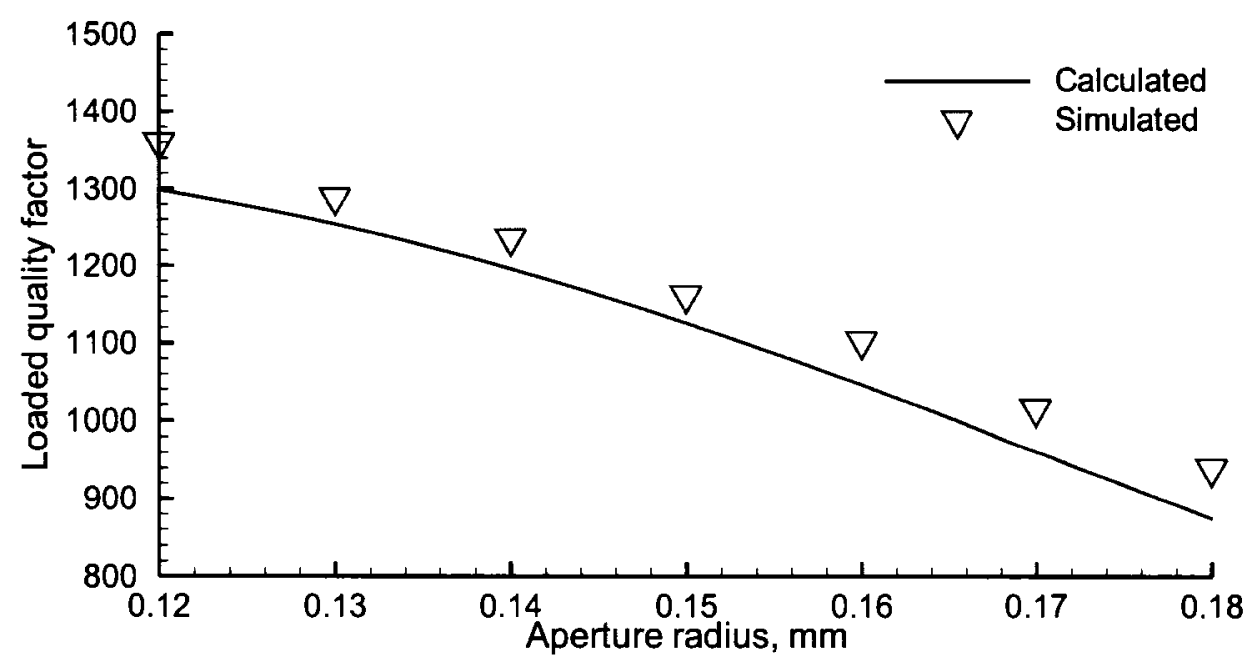

Figure 3.14: Simulated and calculated loaded quality v. aperture radius for a micromachined cavity.

In Figure 3.14 the loaded quality factor resulting from the simulations is slightly higher than that resulting from the calculations because the simulated coupling is likely weaker. For example, the short circuit imposed at the aperture by the $\frac{3 \lambda}{4}$ stub will not be perfect in the simulations because the simulated resonance is not exactly at $77 \mathrm{GHz}$ where the stub is designed to be $\frac{3 \lambda}{4}$. In the calculations this stub is assumed to be perfectly $\frac{3 \lambda}{4}$. Therefore, the calculations slightly overestimate the coupling and result in a slightly lower loaded quality factor compared to the simulations. 


\section{Chapter 4}

\section{Fabrication of Micromachined}

\section{Hemispherical Cavity Resonators}

This chapter describes the development of the steps to fabricate ${ }^{1}$ the micromachined hemispherical cavities designed in Chapter 3. Fabrication topics include micromachining experiments to etch the cavities, a microstrip feed substrate to excite the cavities, novel vialess probe pads for measurements and cavity assembly using indium solder. The resulting prototype micromachined hemispherical cavities are shown in Figure 4.29.

\subsection{Micromachining}

For a number of reasons, silicon wafers were chosen for the micromachined hemispherical cavity resonators. Firstly, isotropic etching of silicon (as in Section 2.3) is more established than etching a material more suited to millimeter wave applications, such as gallium arsenide $[111,164]$. Secondly, the silicon wafers are less costly thereby allowing more wafers for experimentation. Furthermore, the majority of micromachining today

\footnotetext{
${ }^{1}$ The fabrication of the cavities was managed by the author but performed by the Microfabrication Team at CRC.
} 


\begin{tabular}{|c|c|c|c|}
\hline Mask Material & Durability & Adhesion & Max. Radius \\
\hline \hline SiN, $2 \mu \mathrm{m}$ thick & Etched fast & No peeling off & $0.5 \mathrm{~mm}$ \\
Parylene & Not etched & Immediately peeled off & None \\
Parylene/adhesive & Not etched & Peeled off after $20-30 \mathrm{~min}$ & $0.5 \mathrm{~mm}$ \\
Polyethylene tape/adhesive & Not etched & Rarely peeled off & $>2.5 \mathrm{~mm}$ \\
$\mathrm{Cr} / \mathrm{Au}$ & Not etched & Peeled off after 20-30 min & $0.5 \mathrm{~mm}$ \\
$\mathrm{Cr} /$ Polyimide & Not etched & Immediately peeled off & None \\
\hline
\end{tabular}

Table 4.1: Various masks for long duration isotopic etching of silicon using HNA (HF : $\left.\mathrm{HNO}_{3}: \mathrm{CH}_{3} \mathrm{COOH}=2: 3: 3\right)$ at $50^{\circ} \mathrm{C}[112]$.

uses silicon and as such hemispherical cavity resonators may be easier to commercialize (eventually) if they are made using silicon. Therefore, commonly available $\langle 100\rangle$ wafers were selected and purchased from Virginia Semiconductor [104]. These wafers are 3 inches in diameter, have a custom thickness of $3 \mathrm{~mm}$ (allowing some flexibility when etching different cavity sizes) and are double side polished.

Of the isotropic etch formulations producing hemispherical structures that have been previously reported $[112,165,115,166]$, a two-step HNA etch [112] was chosen as a starting point to micromachine smooth and round hemispherical cavities. This two-step process first uses $2: 3: 3 \mathrm{HNA}$ at $50^{\circ} \mathrm{C}$ for the main etch, and then $1: 4: 3 \mathrm{HNA}$ at room temperature for a slight surface polish, if needed. It has been reported that the circularity of this two-step process varied less than $7.5 \%$ from wafer to wafer $[112,166]$.

In order to etch a specific geometry with this etchant formulation a portion of the wafer must be masked, however, isotropic etchants etch fast thus making masking somewhat difficult. If a shallow etch is required, then $\mathrm{SiO}_{2}$ is an effective mask, however, for deeper etching then $\mathrm{Si}_{3} \mathrm{~N}_{4}$ or $\mathrm{Au}$ is needed for an effective mask [103]. A key difficulty when etching large hemispherical structures is masking the strong isotropic silicon etchants for a long period of time. Several masking materials, summarized in Table 4.1, have been reported for long duration etching [112]. Most of the masks either etch too 
fast or peel off, thus limiting the size of the etched structures. For the main etch (HNA $2: 3: 3)$ polyethylene tape is the best in terms of adhesion and durability in the etchant. For the second polish etch (HNA $1: 4: 3$ ) SiN is sufficient.

A circular opening in the mask is required to etch a hemispherical cavity. Photolithography is used to define the circular opening in the polyethylene and SiN mask layers using aluminum to mask to polyethylene/SiN while they are being reactively ion etched. The size of the circular mask opening affects the sphericity of the etched cavity. Isotropic etching etches the same amount in all directions so if the circular mask opening is infinitely small then a perfect hemisphere results because the etching starts from the same point and etches the same amount. In contrast, when the circular mask opening has a finite size the isotropic etching does not start from the same point but still etches at the same rate in all directions starting from different points. Accordingly the depth of the resulting cavity will not be the same as the lateral radius at the surface of the wafer and the shape is an oblate hemispheroid. The larger the circular mask opening, the more the etched cavity deviates from a perfect hemisphere.

The etching time determines the overall size of the cavity and this is significant because the cavity size determines the frequency of operation (see Section 2.2). Traditionally, due to the fast etch rate, isotropic etching was difficult to control when attempting to etch precisely sized structures. Recently, however, it has been reported that isotropic etching can be self-limiting due to gas bubbles consisting of etch by-products that block the circular mask opening [167]. This blocking prevents transport of the etchant to the silicon surface thereby preventing further etching. As the spherical etch front grows during etching, so does the amount of the gas. Eventually, as shown in Figure 4.1, most of the spherical region under the mask window is filled with gas and the gas-generation rate exceeds the rate of gas leaving through the mask opening. Therefore, the gas prevents the etchant from diffusing to the silicon and the size of the etched cavity is thus 


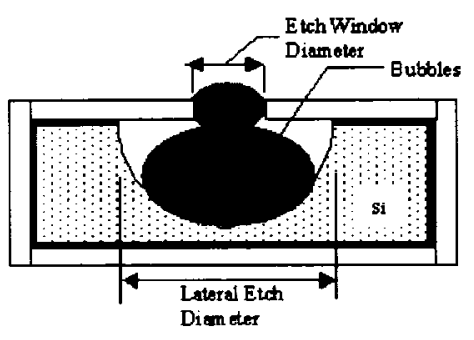

Figure 4.1: Self-limiting of the etch rate due to gas by-products [167].

mostly dependent on the size of the mask opening rather that the time in the etchant. Specifically, for a desired etch front diameter, the etching is self-limiting when the mask window diameter is [167]:

$$
\text { Mask window diameter }=\frac{\text { Etch front diameter }-400 \mu \mathrm{m}}{1.8}
$$

It has been reported that, for small cavities, the same lateral dimensions from cavity to cavity can be reproduced to within $4 \%$ using self-limited etching, assuming the etching conditions are the same. Therefore, the dimensions of the hemispherical cavity (and thus its frequency of operation) can be precisely controlled by a photolithographically defined circular opening in the mask, rather than attempting to "time" the etch, which is problematic at best.

Using self-limited isotropic etching, equation 4.1 gives an optimal mask opening diameter of $1.6671 \mathrm{~mm}$ to produce a cavity with $a=1.7004$, however, assuming a $1 \mu \mathrm{m}$ mask resolution, the actual mask opening diameter must be $1.667 \mathrm{~mm}$. Thus, using equation 4.1 , an actual mask opening diameter of $1.667 \mathrm{~mm}$ results in an actual cavity radius of $1.700 \mathrm{~mm}$, which using equation 2.2 , results in an actual resonant frequency of $77.002 \mathrm{GHz}$ instead of $77 \mathrm{GHz}$. This $2 \mathrm{MHz}$ change in resonant frequency is, however, negligible compared to the change due to variations in the cavity size from the isotropic etching (described in the sections that follow) and thus mask resolution may be neglected. 

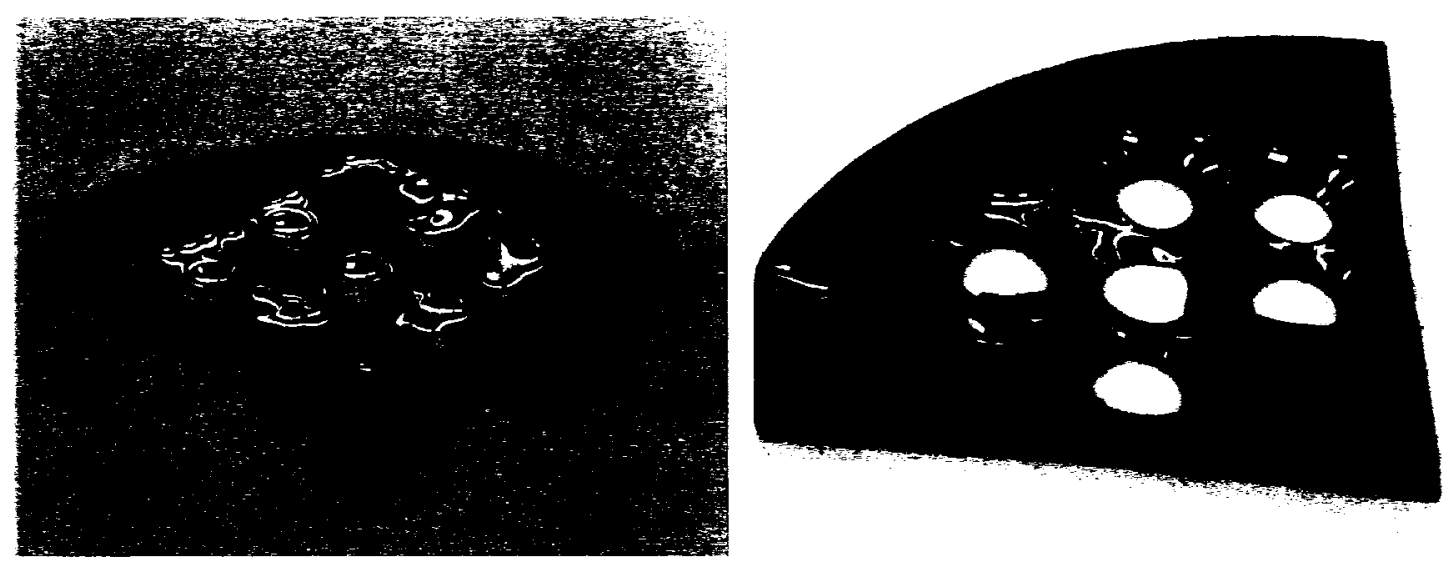

Figure 4.2: Initial results of isotropic etching experiments.

Therefore, the self-limited isotropic etching selected for the initial micromachining experiments was:

- Main 2:3:3 $\mathrm{HNA}$ etch at $50^{\circ} \mathrm{C}$ without agitation; masked using polyethylene tape

- Polish 1:4:3 HNA etch at room temperature without agitation; masked using SiN

where the HNA is $49 \%$ hydrofluoric, $70 \%$ nitric and $99 \%$ acetic acids.

Two masks were designed to test this base etching procedure (see appendix A for mask layouts). Together, the two masks defined 32 different etch mask opening sizes ranging from $1-3 \mathrm{~mm}$ in diameter corresponding to 32 possible etched cavities with diameters from $2.2-5.8 \mathrm{~mm}$. These cavities were then etched according to the base procedure above.

Initial results, shown in Figure 4.2 were not successful as the etching was uncontrolled. These failures were due to improperly applying the polyethylene tape causing etchant to enter through cracks. Also, sometimes the tape lifted slightly during etching allowing the etchant to pass along the surface of the wafer. Both of these factors caused uncontrolled etching.

These issues were corrected by etching the cavities soon after applying the tape thus 

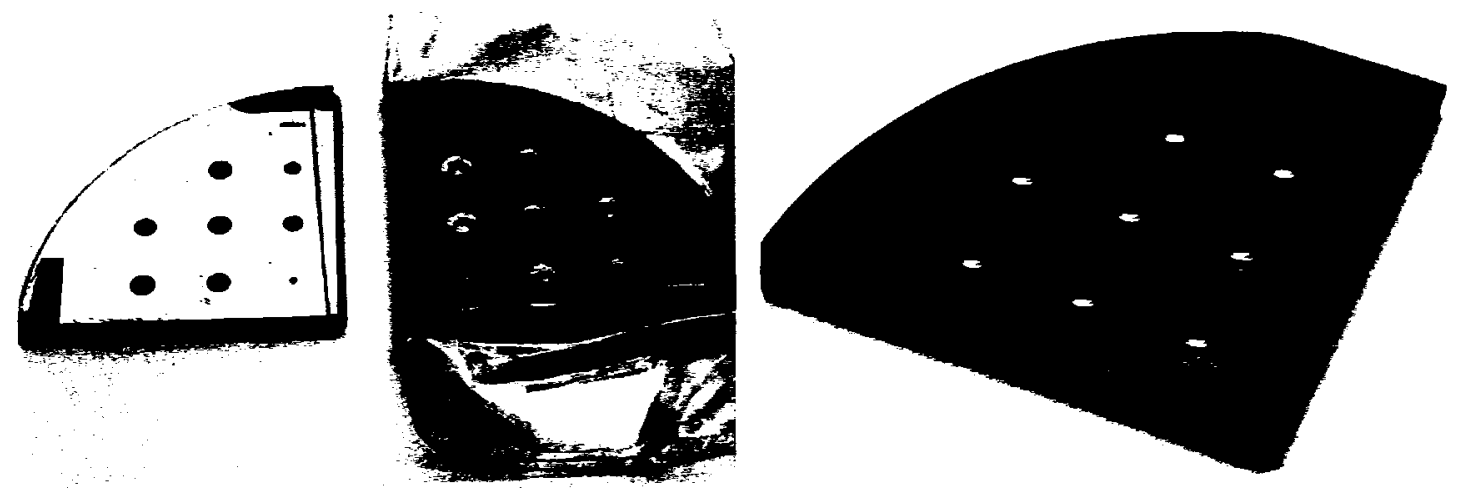

Figure 4.3: Patterned tape, aluminum mask and improved tape mask (left) resulting in the first repeatable cavities from isotropic etching experiments (right).

ensuring fresh adhesive and preventing lifting. Furthermore, extra tape was placed along the sides and over cracks. These improvements ensured that in the etchant was confined to the areas defined by the circular etch mask openings. Therefore, tape was first applied to the wafer and then the aluminum mask was used to pattern the circular etch mask openings. Then, the remaining sides and back of the wafer were covered with multiple layers of tape, as shown in Figure 4.3 (left). With the improved tape covering, repeatable etched cavities were produced as shown in Figure 4.3 (right).

The question of agitation was also addressed. Cavities were etched using the above base procedure both with and without agitation by a stir bar. Visual evidence suggests no difference in the shape or smoothness of the cavities. Examples are given in Figure 4.4.

Finally, the etch temperature was increased to $70^{\circ} \mathrm{C}$ and an etch bath with digital temperature feedback control was used to maintain a constant and accurate etchant temperature. The resulting cavities etched at $70^{\circ} \mathrm{C}$, shown in Figure 4.5, are much smoother than those etched at $50^{\circ} \mathrm{C}$.

The general size of the etched cavities can be see in Figure 4.6. Specific dimensions of the etched cavities were measured using a DekTak Profilometer as well as with a mi- 

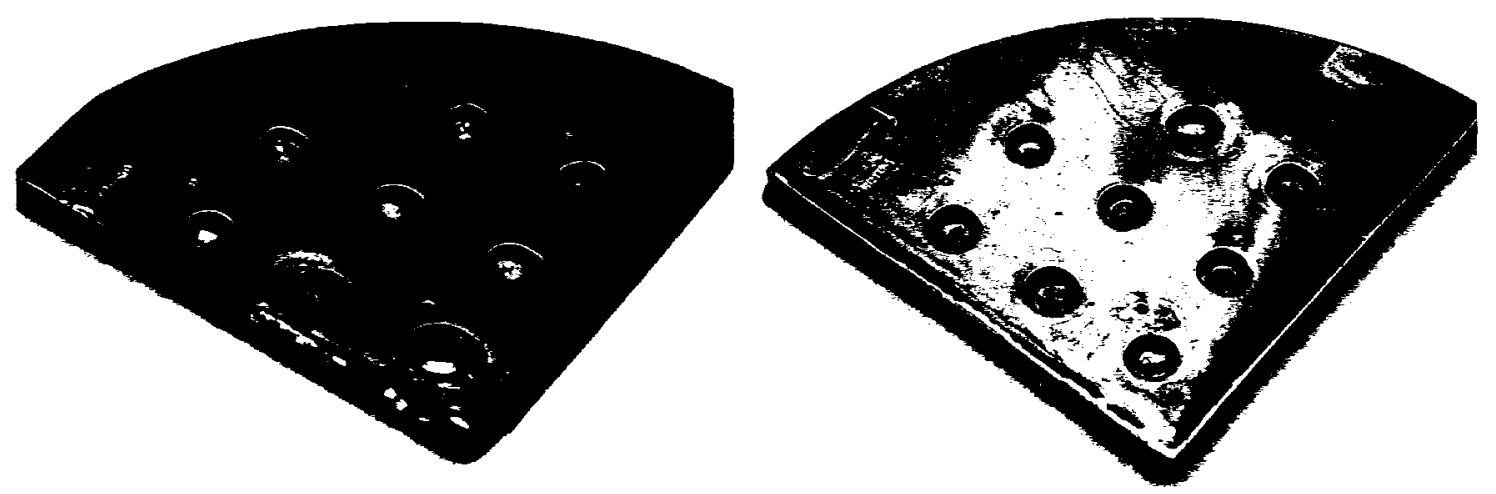

Figure 4.4: Etching experiments with (left) and without (right) agitation at $50^{\circ} \mathrm{C}$.
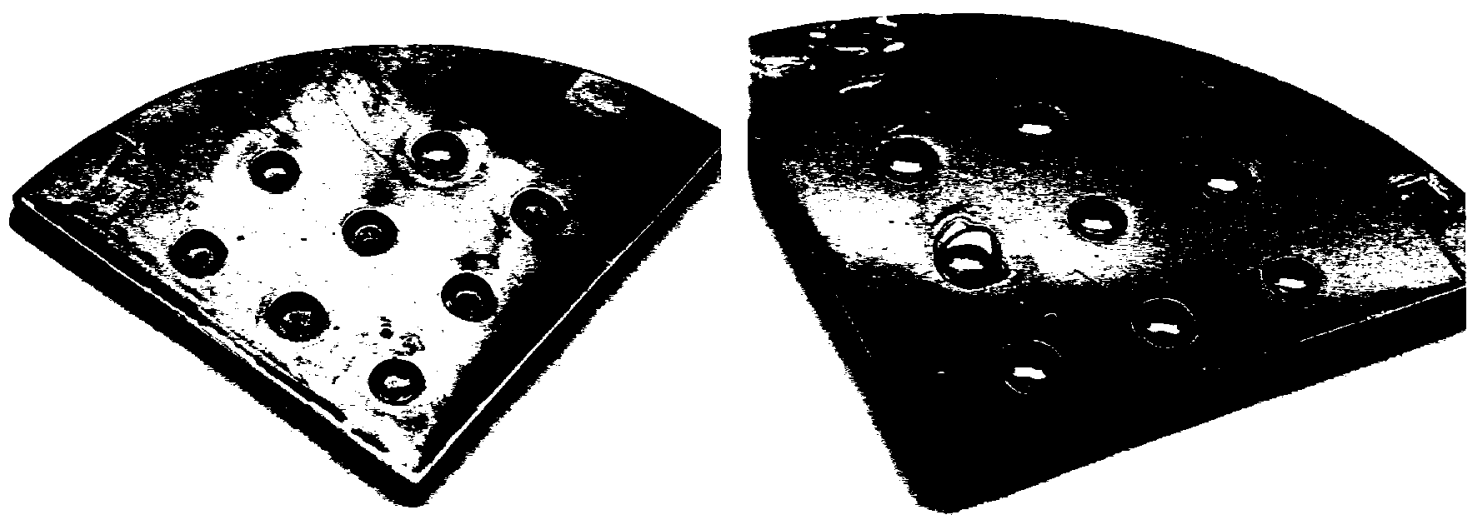

Figure 4.5: Etching experiments at $50^{\circ} \mathrm{C}$ (left) and $70^{\circ} \mathrm{C}$ (right).
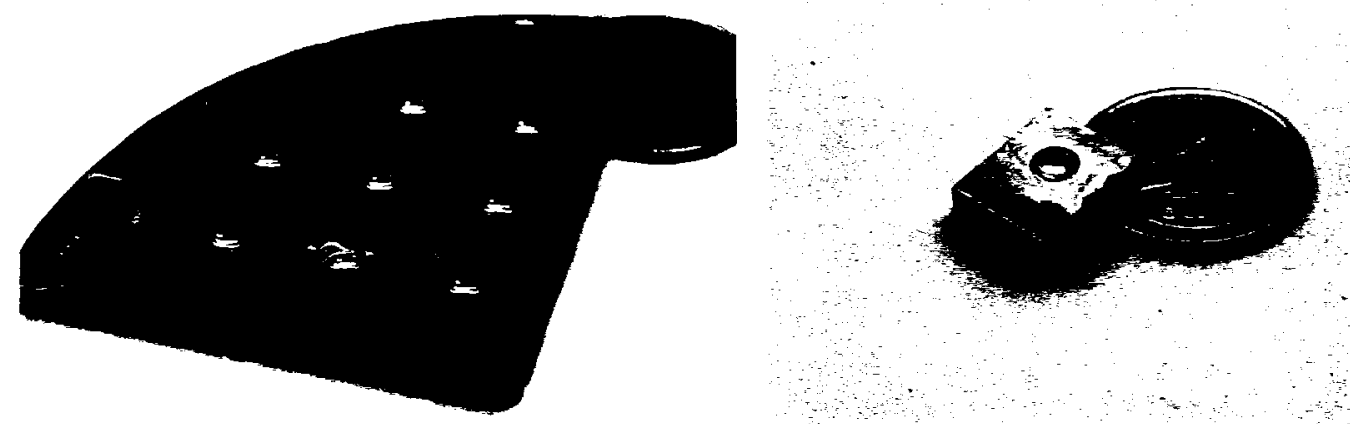

Figure 4.6: General size of the etched cavities. 
crometer attached to an optical microscope. Measurements of 13 representative cavities, with varying etch mask opening sizes, are shown in Figure 4.7. Based on these results, the isotropic etching is self-limiting with $75 \%$ of the samples having a measured radius between $1 \%-9 \%$ of their theoretical radius irrespective of etching time. These results are similar to those published by other researchers [167]. The cavities on the left of Figure $4.7(1,9-13)$ are early prototypes while those on the right $(2-8)$ are the best samples from the experiments $\left(70^{\circ} \mathrm{C}\right.$, no agitation).

In Figure 4.7, the measured depth is compared to the "average theoretical depth", which is an average of the maximum and minimum possible depths. The maximum possible depth is the maximum theoretical etched radius from self-limiting theory. This is the depth that would occur if all the etching started from a single point, which does not happen since the etching starts simultaneously everywhere in the mask opening. The minimum depth is the distance between the edge of the mask opening and the self-limited etch radius. This assumes that lateral etching starts at the edge of the mask opening immediately and continues until self-limiting occurs, however this is also not the case since the silicon exposed by the mask opening must be etched downward somewhat to expose edges for lateral etching. The true depth lies between this minimum and maximum, and is the minimum etched distance plus whatever initial etched depth is required in the mask opening to start isotropic lateral etching. According to the measurements in Figure 4.7 the average theoretical depth acceptably predicts the depth of the smaller cavities but less so for the larger cavities.

Therefore due to the mask opening, the depth and radius of the etched cavities are not the same and thus the cavities have an elliptical cross-section instead of circular. This is shown in Figure 4.8 where, for both a small and large etched cavity, the etched depth was measured at points across the cavities to generate a plot of the cross-sectional profile. These profiles were then compared to a plot of an equation of a perfect ellipse 

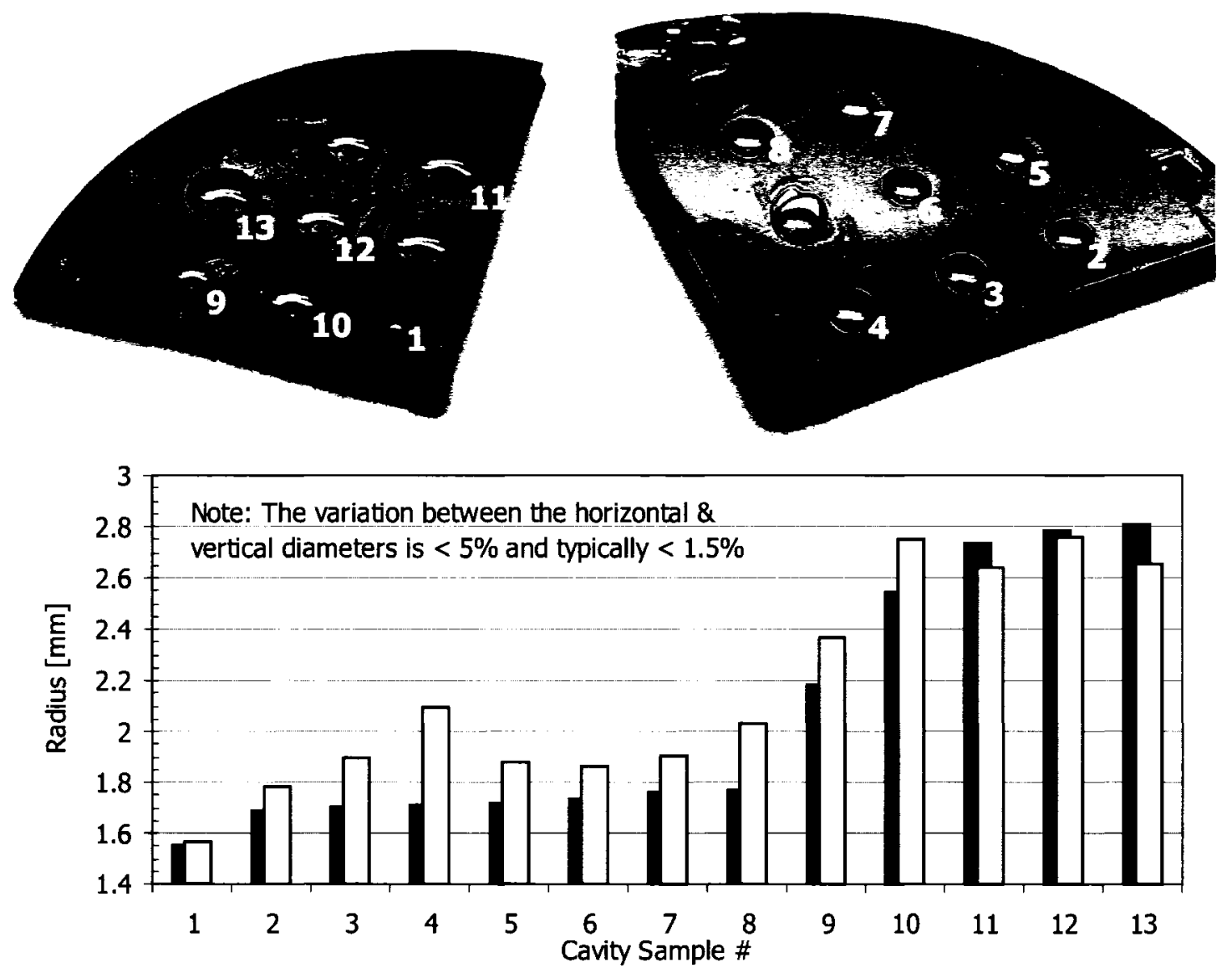

-Theoretical Minimum Radius [mm]

口Measured Horizontal Radius [mm]

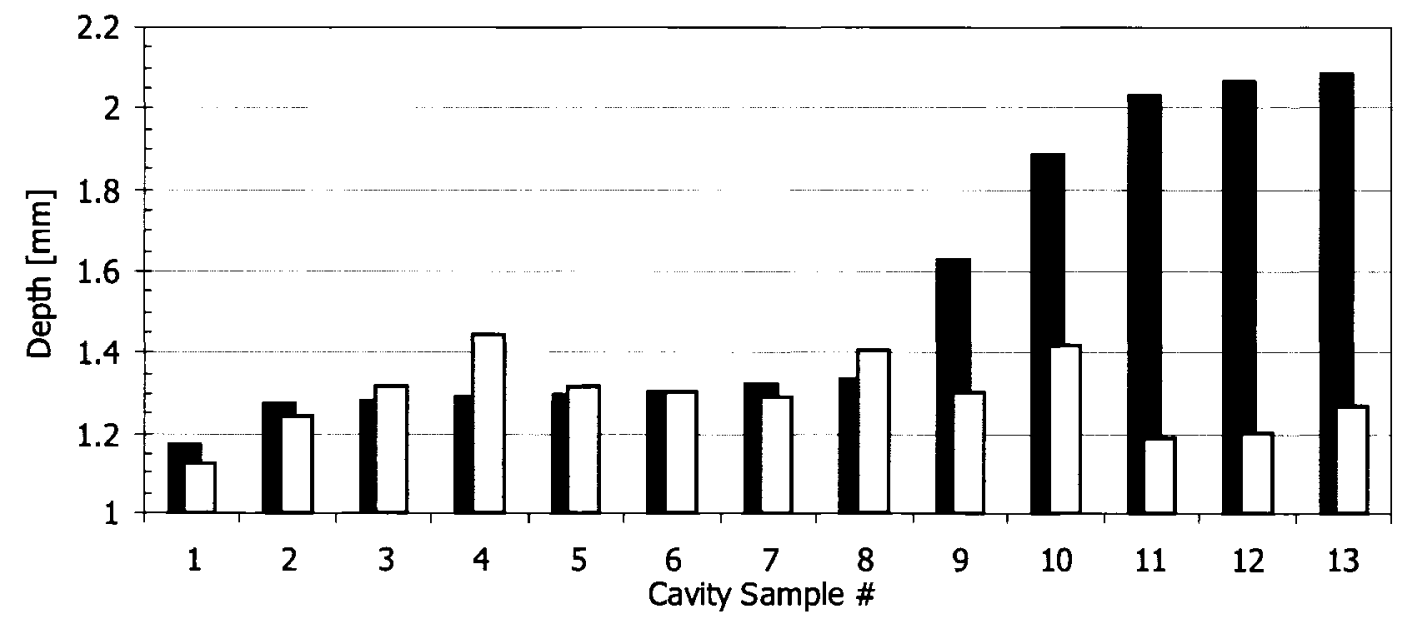

DAverage Theoretical Depth [mm] $\quad \square$ Measured Depth at Cavity Centre [mm]

Figure 4.7: Measured sizes of 13 etched cavity samples. 

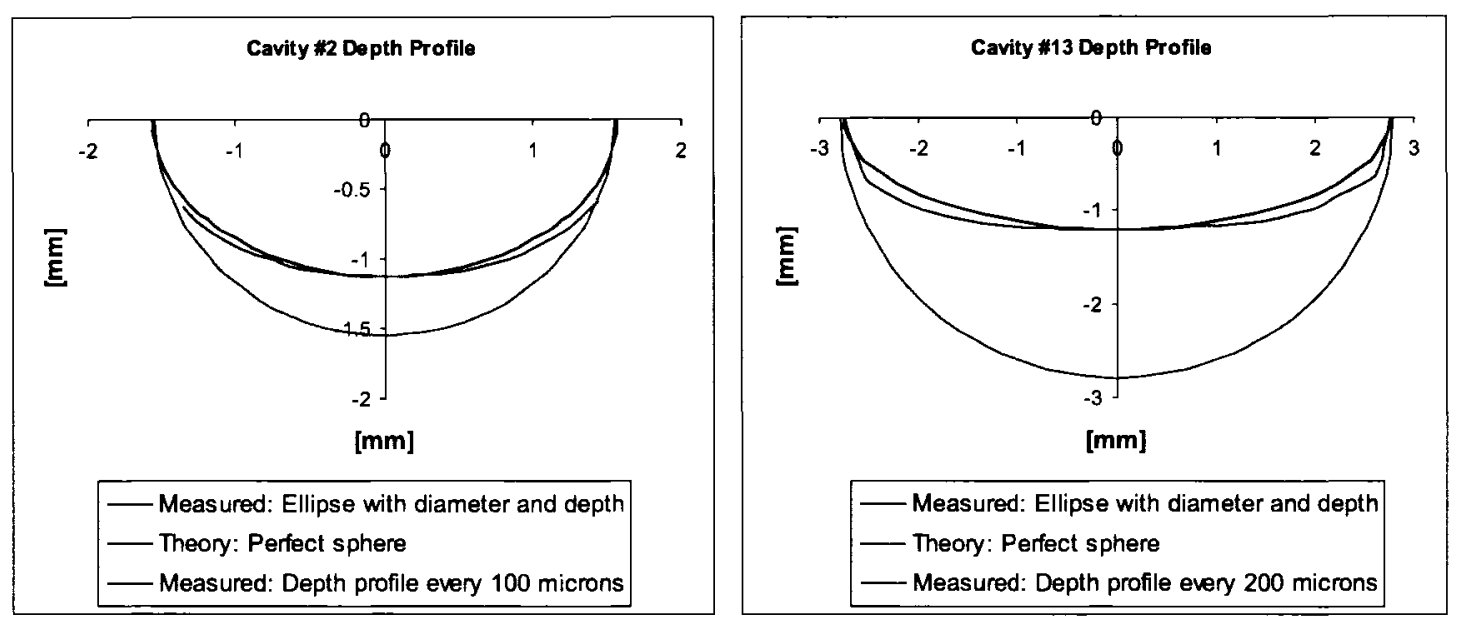

Figure 4.8: Measured elliptical profile for cavity 1 (left) and cavity 12 (right) of Figure 4.7.

using the radius and depth from Figure 4.7 as the major and minor radii. These results indicate that an elliptical cross-section results from the etching and the etched cavity is an oblate hemispheroid.

To further verify the etched cross-section, selected etched cavity samples were sawed in half, as shown in Figure 4.9 (left), and then visually inspected using a microscope, as shown in Figure 4.9 (right). To the naked eye, at least, the cross-section is elliptical. Scanning electron microscope (SEM) pictures of sectioned cavities, shown in Figure 4.10, also confirm a generally elliptical cross-section. Interestingly, close-up views of the sides, also included in Figure 4.10, show a slight aberration in the cross-section at the top of the cavity which curves inward slightly on both sides.

The cavity cross-sections were also used to further verify the surface roughness conclusions from Figure 4.5. The SEM pictures of the sectioned cavities in Figure 4.10 prove that, indeed, $70^{\circ} \mathrm{C}$ samples are noticeably smoother than those etched at $50^{\circ} \mathrm{C}$.

Based on the experimental results it was determined that cavities with a radius of $1.7004 \mathrm{~mm}$ can be micromachined in one self-limited etch using a fresh HNA having $\mathrm{HF}: \mathrm{HNO}_{3}: \mathrm{CH}_{3} \mathrm{COOH}=2: 3: 3$ for $1: 45 \mathrm{hrs}$ at $72^{\circ} \mathrm{C}$ with no agitation and laid 


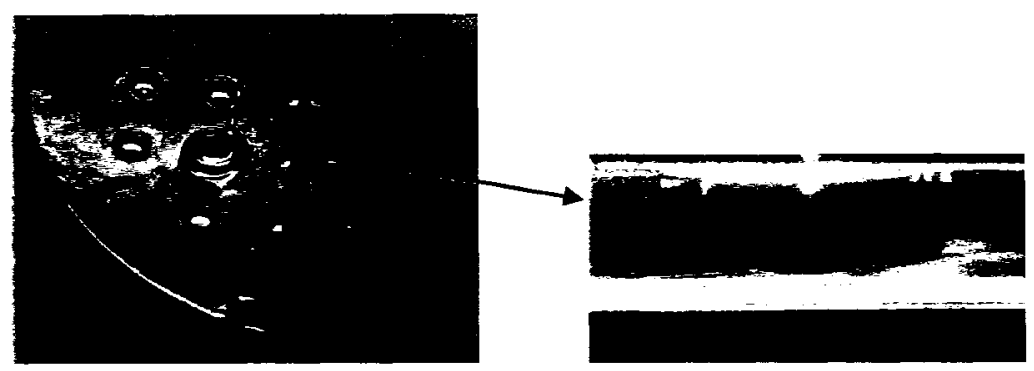

Figure 4.9: Sawed cavities (left) for inspecting the cross-section (right).
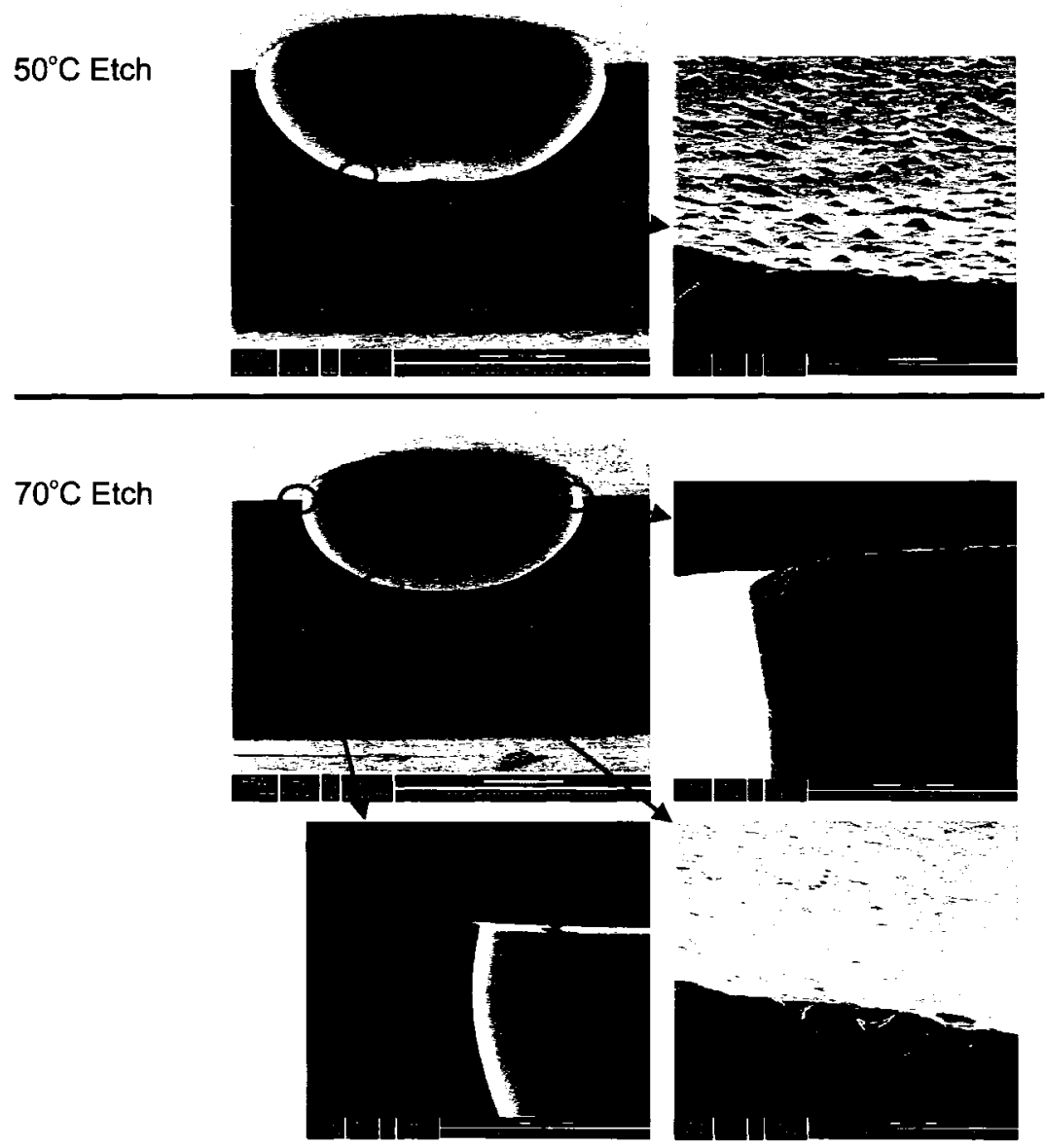

Figure 4.10: Scanning electron microscope pictures of sawed cavity cross-sections that were etched at $50^{\circ} \mathrm{C}$ and $70^{\circ} \mathrm{C}$. 


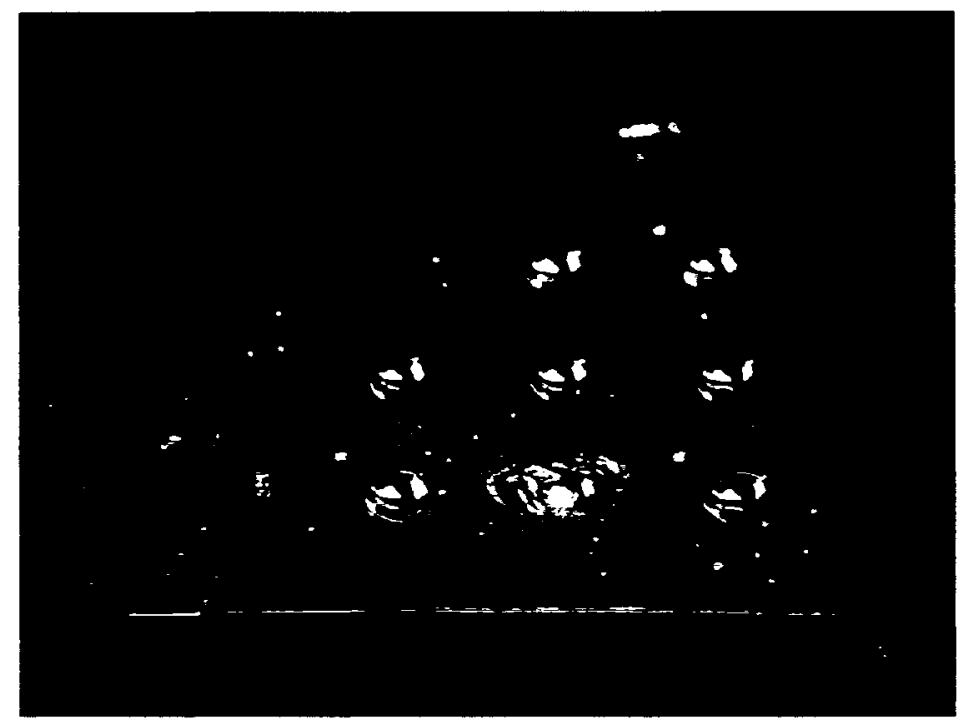

Figure 4.11: Final smooth and well-shaped oblate hemispherical cavities micromachined using self-limited isotropic etching.

flat. The expected cavity depth is about $1.2 \mathrm{~mm}$. For the etching of this sized cavity to be self-limited, the etch mask opening is $1.667 \mathrm{~mm}$ in diameter. The full self-limited isotropic etching procedure is given in Appendix A and this resulted in the final smooth and well-shaped micromachined cavities pictured in Figure 4.11 (before sputtering them with gold).

In addition to the final etch procedure, the isotropic etching experiments resulted in the following observations:

- The tape mask must be freshly applied without any gaps or cracks and the wafer can not be removed from the etchant for the etching to be self-limiting.

- Agitation does not improve the etch although increasing the etching temperature to $70^{\circ} \mathrm{C}$ results in a mirror smooth surface so that the polish step is not required.

- The etching is indeed self-limiting with the etched lateral radius being predicted by self-limited etching theory to within $9 \%$ and the etched depth being predicted by 


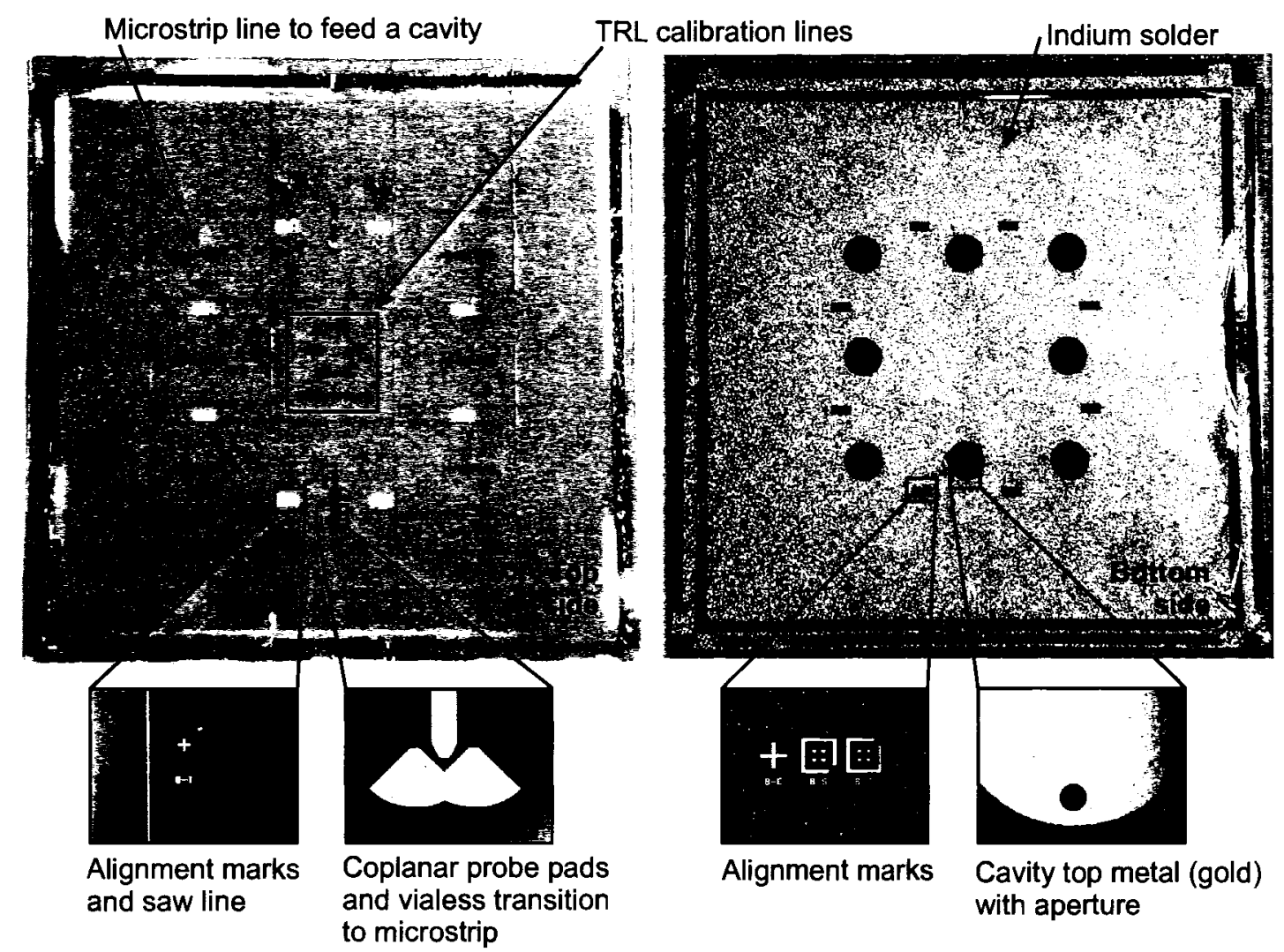

Figure 4.12: Final feed substrate.

the average theoretical depth (for cavities with a radius of less than about $2 \mathrm{~mm}$ ).

- The etched cavities are oblate hemispheroids with an elliptical cross-section (eccentricity related to cavity size) although the top edge of the etched cavities curve inward slightly.

\subsection{The Feed Substrate}

The feed substrate, shown in Figure 4.12, is placed on top of the etched silicon wafer and includes a number of microstrip transmission lines with ground-plane coupling apertures that "feed" electromagnetic energy to the cavities. This ground plane on the bottom 
side of the feed substrate is also the top metal of the cavities. The feed substrate also includes coplanar probe pads, so that the cavity performance can be measured using a wafer prober, and there is also a transition included from the coplanar probe pads to the microstrip feed lines. Finally, calibration structures are included to remove the effects of this transition from the measurements.

The feed substrate can be any suitable microwave material and alumina was arbitrarily chosen. Specifically, 5 mil thick $\left(h_{\mu s t r i p}=127 \mu \mathrm{m}\right)$ Superstrate TPS Alumina from Coorstek was selected since it has a lower loss $\operatorname{tangent}(\tan \delta=0.0001)$ and less variable dielectric constant $\left(\epsilon_{r}=9.9\right)$ at millimetre wave frequencies compared to other types of alumina from Coorstek. A $Z_{o}=50 \Omega, 1 \mu \mathrm{m}$ thick gold microstrip line on this substrate has a width of $w_{\mu s t r i p}=129 \mu \mathrm{m}$ at $77 \mathrm{GHz}$ (calculated using Agilent LineCalc software).

A single alumina substrate includes eight microstrip feed lines with corresponding apertures in the ground plane on the other side of the substrate so that a single alumina wafer can be used to test eight micromachined cavities. These microstrip lines on the top side of the alumina must be correctly aligned with the corresponding apertures in the ground plane on the bottom side. This front-to-back alignment is accomplished by patterning alignment marks on both sides of the alumina. First, gold is sputtered on the back side and then the alignment marks are etched according to a photolithographically defined pattern. A photoresist layer defining the top side microstrip is then aligned to the back side alignment marks by looking through the alumina using an infrared lamp. Gold is then evaporated on the front and patterned using lift-off of the front side photoresist and hence the front side microstrip is aligned to the back side apertures. Alignment using this procedure can repeatedly achieve front-to-back alignment to within $\pm 5 \mu \mathrm{m}$ in both the $x$ and $y$ directions. Since the back side metal is patterned first, all of the subsequent steps requiring alignment (top side metal, indium solder layer and bonding the silicon to alumina) are aligned to the back-side metal alignment marks. 
Each microstrip line also includes an open-circuit $\frac{3 \lambda_{g}}{4}$ stub which transforms the open circuit at its end to a short circuit at the centre of the aperture. This maximizes the magnetic field and hence also the magnetic coupling through the aperture meaning that the aperture can be made as small as possible for a desired amount of coupling. The stub length is $\frac{3 \lambda_{g}}{4}$ rather than $\frac{\lambda_{g}}{4}$ to ensure that the aperture is far from any end effects that could affect the coupling [132]. The physical length of the $\frac{3 \lambda_{g}}{4}$ stub is $1.086 \mathrm{~mm}$ past the aperture (also calculated using Agilent's LineCalc software).

Each alumina wafer includes four calibration structures (3 TRL structures and 1 verification line) to de-embed the effects of the probe pads from measurements. The TRL calibration plane is $800 \mu \mathrm{m}$ in from the probe pad, the standards are centred at $77 \mathrm{GHz}$ and they can be used to calibrate from 20 to $115 \mathrm{GHz}$.

Early iterations of the gold ground plane (on the back side of the feed substrate) was evaporated to achieve a smooth surface and hence low cavity loss. Unfortunately the evaporated layer contained small holes due to the underlying roughness of the alumina and even sputtering gold in later iterations resulted in some holes in the bottom gold layer. Although inconsequential for a normal microstrip ground plane, when the back side metal is used as a cavity wall these holes may cause electromagnetic energy leakage from the cavity increasing the loss and decreasing the quality factor.

The final alumina processing sequence was modified to prevent holes in the bottom gold layer by using thicker gold $(2 \mu \mathrm{m})$, thicker photoresist and soft-contact lithographic alignment. The early iteration holes in the gold ground plane were likely caused during the gold-etch that is used to pattern the bottom gold layer. After gold was sputtered everywhere on the back side, the gold was etched while the photoresist protected the areas where gold was desired. If there were holes in the photoresist, then gold was exposed to etchant where it shouldn't be and thus holes in the gold were created. The initial photoresist thickness was not sufficient to fully cover the roughness of the alumina and 
thus thicker photoresist was needed. Furthermore, initially the photomask was pressed against the photoresist layer on the alumina, a procedure known as "hard-contact" alignment, so that the photomask contacts the alumina everywhere. Since the photomask is flat, the hard-contact pressure will tend to flatten the top side of the photoresist layer by "spreading" the photoresist. More spreading occurs on the peaks of the alumina surface (since more pressure is exerted on the peaks of the alumina surface than on the valleys) and the photoresist spreading may expose the gold to the etchant where it shouldn't be exposed resulting in undesired holes in the gold. Using "soft-contact" photomask alignment alleviates this problem since the mask is very close but not touching the photoresist and hence the photoresist is not spread and holes do not form. When soft-contact alignment was used in later fabrication iterations, along with thicker photoresist, there were no holes near the cavities.

\subsection{Vialess Probe Pads}

Cavity measurements are made with a wafer prober by placing coplanar ground-signalground (GSG) probes on coplanar probe pads located on the feed substrate. The cavity feeds, however, are microstrip lines, therefore a transition from the coplanar probes pads to microstrip is needed. Initially this transition was designed using vias, as shown in Figure 4.13, to connect the coplanar ground pads on the top side of the alumina to the microstrip ground plane on the bottom side. The smallest vias that can be drilled at CRC were 8-12 mils in diameter $(203.2-304.8 \mu \mathrm{m})$ and these were drilled through large gold pads on the top side of the alumina using an ultrasonic impact grinder. These large gold pads must be at least 3 times larger than the drilled vias to allow for drill placement variation and still maintain metal contact at the periphery of the drilled via hole (for a continuous metal connection from the top of the alumina to the ground plane on the 


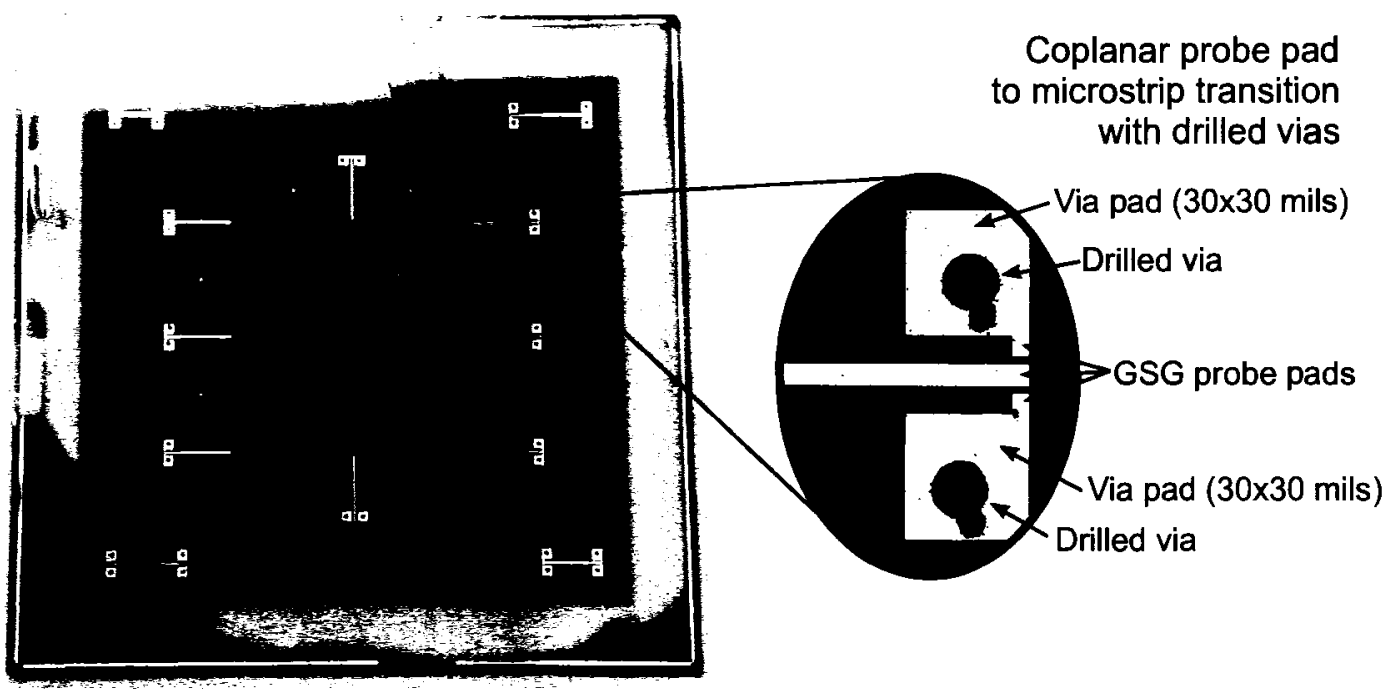

Figure 4.13: Top side of an early alumina feed substrate using drilled vias for the coplanar probe pad to microstrip transition.

bottom) and are therefore about $30 \times 30 \mathrm{mils}(762 \times 762 \mu \mathrm{m})$.

Although this via transition was included on the fabricated alumina substrate shown in Figure 4.13, it was later determined that these vias and via pads cannot be used at $77 \mathrm{GHz}$. Even though the 8-12 mil diameter vias are the smallest diameter vias possible at CRC this size is intended for circuits operating under $30 \mathrm{GHz}$ and not for W-band. For circuits operating above $30 \mathrm{GHz}, 30 \times 30$ mils via pads are large enough to support undesired modes of propagation that reduce the effectiveness of the transition. This was confirmed using method of moments simulation (Agilent's Momentum software), which is shown in 4.14. Further simulations indicate that smaller via pads can operate up to Wband (for example a $120 \times 120 \mu \mathrm{m}$ via pad in a typical commercial MMIC process could be used where the vias are made using dry etching of a GaAs substrate), however, CRC does not have the capability to drill vias smaller than 10 mils in diameter. Furthermore, even if smaller vias (and via pads) were possible, drilling vias is time consuming and may scratch the back side of the alumina which could increase the metal loss in the cavity 


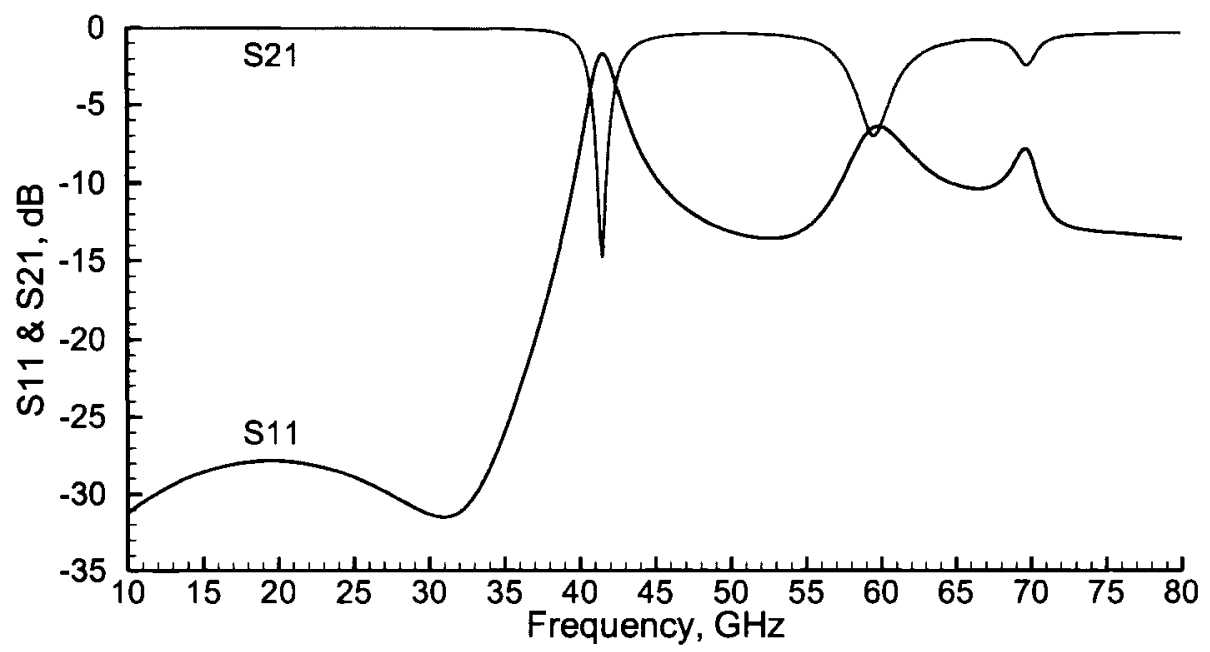

Figure 4.14: Method of moments simulation showing that the ground-signal-ground probe pad to microstrip transition, using drilled vias, only works up to about $30 \mathrm{GHz}$.

and thus lower the quality factor. Finally, the via walls must be metalized so that the via holes are connected to both the via pads and the bottom side ground plane and this requires extra and more complicated processing steps.

Given these difficulties, a vialess transition from the GSG probe pad to microstrip was used. A test alumina substrate with a variety of vialess transitions was designed and fabricated, a photograph of which is shown in Figure 4.15. During measurements, the ground-signal-ground probe is placed with the centre probe on the microstrip line and each ground probe on the quarter-wave stubs that are adjacent to the microstrip line. The ground probes each contact near one end of a quarter-wave stub with an open at the other end of the stub. The stub transforms that open circuit to a short circuit in the vicinity of the ground probe and the potential of the ground probes is passed to the microstrip ground plane on the other side of the wafer, without using a via, at the design frequency where the stub is a quarter-wavelength long. At the design frequency these vialess transitions are better than transitions with vias since there is no inductance or 

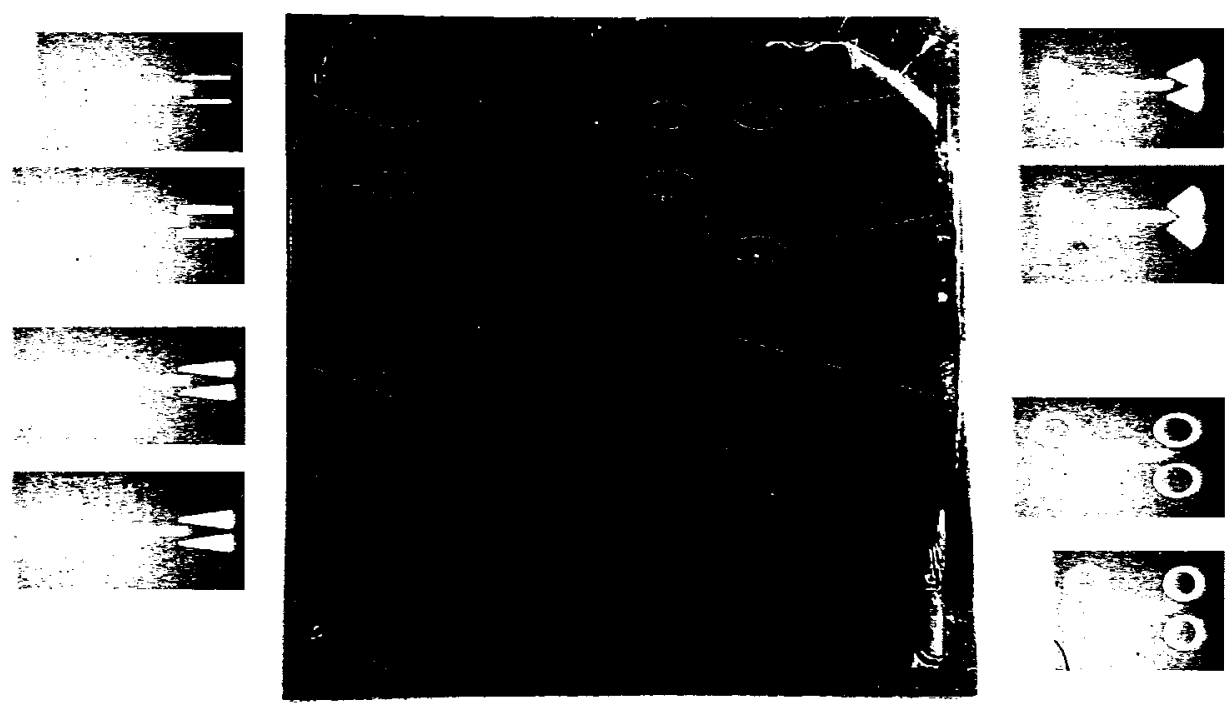

Figure 4.15: Test substrate of a number of different vialess transitions from groundsignal-ground probe pads to microstrip.

any other parasitics contributed by the vias.

The bandwidth of each of these transitions increases as the characteristic impedance of the stubs decreases since, at the location of the ground probe, the impedance between a stub and the ground plane, $Z_{i n}=-j Z_{o} \cot \beta l$, varies less with frequency for lower stub $Z_{o}$. Therefore, the test wafer of Figure 4.15 includes quarter-wave stubs with decreasing characteristic impedances: first two straight stubs (50 $\mu \mathrm{m}$ and $100 \mu \mathrm{m}$ wide) and then a series of radial stubs (with angles $20^{\circ}, 25^{\circ}, 60^{\circ}$ and $90^{\circ}$ ). The last two radial stubs are "merged radial stubs" because at $60^{\circ}$ and $90^{\circ}$ the ends of the stubs are merged together since the separation between the stubs is fixed by the probe pitch and cannot be changed. Both ends of the stubs are open-circuits so it doesn't matter if they're merged and in any event both ground probes of the GSG probe are already connected together in the probe itself.

The test substrate in Figure 4.15 also includes ring resonators that force a short-circuit near the ground probes since the circumference is a half-wavelength. Measurements of 
these structures confirm that they can be used successfully as a vialess transition over a wide bandwidth, however, accurately simulating them is difficult. The rings have no straight edges to place simulation ports (unlike the straight and radial stubs), hence the ports must be placed inside the rings (known as "internal ports"). The simulated results are very sensitive to the location of the internal ports therefore the comparison of the prototype transitions was limited to the straight and radial stubs that can be more reliably simulated.

The straight and radial stub vialess transitions shown in Figure 4.15 must be fabricated in back-to-back configurations for measurement with ground-signal-ground coplanar probes, however, single transitions can be simulated in Momentum using a groundsignal-ground port at the transition followed by a very short length of microstrip and a microstrip port. This allows a more direct comparison of the different transitions, assuming the simulations are accurate, as it does not include the effects of the line separating the back-to-back transitions (with extra loss and nulls produced when this separation line is a quarter-wavelength). Momentum simulations of single transitions were determined to be accurate since measurements of the back-to-back transitions were found to be very well correlated with corresponding Momentum simulations for all of the types of back-to-back transitions, examples of which are shown in Figures 4.16, 4.17 and 4.18.

Therefore, the simulations of single transitions are assumed to be reasonably accurate for comparing and selecting a vialess transition to use in the final feed substrate. Results of single transition simulations are shown in Table 4.2 and, as expected, the $90^{\circ}$ merged radial stubs have the largest bandwidth since the stubs have the lowest characteristic impedance.

The $90^{\circ}$ merged radial stubs were therefore chosen to replace the drilled vias in the final cavity feed substrate shown in Figure 4.12. The length of the stubs was selected using Momentum simulations for a centre frequency of around $77 \mathrm{GHz}$. A simulation of 


\begin{tabular}{|l|c|c|c|c|c|c|c|}
\hline Stub type & Centre & \multicolumn{3}{|c|}{ BW where $\mathrm{S}_{11}<-15 \mathrm{~dB}$} & \multicolumn{2}{c|}{$\mathrm{S}_{21}$ over BW in dB } \\
\cline { 3 - 8 } & Freq. & Min. & Max. & $\mathrm{BW}$ & $\% \mathrm{BW}$ & Min. & Max. \\
\hline \hline Straight, $50 \mu \mathrm{m}$ wide & 70.42 & 52.71 & 83.61 & 30.90 & $\mathbf{4 3 . 8 8 \%}$ & -0.117 & -0.363 \\
Straight, $100 \mu \mathrm{m}$ wide & 69.03 & 46.81 & 83.26 & 36.45 & $\mathbf{5 2 . 8 0 \%}$ & -0.108 & -0.403 \\
Radial, 20 $0^{\circ}$ wide & 60.69 & 39.17 & 77.01 & 37.84 & $\mathbf{6 2 . 3 5 \%}$ & -0.084 & -0.332 \\
Radial, 25 ${ }^{\circ}$ wide & 59.48 & 37.08 & 76.67 & 39.59 & $\mathbf{6 6 . 5 6 \%}$ & -0.080 & -0.334 \\
${\text { Merged Radial, } 60^{\circ} \text { wide }}$ & 68.33 & 40.21 & 93.33 & 53.12 & $\mathbf{7 7 . 7 4 \%}$ & -0.088 & -0.397 \\
${\text { Merged Radial, } 90^{\circ} \text { wide }}$ & 69.38 & 37.08 & 94.72 & 57.64 & $\mathbf{8 3 . 0 8 \%}$ & -0.088 & -0.424 \\
\hline
\end{tabular}

Table 4.2: Single transition bandwidth (BW) for the vialess ground-signal-ground probe pad to microstrip transitions in Figure 4.15. All frequencies in GHz.

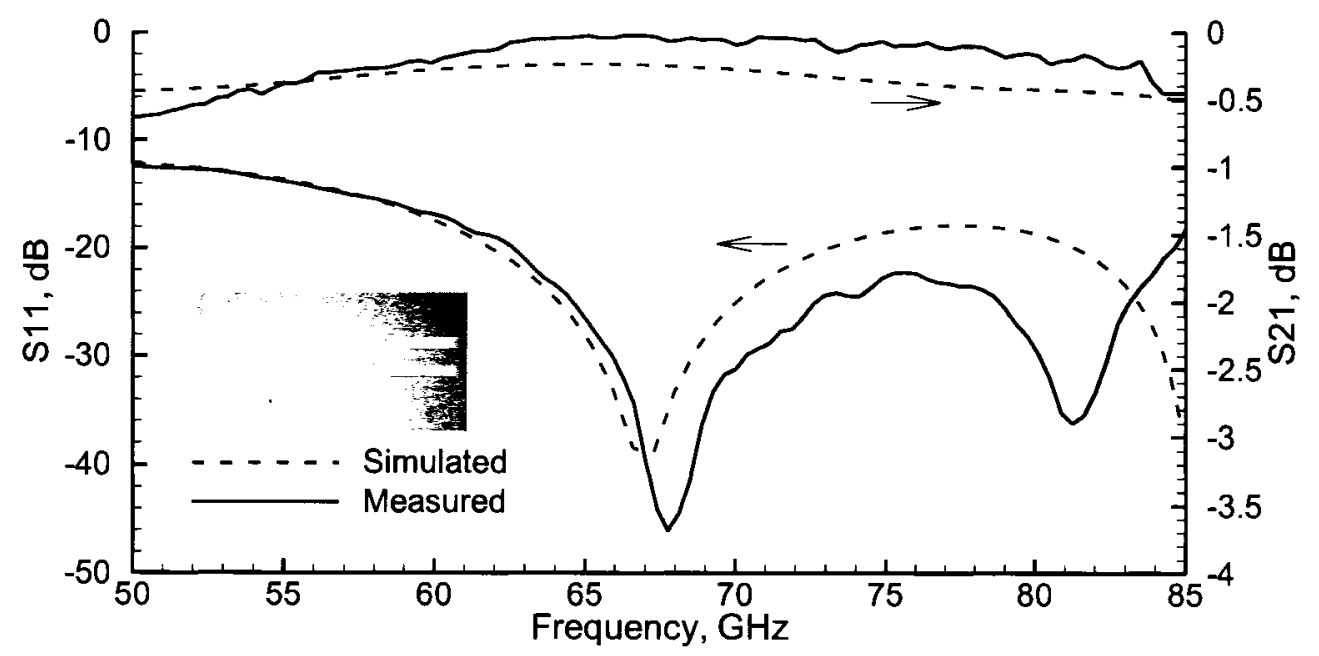

Figure 4.16: Measurements and simulations of straight stub vialess probe pads from the vialess test wafer in Figure 4.15. 


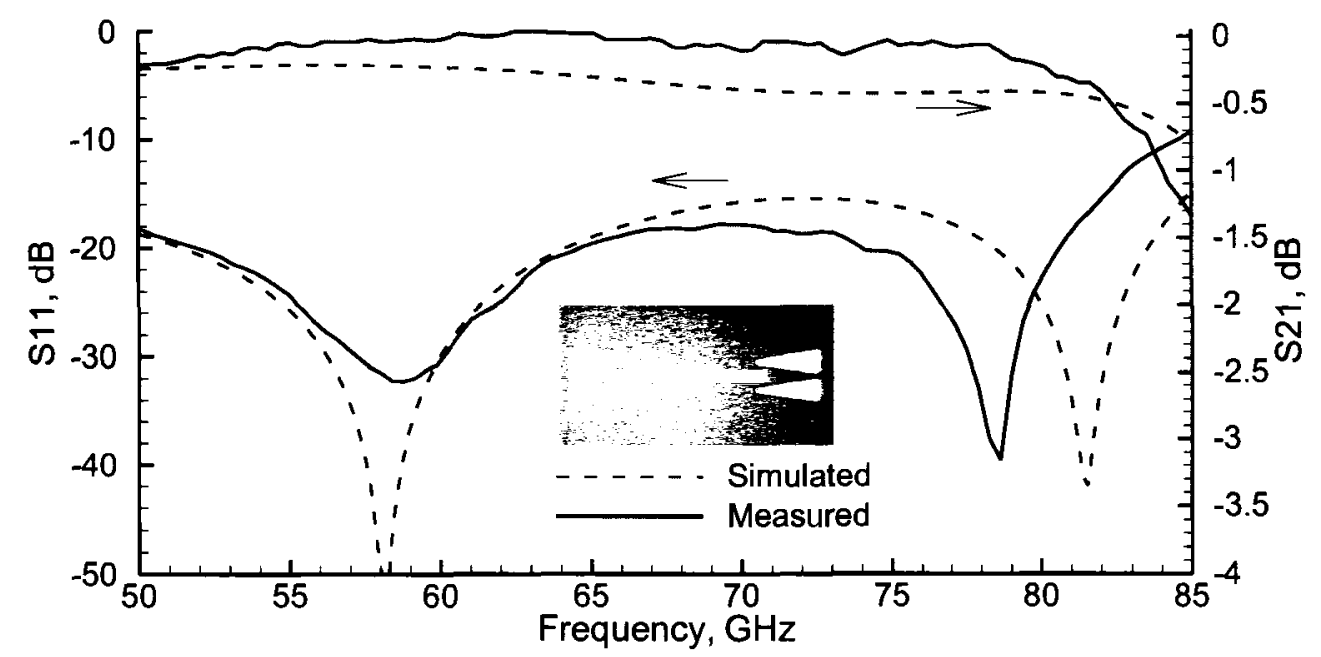

Figure 4.17: Measurements and simulations of radial stub vialess probe pads from the vialess test wafer in Figure 4.15.

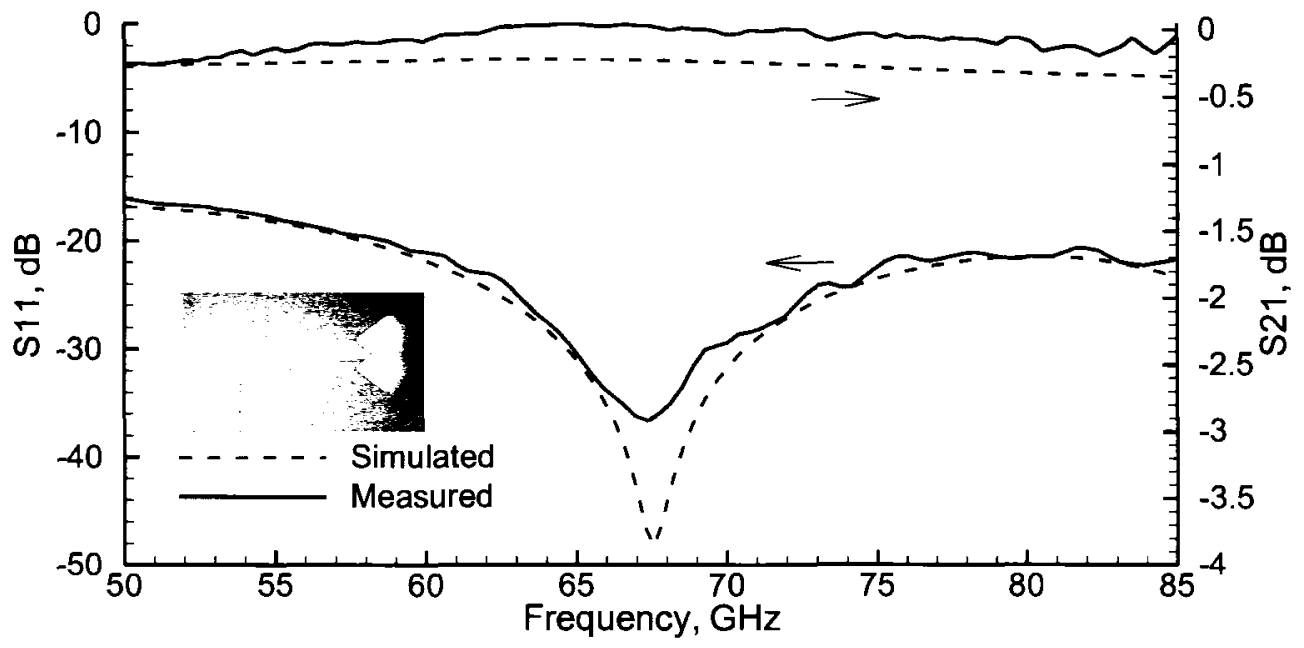

Figure 4.18: Measurements and simulations of merged radial stub vialess probe pads from the vialess test wafer in Figure 4.15. 


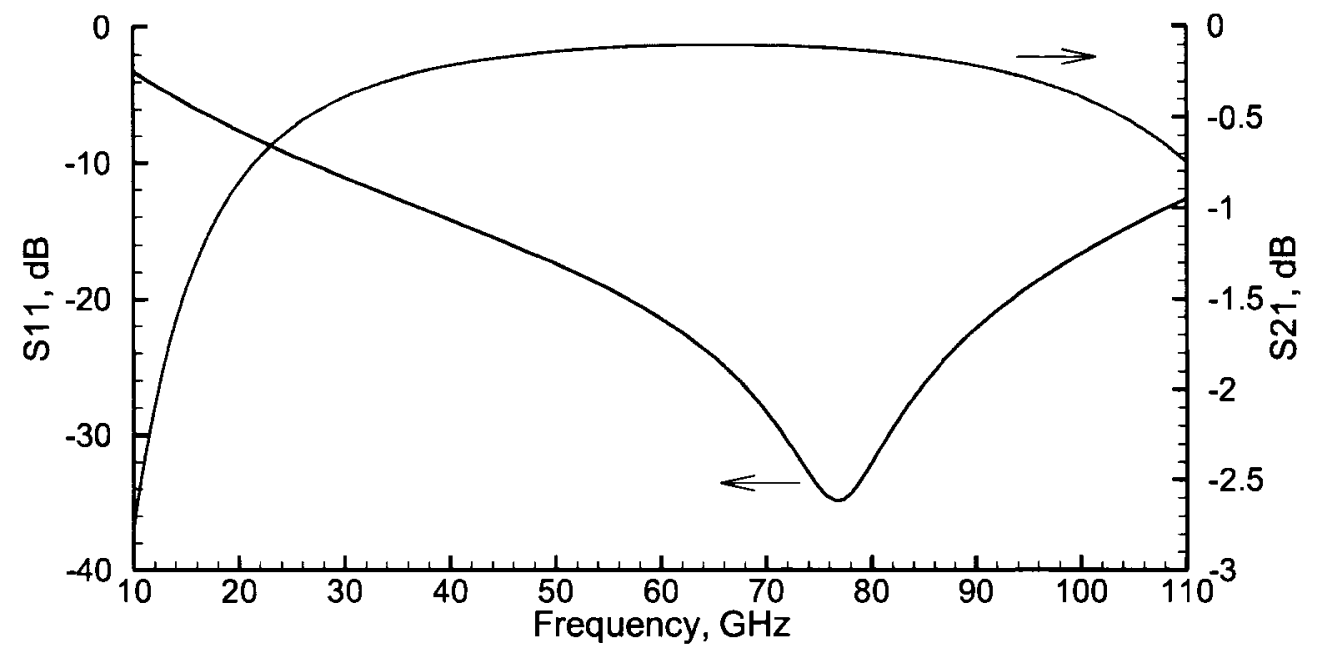

Figure 4.19: Simulation of a single $77 \mathrm{GHz}$ vialess probe pad designed for micromachined cavity measurements ( $90^{\circ}$ merged radial stubs, see Figure 4.12 ).

the final, single transition predicts a centre frequency of $76.67 \mathrm{GHz}$ and a $\mathrm{S}_{11}<-15 \mathrm{~dB}$ from $43 \mathrm{GHz}$ to at least $85 \mathrm{GHz}$ with a maximum $\mathrm{S}_{21}$ of $-0.17 \mathrm{~dB}$ over that range. These simulated results are shown in Figure 4.19 and they compare favourably to existing vialess GSG probe pad to microstrip transitions $[168,169]$.

This $77 \mathrm{GHz}$ vialess probe pad transition was measured in a back-to-back transition test. The TRL calibration structures included on the final cavity feed substrate (see Figure 4.12) includes a $5.45 \mathrm{~mm}$ long verification line (the bottom and longest of the TRL structures). Measuring this structure with the system calibrated to the probe tips using open-short-load (OSL) calibration (with a standard calibration substrate), tests the transition by itself (albeit in back-to-back configuration). Figure 4.20 shows this measured response along with a Momentum simulation of the verification line with ports at the probe tips.

The verification line was measured again with TRL calibration thereby allowing the $77 \mathrm{GHz}$ vialess probe pad transition to be de-embedded leaving a simple measurement 


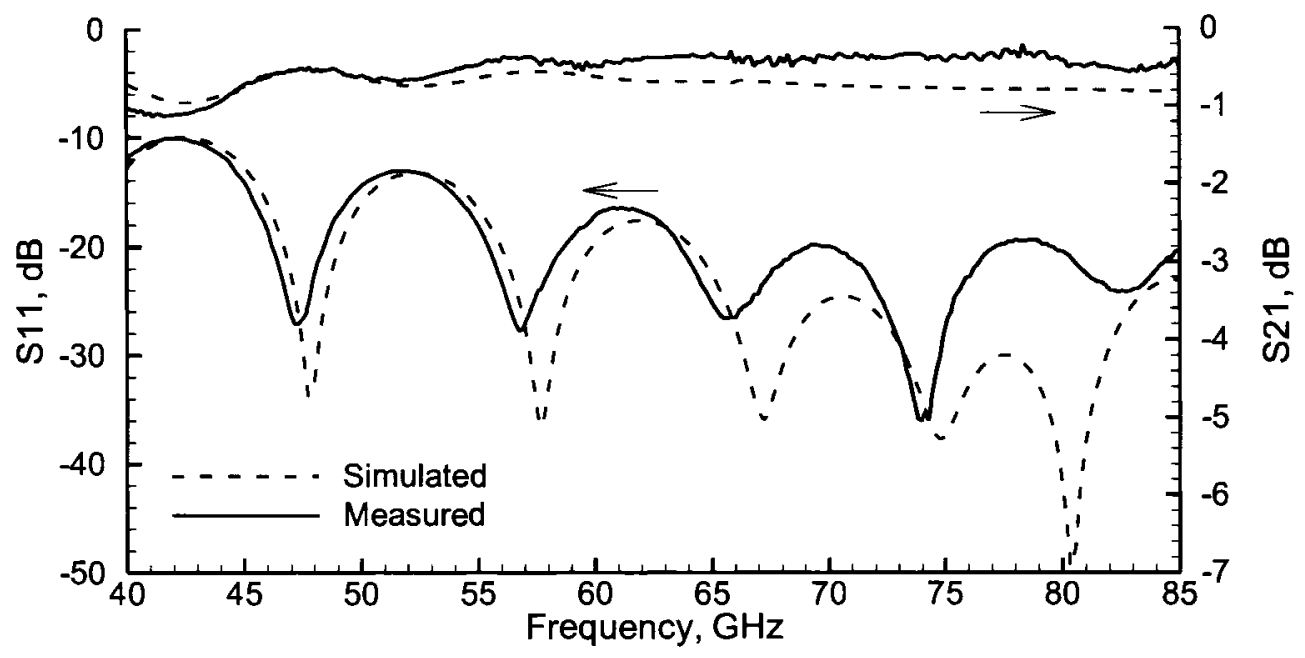

Figure 4.20: Measurements and simulations of the TRL verification line with OSL calibration at the probe tips to test the back-to-back vialess transition used for cavity measurements.

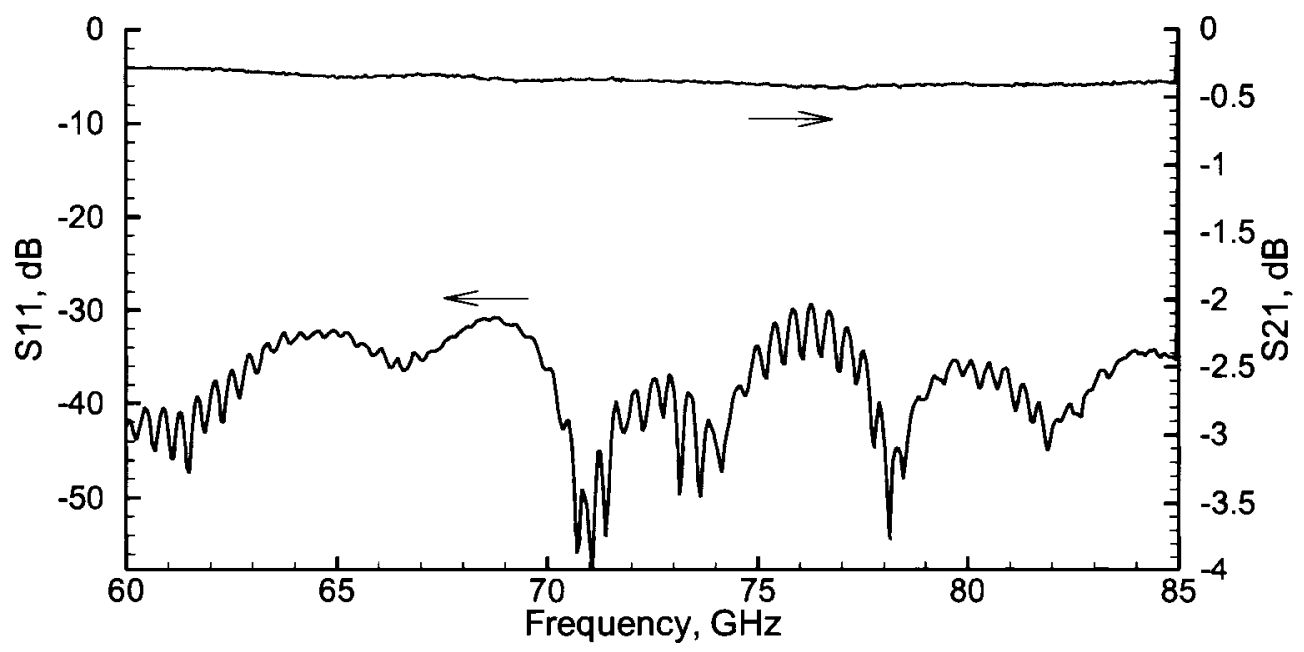

Figure 4.21: Measurement of $50 \Omega$ verification line on final cavity feed substrate after vialess probe pads are de-embedded using TRL calibration. 
of a $3.7 \mathrm{~mm} 50 \Omega$ line to verify the transition (and the calibration) function correctly. Figure 4.21 (on the previous page) shows the measured response of the verification line after the transitions are de-embedded using the TRL standards. The measured microstrip is well matched and low loss. This clearly shows the suitability of these vialess probe pads for cavity measurements from $60 \mathrm{up}$ to $85 \mathrm{GHz}$.

\subsection{Assembly}

Isotropic etching of a silicon wafer produces an open hemispherical micromachined cavity. In order to form a closed cavity resonator, this etched silicon wafer must be bonded to the feed substrate. Direct, anodic or intermediate layer bonds [170] can be used to bond these two wafers together without using epoxy or solder at temperatures below the melting point of the metalized cavity walls [171, 172]. These techniques, however, require specialized equipment that is not available at CRC. Using conductive silver epoxy to bond the two wafers will slightly distort the hemispherical shape of the cavity since the epoxy layer thickness is at least 2 mils thick $(50 \mu \mathrm{m})$ and may be up to $4-8$ mils thick. Furthermore, epoxy might flow into the cavity during the bonding process. Worse still is that the conductivity of the silver epoxy is only $1 / 166$ th of the conductivity of gold resulting in almost a $40 \%$ reduction in the loaded quality factor. Alternatively, a solder layer is thinner than epoxy and it will not flow where the bonding surface is not wetted and can therefore be prevented from flowing into the cavity. Perhaps more importantly the conductivity of tin, or better still indium, solder is much closer to that of gold resulting in much less impact on the loaded quality factor. Therefore, soldering was chosen to bond the alumina feed substrate to the silicon cavity wafers.

Tin was selected as the initial solder material to try since the CRC microfabrication team has the most experience with soldering using tin. First, a $2000 \AA$ layer of tin solder 


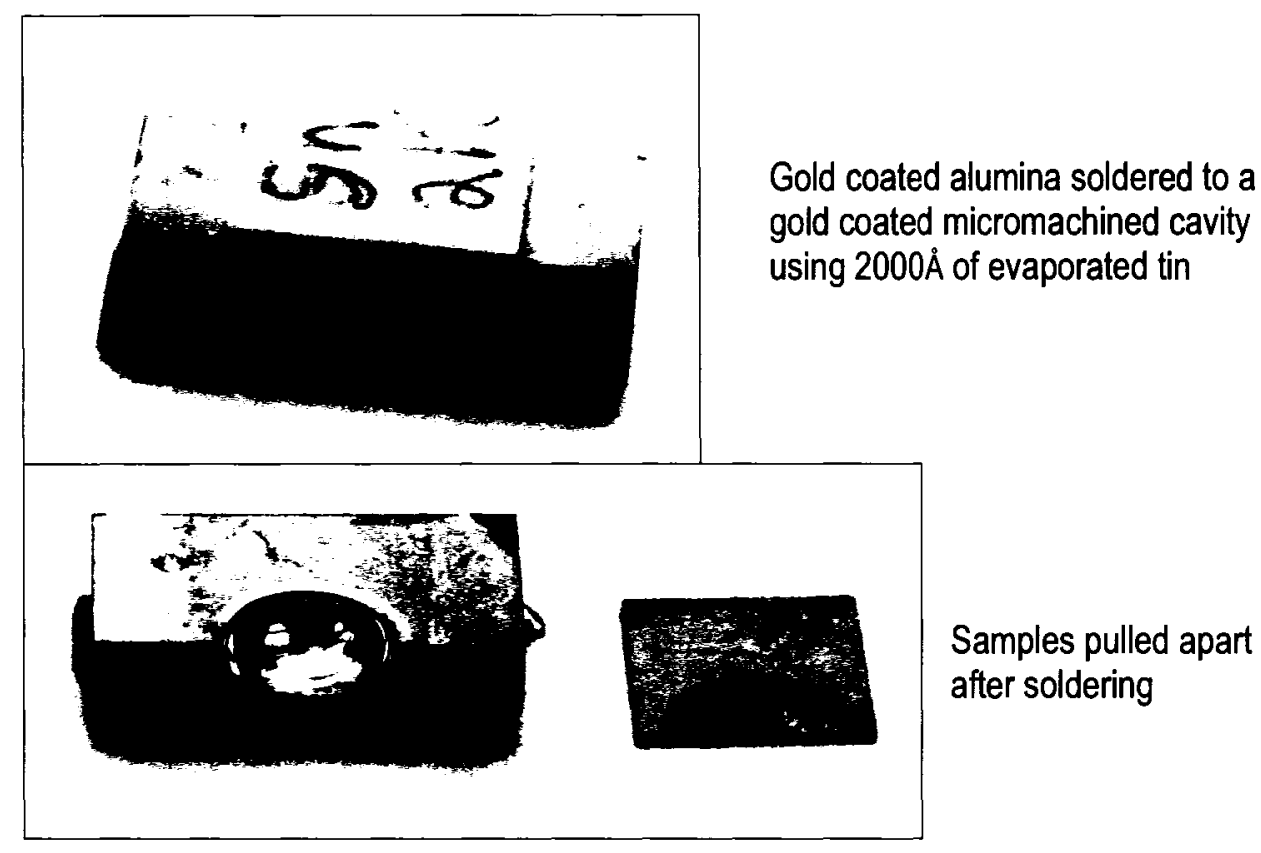

Figure 4.22: Gold-tin-gold soldering experiments.

was evaporated on a gold-coated piece of alumina and flux was then applied to the sample. The alumina piece was then contacted to a metalized silicon substrate having a previously micromachined cavity. The two pieces were then heated to reflow the solder and the resulting bonded cavity is shown in Figure 4.22. Another sample was soldered and then pulled apart to inspect the quality and strength of the bond and this is also shown in Figure 4.22. Although the solder did not appear to flow into the cavity, which is highly desirable, closer inspection of the alumina piece revealed that there is still flux on the surface and little evidence of metal to metal contact indicating that the flux may have been holding the pieces together. Varying thicknesses of tin $(2000 \AA$ to $8000 \AA)$ did not appear to fix the problem.

Similar experiments were performed with indium solder. Indium solder is better than tin because indium has a higher conductivity (almost as high as gold), a lower melting point and it can be used without flux. Indium was deposited onto a piece of alumina, 

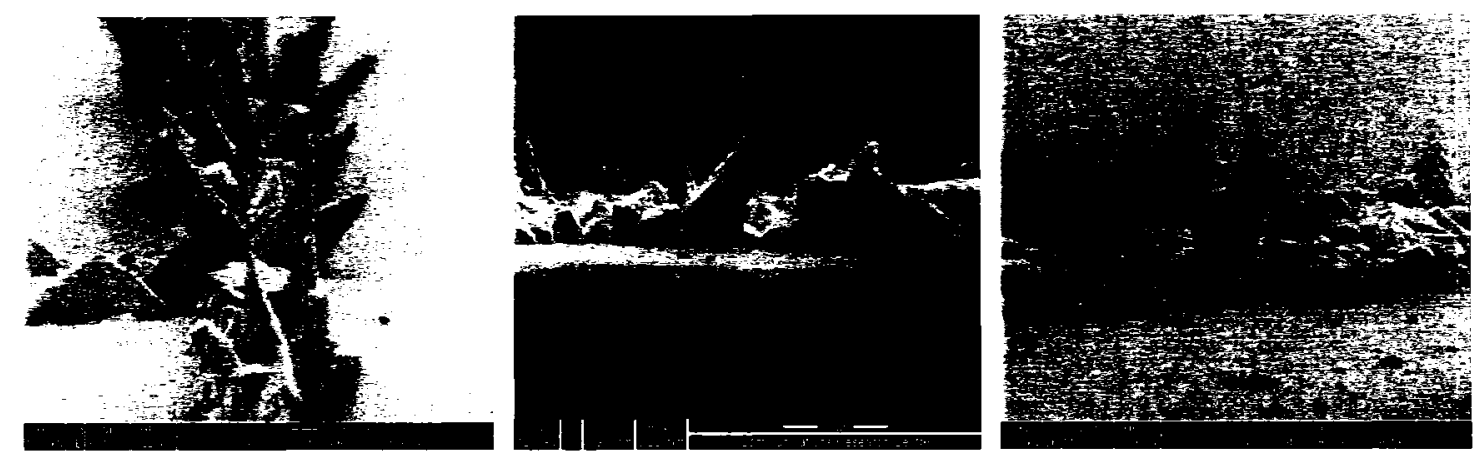

Figure 4.23: Results of initial indium plating development.
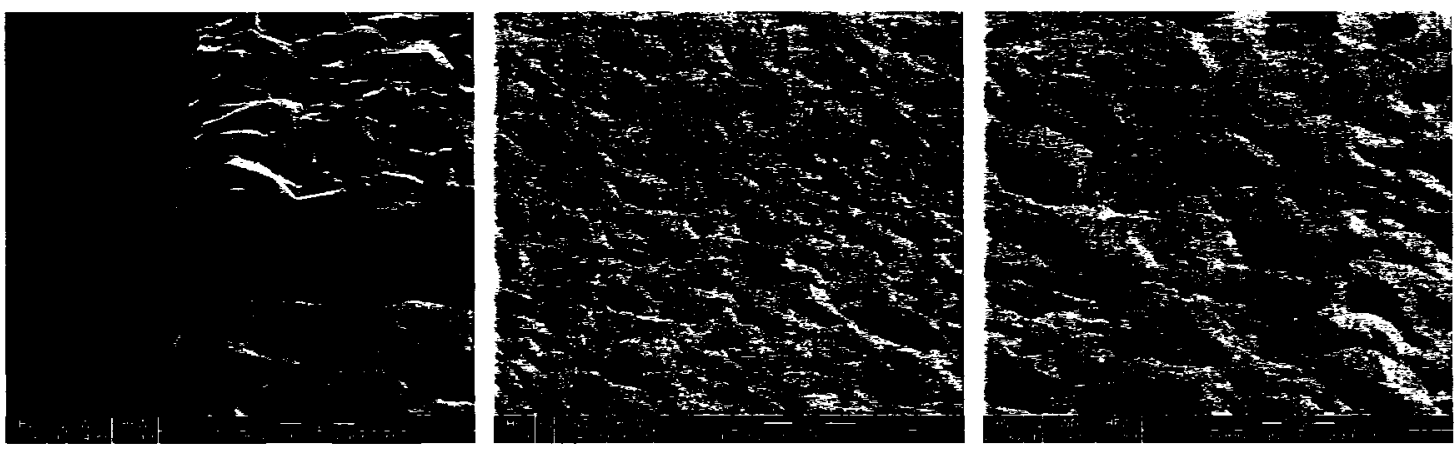

Figure 4.24: Results of final indium plating.

contacted with pressure to a previously metalized micromachined silicon cavity and then heated. After varying the indium thickness it was found that $30 \mu \mathrm{m}$ successfully bonded the alumina to the silicon. The strength of the bond was verified using repeated droptests where the solder layer held after many drops. Furthermore, the indium solder does not appear to flow into the cavity.

Next an indium plating process was developed to repeatably deposit a specific thickness of indium on an alumina wafer. Initial efforts resulted in big indium grains and spikes as shown in Figure 4.23, however, the process was eventually refined by the CRC microfabrication team to result in a uniform distribution with small grains and no spikes as shown in Figure 4.24. This smooth indium layer can be patterned as shown in Figure 4.25 to get a continuous circular and well-defined contour defining the solder bond 

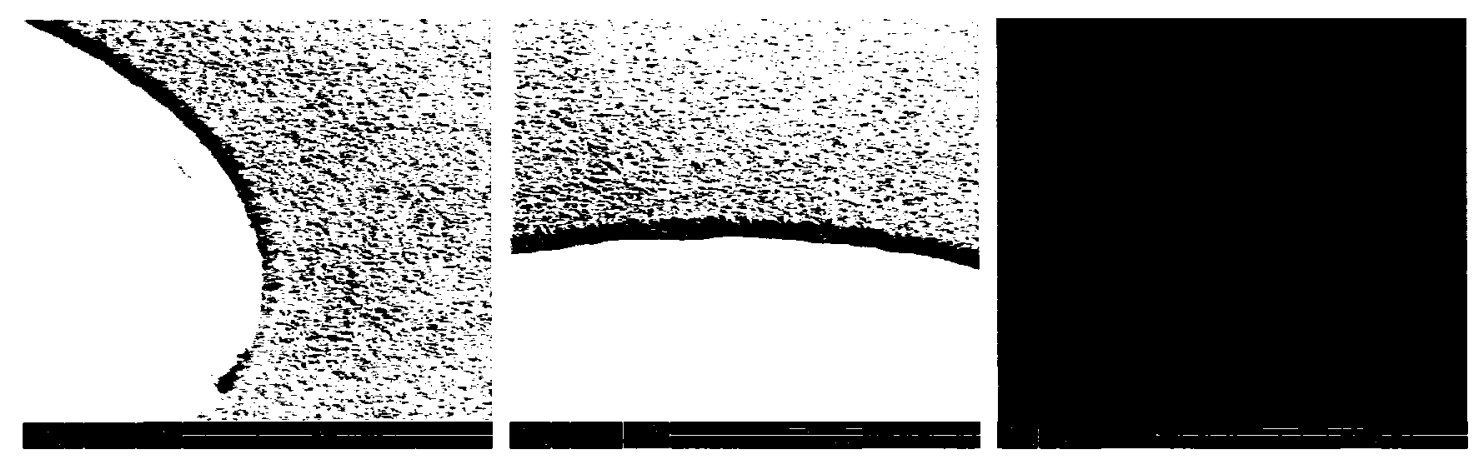

Figure 4.25: Results of patterned indium solder layer.

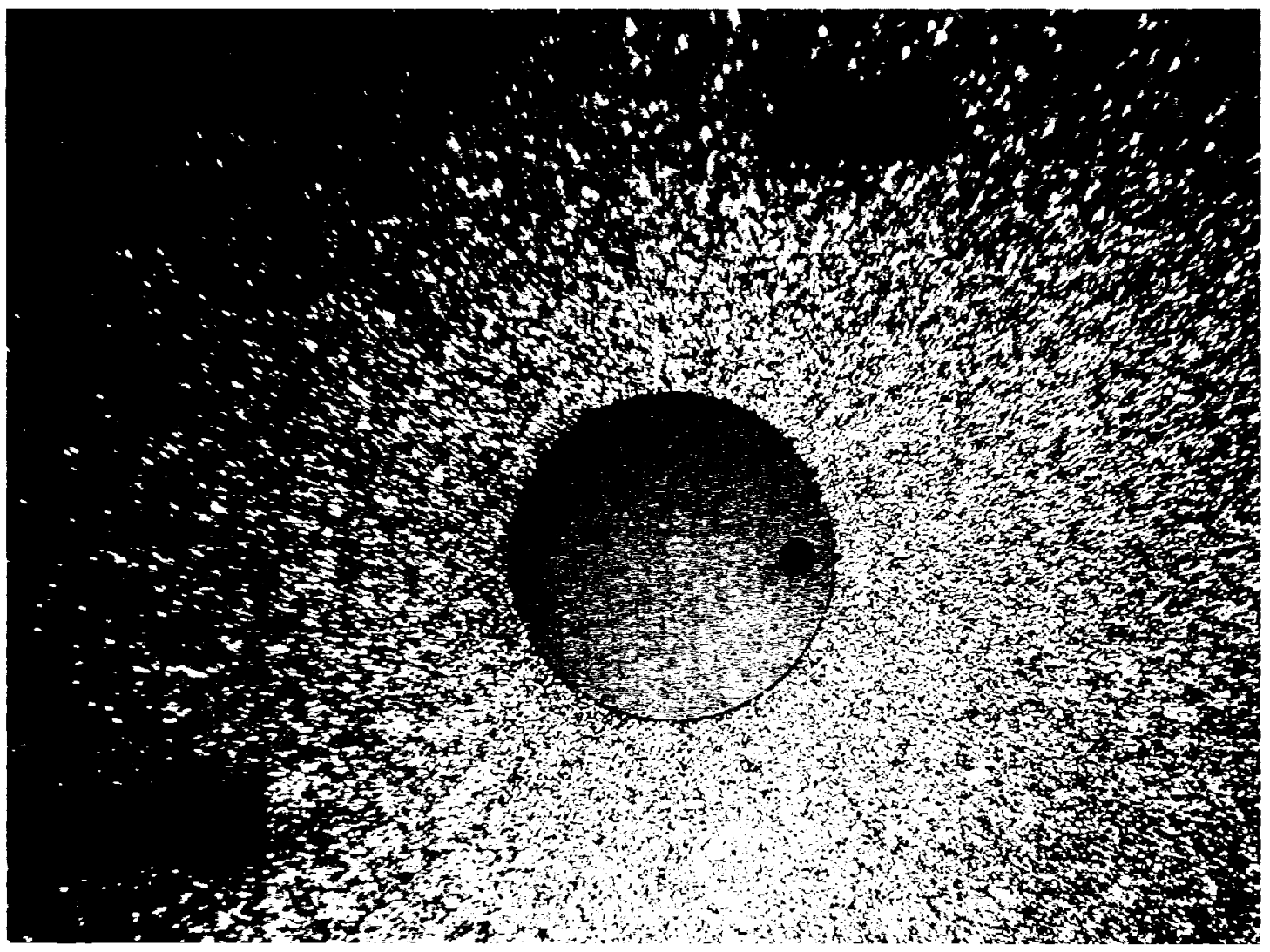

Figure 4.26: Exposed cavity top surface (gold microstrip ground plane), aperture and alignment marks after indium solder layer patterning. 
at the outer edge of the cavity, so that the top side of the cavity is the bottom side gold of the alumina and not the indium layer which has a lower conductivity. Patterning the indium also ensures that the aperture in the microstrip ground plane is not covered by the solder (the exposed aperture in the gold can also be seen in Figure 4.25). Additionally, the gold alignment marks on the bottom side of the alumina are also exposed by patterning the indium, as shown in Figure 4.26 (on the previous page).

The alumina with patterned indium is then soldered to the micromachined silicon wafer using a flip-chip bonder that can align the two wafers, bring them in contact, apply pressure and then heat the samples to reflow the solder. The first of these steps, alignment, ensures that the alumina substrate is precisely positioned on the silicon wafer so that the aperture is positioned at the point of maximum magnetic field inside the micromachined cavity. This placement ensures the smallest aperture for coupling energy in and out of the cavity, which simplifies the analysis in Section 3.4 (the "small aperture" assumption). The silicon wafer alignment marks are initially patterned in the tape, and the SiN mask, during reactive ion etching. Then they are covered using additional tape before the main HNA etch, otherwise the large amount of HNA entering directly through the opening in the initial tape mask would render them unrecognizable after the etch. Instead, only a very small amount of HNA reaches the alignment mark openings in the SiN by starting from the cavity tape mask openings and then traveling between the tape and the SiN layer. Therefore, only a small amount of HNA etches each small alignment mark in the silicon substrate. These HNA etched alignment marks are then aligned with corresponding gold alignment marks on the bottom side of the alumina (exposed by an opening in the indium), as was shown in Figure 4.26. It is estimated that the alignment error of this procedure is less than $75 \mu \mathrm{m}$.

Figure 4.27 shows a cross-section of an aligned and soldered sample. The indium solder did not flow into the cavity and there are no visible gaps in the solder bond. 


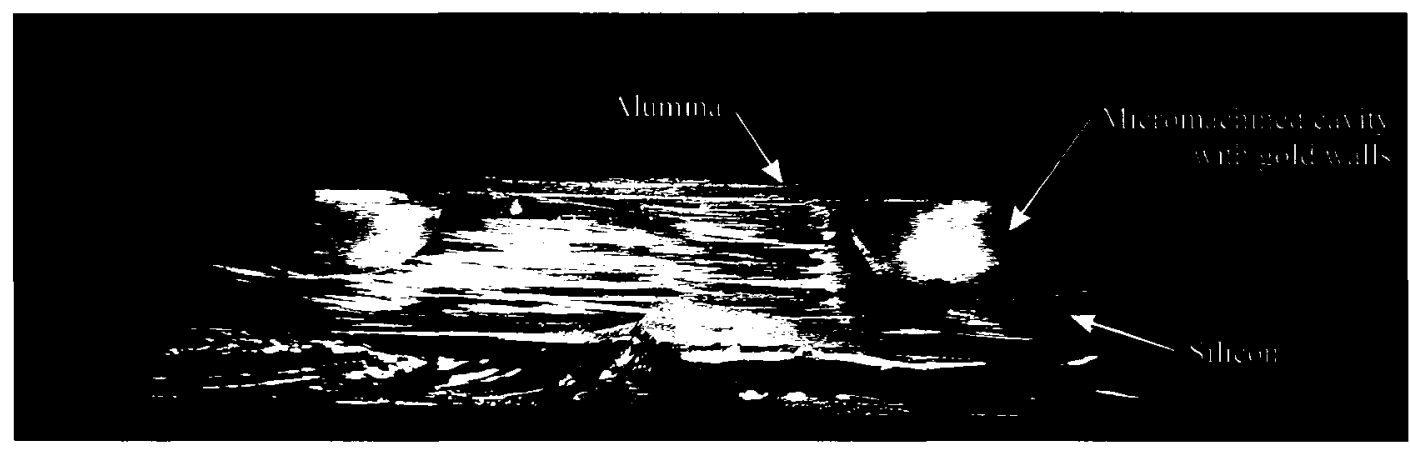

Figure 4.27: Cross-section of a micromachined cavity soldered to an alumina feed wafer using indium.

\subsection{Final Prototype Fabrication}

The design described in Chapter 3, and the fabrication steps developed in Sections 4.1 to 4.4 , were used to produce four prototype micromachined cavities for testing. These prototype cavities are shown in Figures 4.28 (after etching, but before soldering) and 4.29 (after the assembly was completed).

The cavities were micromachined in a quarter-silicon wafer using self-limited isotropic etching and then sputtered with gold. Next they were aligned, using a flip-chip bonder, to an alumina wafer that was previously patterned with microstrip feed lines each having a $0.18 \mathrm{~mm}$ radius aperture in the ground plane for coupling to each cavity (the ground plane forms the top of the cavities). After alignment, the silicon and the alumina were soldered together using a thin indium layer that was previously patterned on the back of the alumina. The full micromachining procedure is included as Appendix A.

During the assembly the alumina wafer broke leaving the four cavities for testing that are indicated in Figures 4.28 and 4.29. The remaining uncovered cavities were not assembled with the remaining piece of alumina so that the inside of the other resonators could be inspected after testing.

Finally, there is an unintentionally etched area that slightly deformed a portion of 


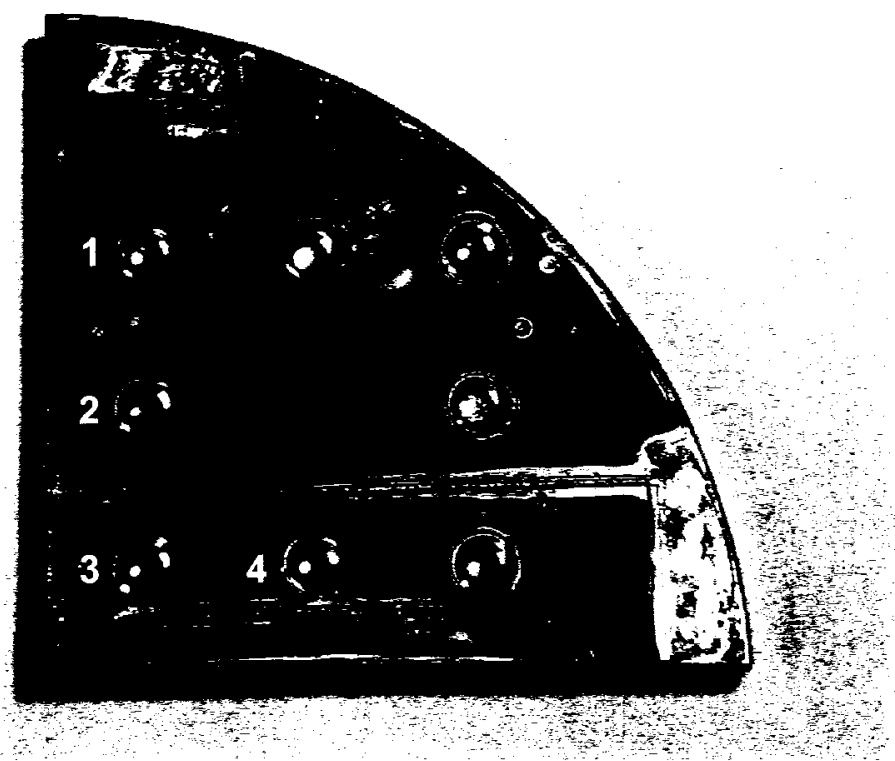

Figure 4.28: Prototype micromachined cavities for testing (after etching but before soldering).

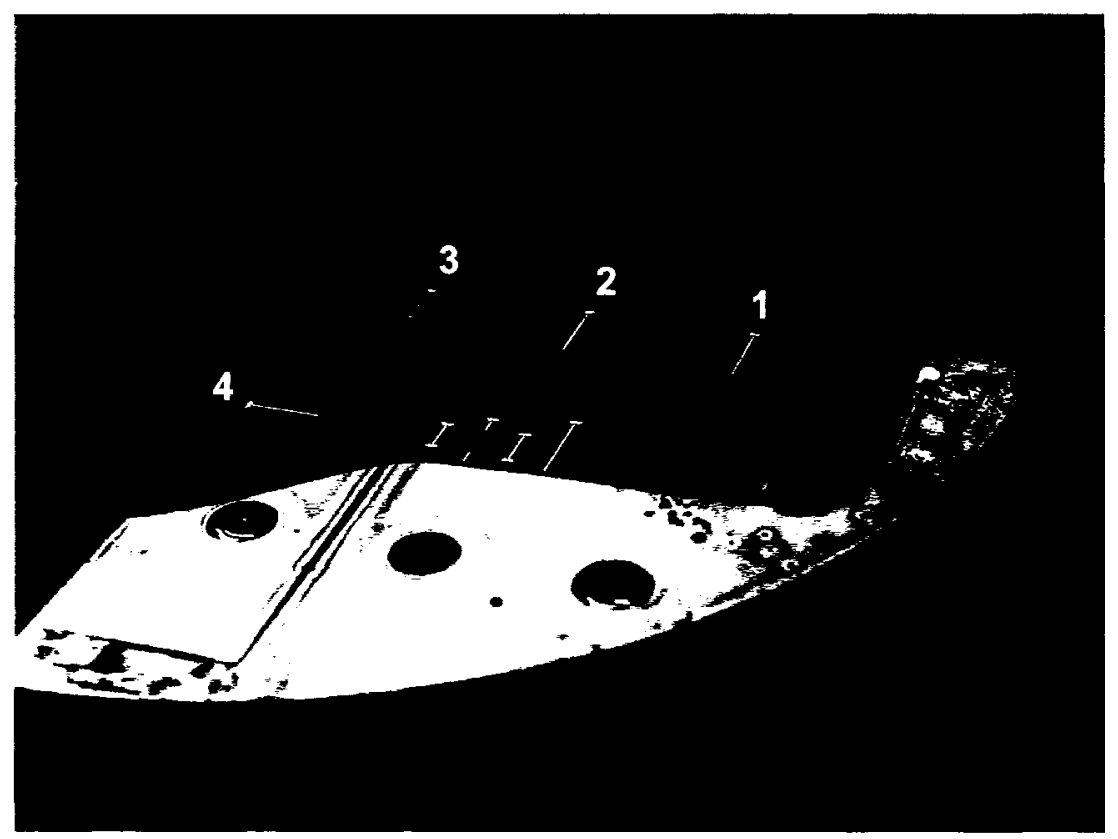

Figure 4.29: Fully-assembled prototype micromachined cavities ready for testing. 
the edges of cavities 3 and 4 . Seen touching the lower edges of cavities 3 and 4 in Figure 4.28, this unintentionally etched horizontal line was caused by an accidental crack in the etchant mask (the polyethylene tape) during the HNA etching. The deformity results in higher roughness (thus more loss) and accordingly cavities 3 and 4 have slightly degraded performance compared with cavities 1 and 2 (measurements in Chapter 5). 


\section{Chapter 5}

\section{Performance of Micromachined}

\section{Hemispherical Cavity Resonators}

The first section of this chapter presents the measured performance of the prototype micromachined hemispherical cavities shown in Figures 4.28 and 4.29. Notably, cavity 1 has a single measured resonance at $76.39 \mathrm{GHz}$ with an unloaded quality factor of 1426 and a loaded quality factor of $\mathbf{9 0 9}$. The second section of this chapter compares this micromachined cavity performance to measurements of conventional hemispherical cavities machined in brass. The final section predicts the possible phase noise improvement of an oscillator using a prototype micromachined hemispherical cavity.

\subsection{Micromachined Cavity Measurements}

Measurements of the four prototype micromachined cavities described in Section 4.5 (Figures 4.28 and 4.29) were performed up to $85 \mathrm{GHz}$ using an Agilent 8510XF network analyzer and $1 \mathrm{~mm}$ ground-signal-ground wafer probes. Calibration structures in the centre of the alumina wafer allowed the probe pads to be de-embedded using TRL calibration. 
The S-parameters of each cavity resonator were measured and then QZERO [163] was used to calculate the measured quality factors. This is the same method to find quality factors for the simulations in Section 3.5.

The measured S-parameters for the four prototype cavities are shown in Figures 5.1 to 5.4. The measured resonant frequencies, unloaded and loaded quality factors are:

- Cavity 1: $f_{o}=76.39 \mathrm{GHz}, Q_{o}=1426, Q_{l}=909$

- Cavity 2: $f_{o}=73.43 \mathrm{GHz}, Q_{o}=1453, Q_{l}=915$

- Cavity 3: $f_{o}=71.51 \mathrm{GHz}, Q_{o}=1241, Q_{l}=833$

- Cavity 4: $f_{o}=75.81 \mathrm{GHz}, Q_{o}=1352, Q_{l}=886$

All four measured resonant frequencies fall within the expected range of $70-83 \mathrm{GHz}$ from the $10 \%$ HNA etching variation described in Section 3.1. Also as expected, cavities 1 and 2 have slightly higher quality factors than cavities 3 and 4 due to the unintentional etching of cavities 3 and 4 previously described in Section 4.5. All four cavities are undercoupled since, as shown in Figure 5.5, each measured $S_{11}$ does not encircle the centre of the Smith Chart.

The sizes of the four prototype cavities were measured prior to assembly. These diameters and depths can be used to calculate and simulate each resonant frequency which can then be compared to the measured value for each prototype cavity. The depths were measured using a DekTak Profilometer while the diameters were measured using micrometers attached to an optical microscope. A typical prototype cavity upper edge is fairly circular but not exactly so, hence a diameter measurement has a small amount of error associated with it. Furthermore, the upper edge of the prototype cavities have a slight overhang (see Figure 4.10) so the diameter seen when using the microscope is 


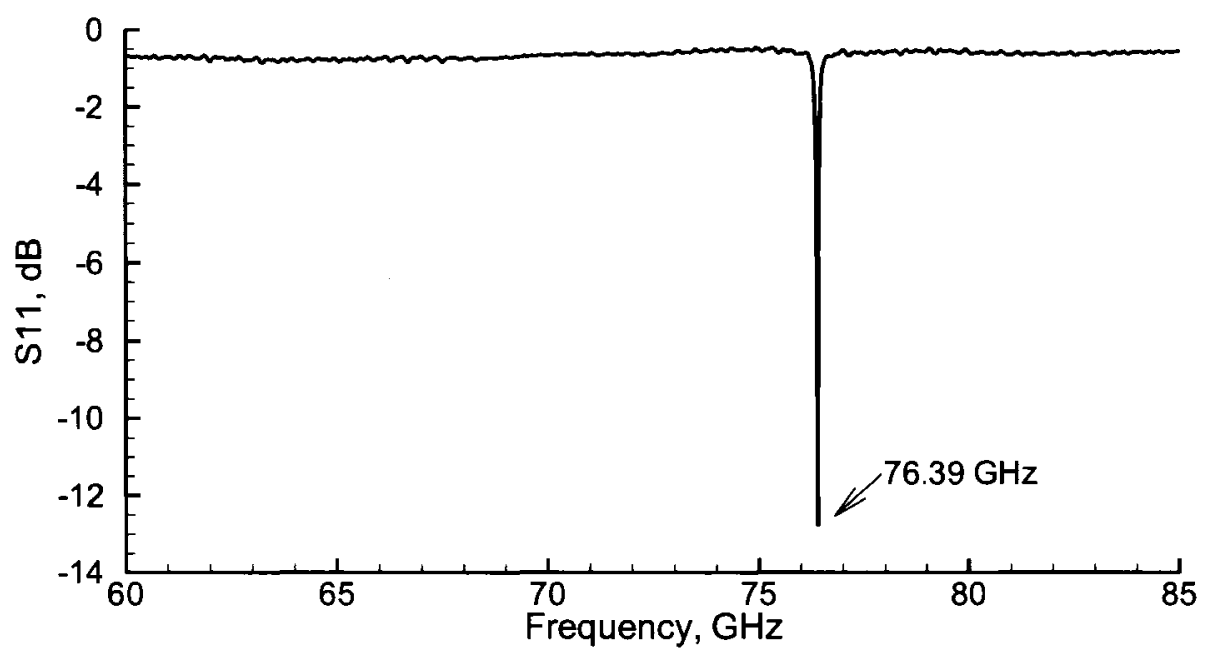

Figure 5.1: Measured $S_{11}$ for micromachined prototype cavity 1 .

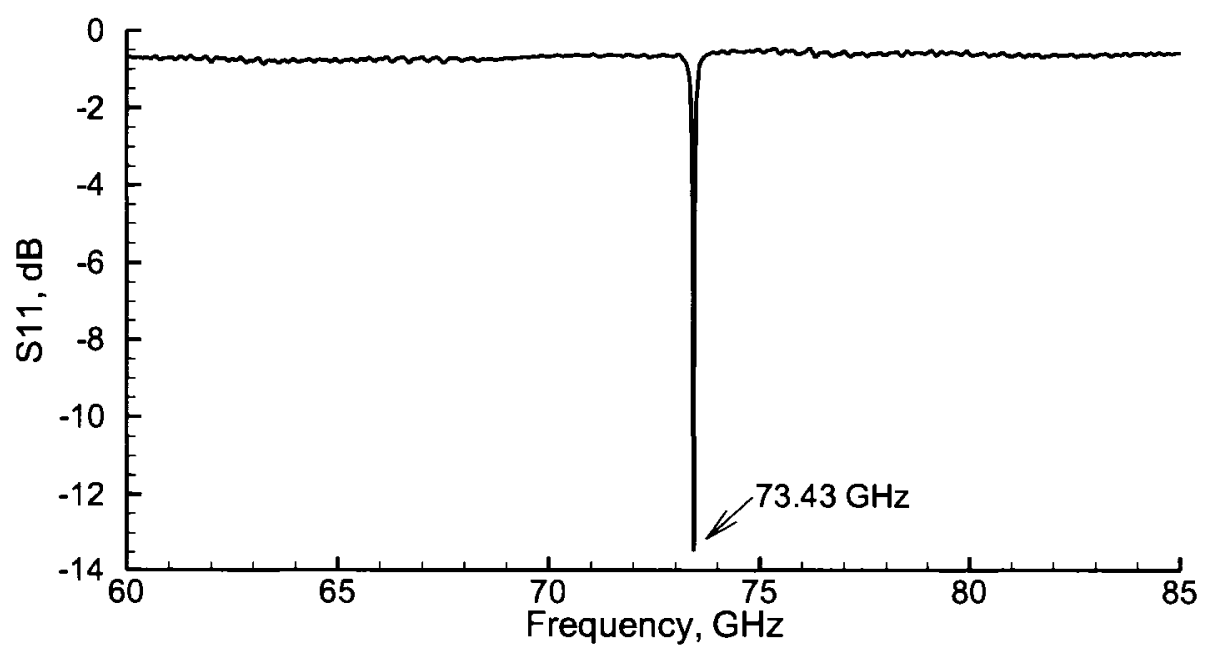

Figure 5.2: Measured $S_{11}$ for micromachined prototype cavity 2 . 


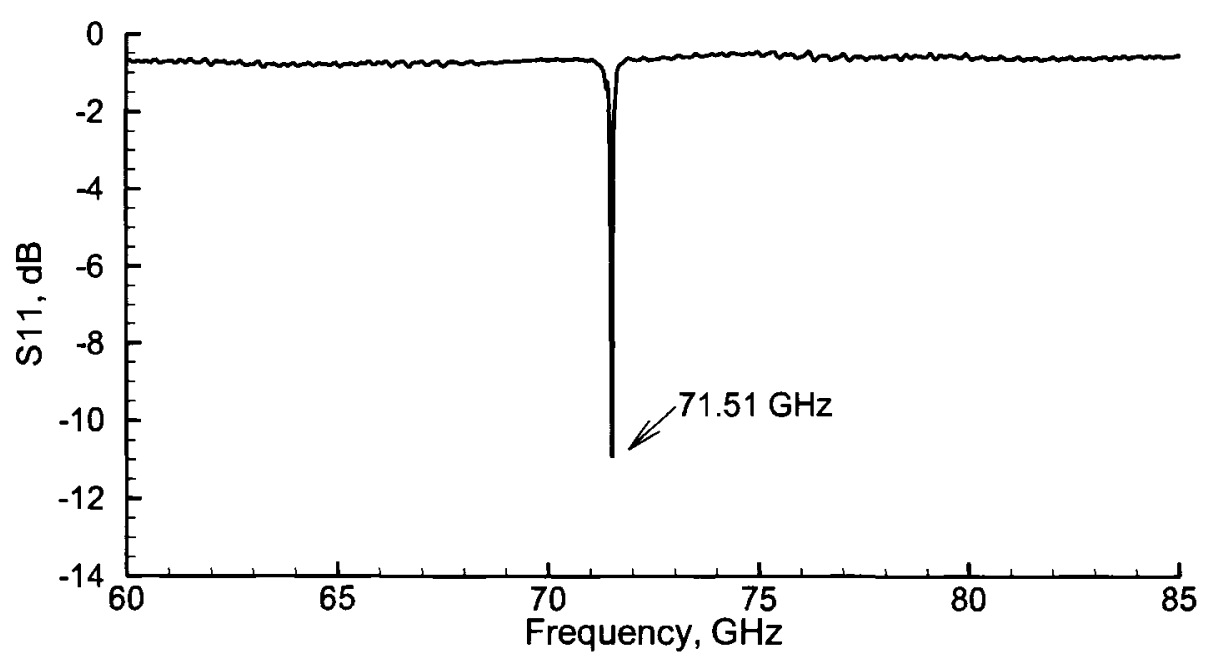

Figure 5.3: Measured $S_{11}$ for micromachined prototype cavity 3 .

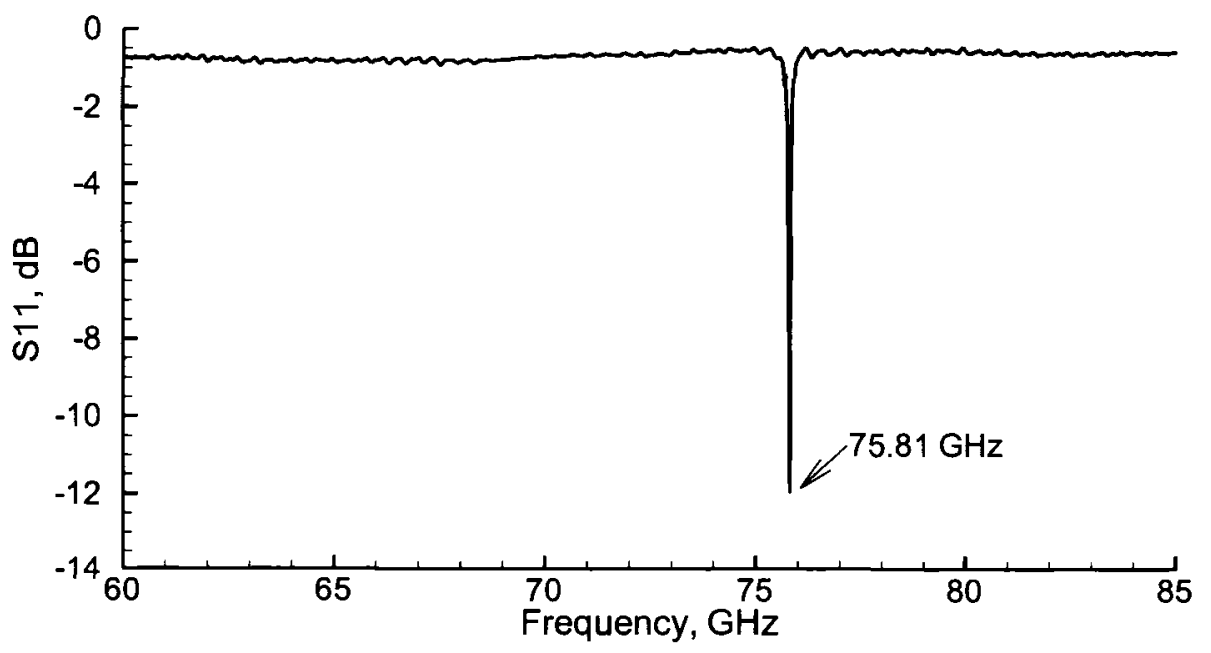

Figure 5.4: Measured $S_{11}$ for micromachined prototype cavity 4 . 
Cavity 1

Span: 76.2-76.5 GHz

Marker: $76.39 \mathrm{GHz}$

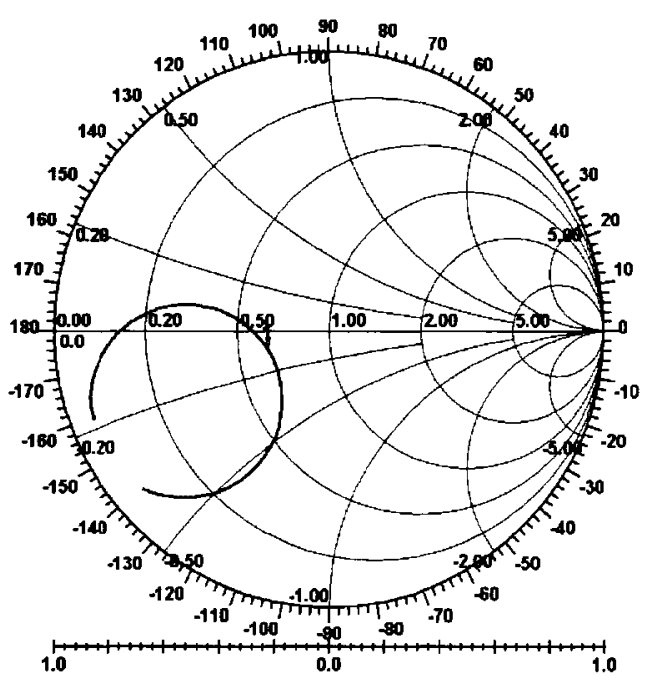

Cavity 3

Span: $71.4-71.6 \mathrm{GHz}$

Marker: $71.51 \mathrm{GHz}$

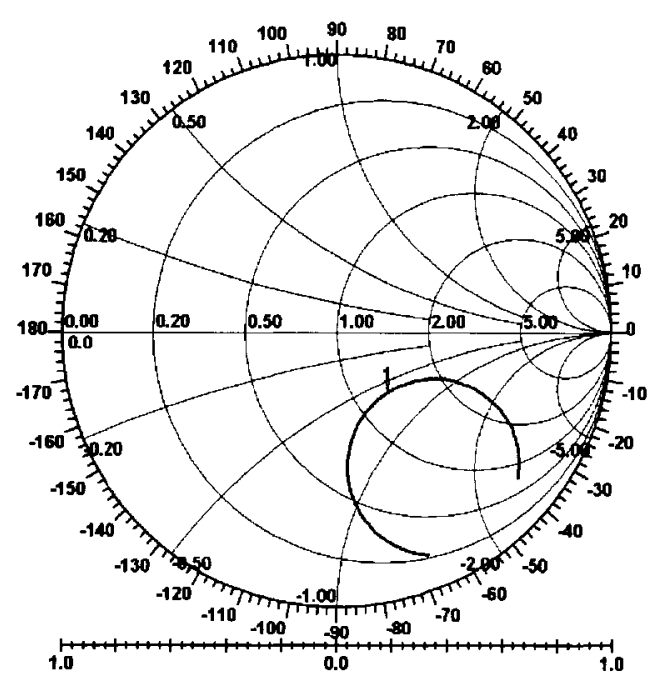

Cavity 2

Span: $73.3-73.5 \mathrm{GHz}$

Marker: $73.43 \mathrm{GHz}$

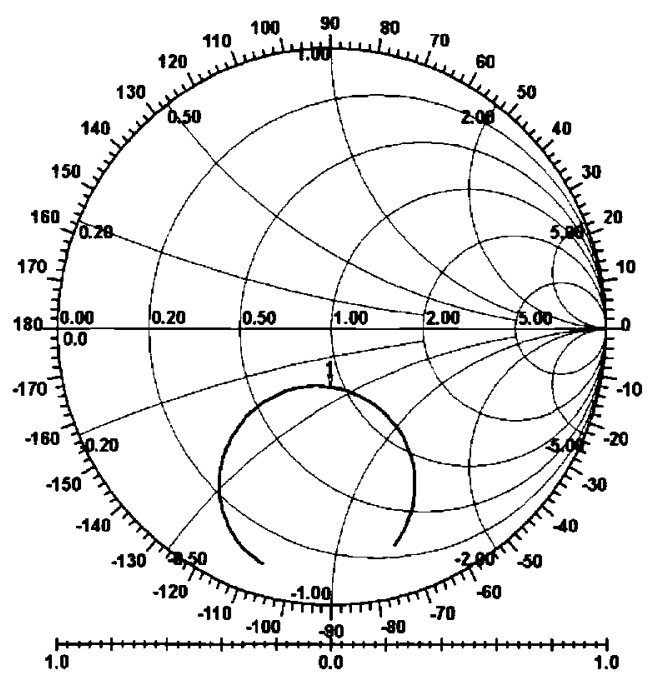

Cavity 4

Span: $75.7-75.9 \mathrm{GHz}$

Marker: $75.81 \mathrm{GHz}$

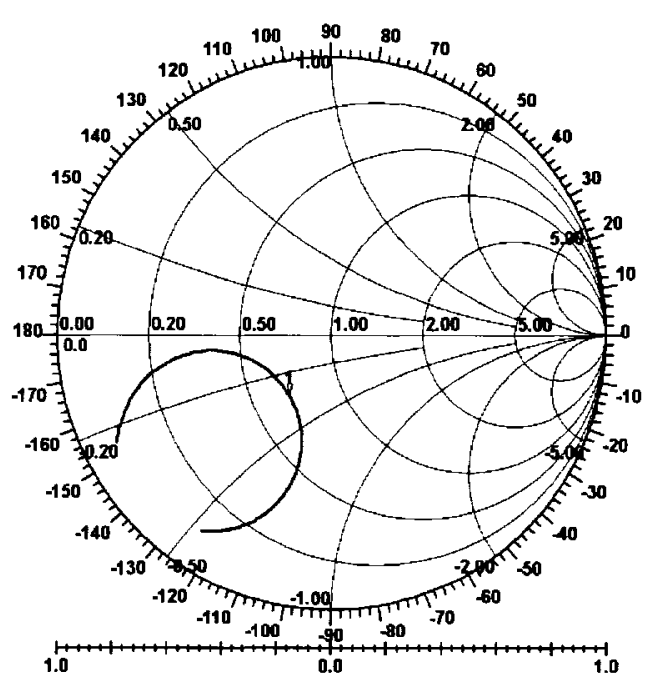

Figure 5.5: Measured $S_{11}$ for micromachined prototype cavities 1 to 4 . 


\begin{tabular}{|c||c|c||c|c|c|}
\hline \multicolumn{1}{|c||}{ Cavity } & \multicolumn{2}{c||}{ Measured Size, mm } & \multicolumn{4}{c|}{ Resonant Frequency, GHz } \\
& Radius & Depth & Calculated & Simulated & Measured \\
\hline \hline 1 & 1.705 to 1.780 & 1.200 & 74.28 to 77.44 & 74.19 to 77.43 & 76.39 \\
2 & 1.762 to 1.837 & 1.200 & 72.08 to 75.03 & 72.01 to 74.91 & 73.43 \\
3 & 1.789 to 1.864 & 1.235 & 70.97 to 73.84 & 70.92 to 73.80 & 71.51 \\
4 & 1.711 to 1.786 & 1.203 & 74.05 to 77.19 & 73.98 to 77.11 & 75.81 \\
\hline
\end{tabular}

Table 5.1: Calculated, simulated and measured resonant frequencies versus the range of possible physical size for each of the prototype micromachined cavities.

slightly smaller than the true diameter due to this inward perturbation. These two factors make exact measurements of the cavity diameters difficult and it is estimated that $75-150 \mu \mathrm{m}$ must be added to the radius measured using the microscope. Table 5.1 lists the measured sizes of the four prototype cavities and the corresponding range of calculated (equation 3.2) and simulated (Section 3.3) resonant frequencies. The measured values, also listed in Table 5.1, fall within both the calculated and simulated resonant frequency ranges. Alternatively, the measured resonant frequencies can be used to determine the actual cavity sizes by using equation 3.2 for resonant frequency and solving for cavity radius. This results in actual cavity radii of $1.731 \mathrm{~mm}, 1.805 \mathrm{~mm}, 1.854 \mathrm{~mm}$ and $1.745 \mathrm{~mm}$, respectively, which are all within the measured size ranges listed in Table 5.1.

In addition to cavity size, the actual cavity surface resistance is needed to compare both the calculated and simulated unloaded quality factors to the measured unloaded quality factor. The difficulty is, as seen in Section 3.2, that the actual surface resistance can be anywhere between $\mathcal{R}$ to $2 \mathcal{R}$. Equation 2.10 defines the ideal surface resistance, $\mathcal{R}$, which assumes dc conductivity, perfect shape, perfect solder bond, perfect alignment and no roughness. All of these assumptions are difficult, if not impossible, to accurately quantify, thus making the determination of the individual loss contributions problematic. Therefore, to determine the actual surface resistance, equation 3.10 for the unloaded $\mathrm{Q}$ was equated to the measured value and then solved for the actual surface resistance as 


\begin{tabular}{|c||c|c|c||c|c|c|}
\hline \multicolumn{1}{|c||}{ Cavity } & \multicolumn{3}{c||}{ Unloaded Quality Factor } & \multicolumn{3}{c|}{ Loaded Quality Factor } \\
& Calculated & Simulated & Measured & Calculated & Simulated & Measured \\
\hline \hline 1 & 1423 & 1399 & 1426 & 909 & 933 & 909 \\
2 & 1453 & 1347 & 1453 & 965 & 906 & 915 \\
3 & 1246 & 1157 & 1241 & 892 & 836 & 833 \\
4 & 1358 & 1324 & 1352 & 890 & 891 & 886 \\
\hline
\end{tabular}

Table 5.2: Calculated, simulated and measured quality factors for each of the prototype micromachined cavities.

a multiple of the ideal surface resistance [173]. For prototype cavities 1-4 the actual surface resistance is $1.49 \mathcal{R}, 1.54 \mathcal{R}, 1.86 \mathcal{R}$ and $1.58 \mathcal{R}$, respectively. For cavities 1 and 2 the measured loss is about 1.5 times higher than the ideal loss and, as expected, cavities 3 and 4 have more loss due to the unintentionally etched edge. Although factors like the actual shape, any slight misalignment and the actual quality of the solder bond all contribute to the actual loss it is worth noting that since the skin depth at $77 \mathrm{GHz}$ is only about $0.283 \mu \mathrm{m}$ it takes very little roughness to cause loss. The roughness of the alumina and the bottom of the cavities appear to be smaller than the skin depth, however, the upper sides of the cavities are visually rougher than the bottoms and likely contribute to the loss. The indium is rough also, but it is thin so although its roughness contributes it likely doesn't dominate the cavity loss.

With the actual cavity size and loss known, the calculated and simulated quality factors can be accurately compared to the measured quality factors. This is shown in Table 5.2. The correlation between the calculated, simulated and measured loaded quality factors is good for all four prototype cavities. Since the calculated, simulated and measured loaded quality factors agree then the equations from Chapter 3 may be used to predict the performance of other aperture sizes rather than having to resort to more lengthy simulations.

Finally, simulating the actual size and cavity loss results in simulated response that 


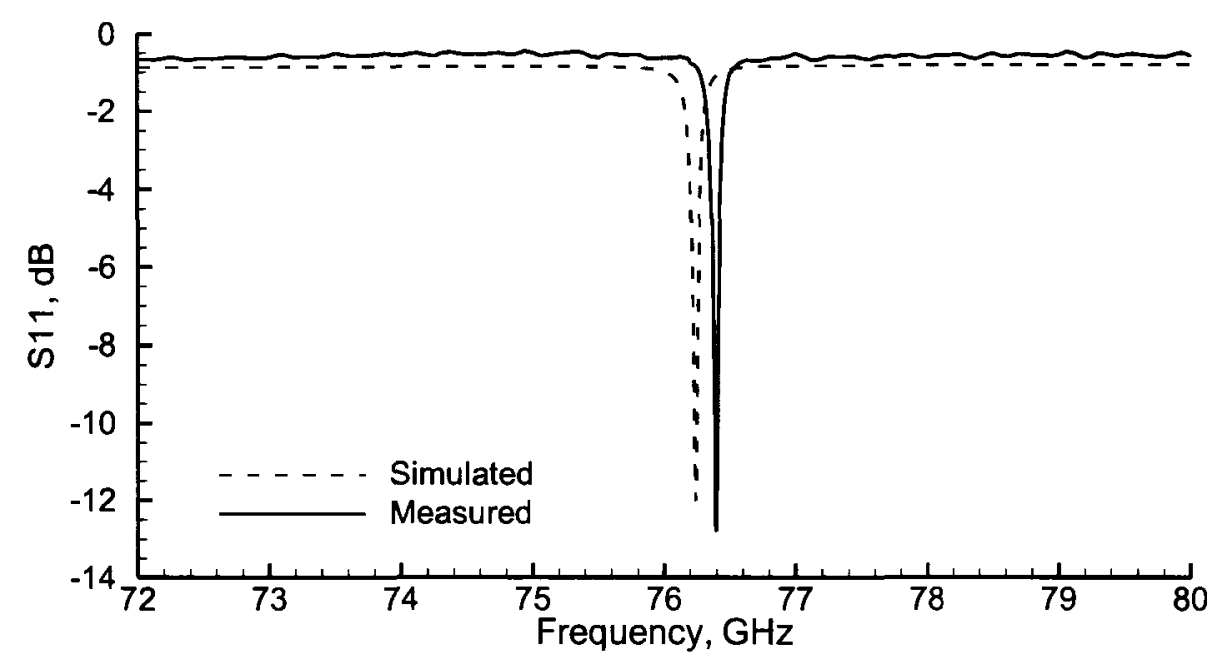

Figure 5.6: Simulated $S_{11}$ using the actual size and loss for prototype micromachined cavity 1 along with its corresponding measured $S_{11}$.

closely predicts the measured response, as is shown in Figure 5.6 (for cavity 1).

\subsection{Comparison to Machined Cavities}

During the fabrication of the micromachined prototypes, other cavities with the same geometry were manufactured in parallel using conventional machining techniques. The original purpose of these machined cavities was to help troubleshoot issues encountered during the development of the micromachined cavities by having somewhat similar samples that were produced by then more-controllable conventional machining techniques. The completed machined cavities provide another benefit, however, of being a direct comparison to the micromachined prototype cavities since the same design outlined in Chapter 3 was used for both.

The machined cavities were made using brass and are shown in Figures 5.7 (after machining but before soldering) and 5.8 (after the assembly was completed). The brass 


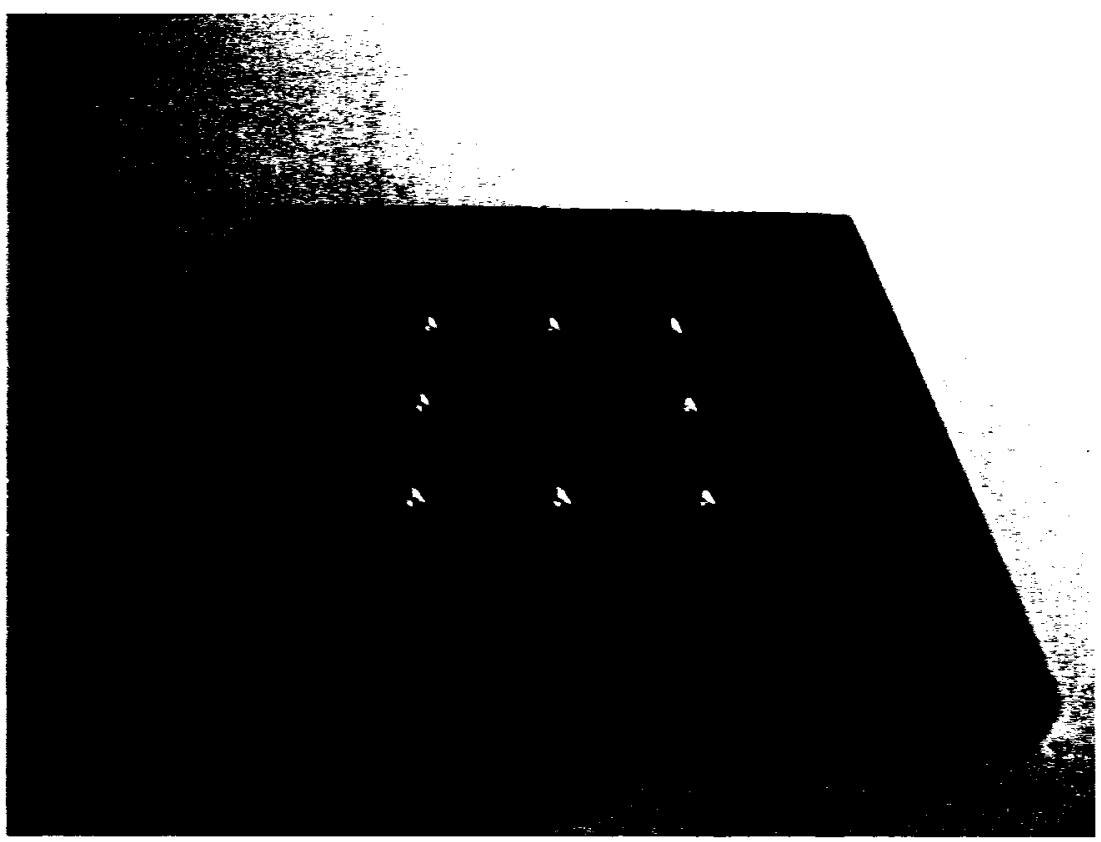

Figure 5.7: Machined brass cavities ready for assembly.

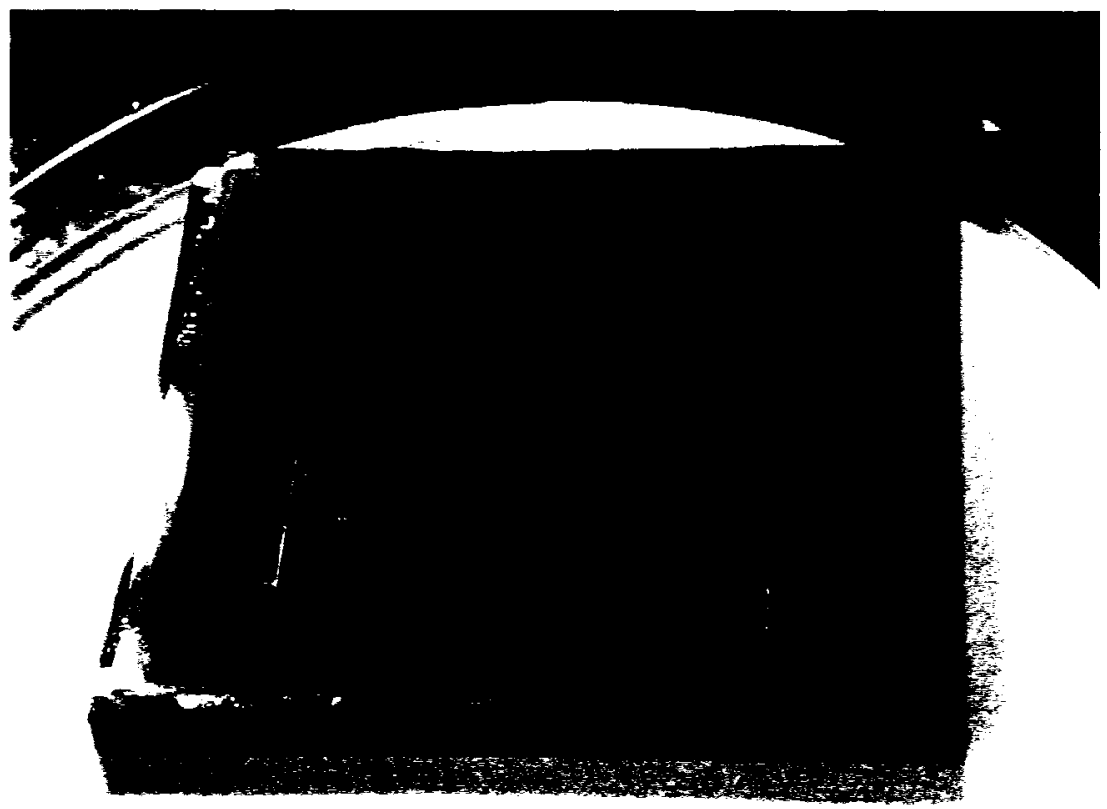

Figure 5.8: Assembled brass cavities. 


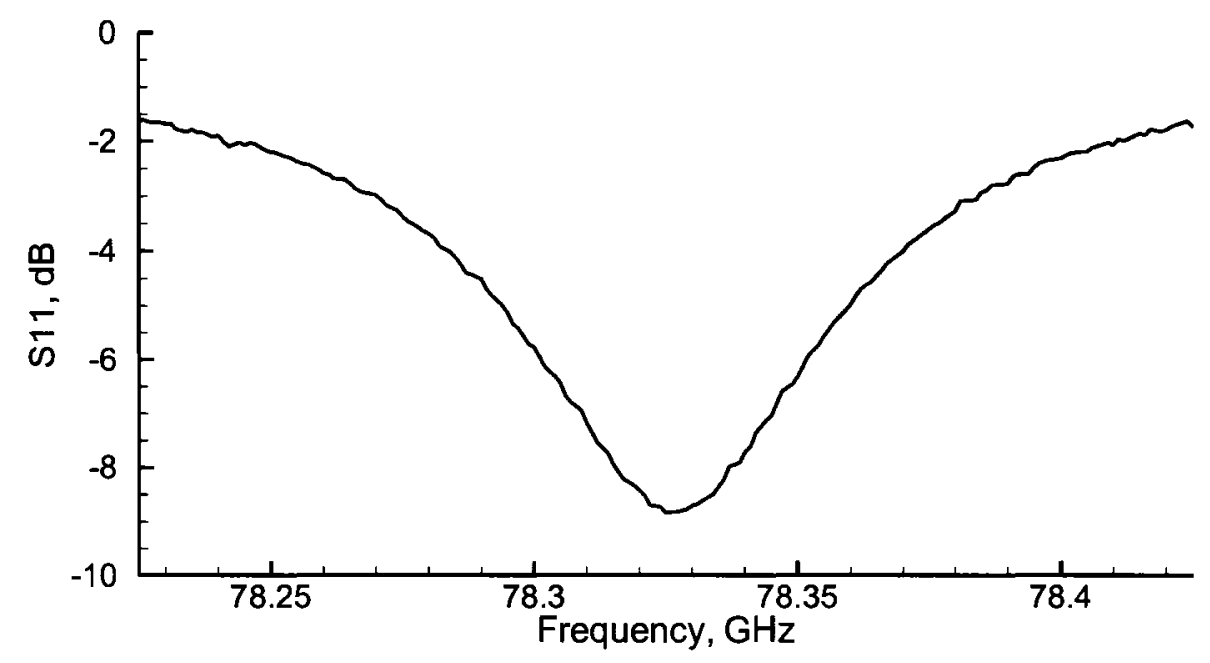

Figure 5.9: Measured $\mathrm{S}_{11}$ of an oblate hemispherical cavity fabricated in brass using traditional machining.

cavities were slightly smaller than the micromachined cavities (due to the machining tolerance), however, in all other geometric aspects are the same. An alumina wafer with the same back/front patterning was aligned and soldered to the brass using a similar indium soldering procedure. A typical brass cavity had a measured resonant frequency of $78.33 \mathrm{GHz}$, as shown in Figure 5.9, an unloaded quality factor of 1016 and a loaded quality factor of 728 . The quality factors of the conventional brass cavities are lower than the prototype micromachined cavities, and this difference is primarily due to a surface roughness of $3 \mu \mathrm{m}$, which is much rougher than the micromachined cavities.

\subsection{Phase Noise Improvement}

The previous sections show that high loaded quality factors can be achieved using the prototype micromachined cavity resonators, however, the question that remains is how much does this improvement lower the phase noise of an oscillator using a prototype 
micromachined cavity? This section will show that the phase noise may be decreased by approximately -20 to $-30 \mathrm{dBc} / \mathrm{Hz}$ at $\mathrm{W}$-band when compared to a $\mathrm{W}$-band oscillator using a conventional distributed resonator.

The figure of merit for oscillator phase noise is the normalized single sideband noise spectral density, $\mathcal{L}$, which is commonly approximated by Leeson's phase noise model [1]:

$$
\mathcal{L}(\Delta \omega)=10 \log \left\{\frac{2 F k T}{P_{\text {sig }}}\left[1+\left(\frac{\omega_{o}}{2 Q_{l} \Delta \omega}\right)^{2}\right]\left(1+\frac{\Delta \omega_{1 / f^{3}}}{|\Delta \omega|}\right)\right\}
$$

where $F, k, T, P_{s i g}, \Delta \omega_{1 / f^{3}}$ are parameters of the oscillator, $Q_{l}$ is the loaded quality factor, $\omega_{0}$ is the carrier frequency and $\Delta \omega$ is the offset from the carrier where $\mathcal{L}$ is being characterized. Leeson's equation can be used to predict how much the phase noise can be lowered by using a prototype micromachined cavity compared to using an existing W-band resonator.

Consider first the phase noise, at the same carrier offset, of two $77 \mathrm{GHz}$ oscillators identical except that each has a different resonator with a different loaded quality factor, $Q_{l, h i g h}$ and $Q_{l, l o w}$. In this case, the parameters $F, k, T, P_{s i g}, \Delta \omega_{1 / f^{3}}, \omega_{o}$ and $\Delta \omega$ are approximately the same for both and then equation 5.1 for each can be approximated by

$$
\mathcal{L}_{1}=10 \log \left(\frac{\mathcal{K}}{Q_{l, \text { low }}^{2}}\right) \text { and } L_{2}=10 \log \left(\frac{\mathcal{K}}{Q_{l, \text { high }}^{2}}\right)
$$

where $\mathcal{K}$ is a constant. If $Q_{l, l o w}<Q_{l, h i g h}$ then the amount the phase noise is decreased by using the higher quality factor resonator $\left(Q_{l, h i g h}\right)$ compared to the lower quality factor resonator $\left(Q_{l, l o w}\right)$ is

$$
\Delta \mathcal{L}=\mathcal{L}_{2}-\mathcal{L}_{1}=10 \log \left(\frac{\mathcal{K}}{Q_{l, h i g h}^{2}}\right)-10 \log \left(\frac{\mathcal{K}}{Q_{l, \text { low }}^{2}}\right)=10 \log \left(\frac{Q_{l, \text { low }}^{2}}{Q_{l, \text { high }}^{2}}\right)
$$

A plot of equation 5.3 as a function of the loaded quality factor ratio shows the decrease in 


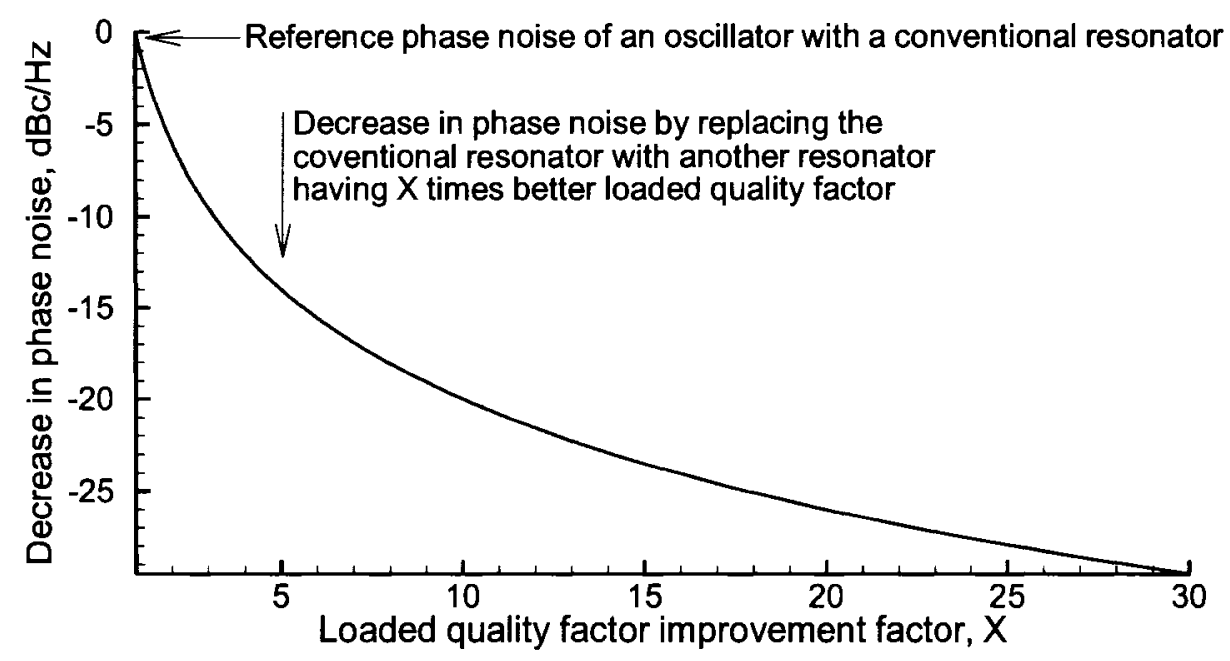

Figure 5.10: Phase noise decrease when a conventional resonator is replaced with a resonator having $\mathrm{X}$ times higher loaded quality factor, such as a prototype micromachined cavity.

phase noise as a function of the loaded quality factor improvement that a micromachined cavity $\left(Q_{l, h i g h}\right)$ has over a conventional resonator $\left(Q_{l, l o w}\right)$. This is given in Figure 5.10 and it shows that when the micromachined cavity has a loaded quality factor of up to 10 times that of a conventional resonator there is a rapid decrease in phase noise (up to -20 $\mathrm{dBc}$ ). If the loaded quality factor improvement is more than 10 times then there is still a decrease in phase noise, however, it is not as pronounced.

Equation 5.3 and Figure 5.10 can be used to estimate the decrease in phase noise if a W-band prototype micromachined cavity were used in place of the W-band, multiplering, thin-film microstrip resonator [10] previously mentioned in the Introduction. This microstrip resonator is a good example of state-of-the-art performance in a standard IC process where a loaded quality factor of $Q_{l}=38$ was reported in $0.18 \mu \mathrm{m}$ CMOS. Using the measured loaded quality factor of $Q_{l}=909$ for the prototype micromachined cavity, then equation 5.3 estimates that the micromachined cavity would lower the phase noise 
by $-27.58 \mathrm{dBc}$ at $77 \mathrm{GHz}$.

This is a large improvement and is not unexpected since the losses in the silicon thinfilm microstrip resonator are significant. The prototype micromachined cavity is therefore also compared to a conventional microstrip half-wave resonator on a lower loss substrate such the 5 mil Alumina Superstrate TPS used to feed the cavities (alumina has $\epsilon_{r}=9.9$, $\tan \delta=0.0001)$. The microstrip line is assumed to have a characteristic impedance of $50 \Omega$ and is thus $129 \mu \mathrm{m}$ wide, has an effective dielectric constant of $\epsilon_{e f f}=7.201$ and a surface resistance of $\mathcal{R}_{s}=\sqrt{\frac{\omega \mu_{o}}{2 \sigma}}\left(\sigma=4.098 \times 10^{7} \mathrm{~S} / \mathrm{m}\right.$ for gold $)$. This microstrip half-wave resonator would have an unloaded quality factor at $77 \mathrm{GHz}$ of $Q=\frac{\beta}{2 \alpha}=162$ where the propagation constant is $\beta=\frac{\omega \sqrt{\epsilon_{e f f}}}{3 \times 10^{8}}$ and the total attenuation, $\alpha$, is the sum of the conductor loss $\left(\frac{R_{s}}{Z_{o} W}\right)$ and the dielectric loss $\left(\frac{k_{o} \epsilon_{r}\left(\epsilon_{e f f}-1\right) \tan \delta}{2 \sqrt{\epsilon_{e f f}}\left(\epsilon_{r}-1\right)}\right)$ [11]. Finally, assuming that loaded quality factor is about two-thirds of the unloaded quality factor (since this condition has been shown to result in the lowest phase noise for some oscillators [18]) the loaded quality factor for the half-wave microstrip on alumina is about 100 . Comparing this to the measured loaded quality factor of 909 for the prototype micromachined cavities results in a estimated decrease in phase noise of $-19.17 \mathrm{dBc}$ if the micromachined cavity was used in place of the half-wave microstrip resonator on alumina. 


\section{Chapter 6}

\section{Conclusion}

This chapter summaries the motivation and results of this research, lists its contributions and outlines possibilities for future work.

\subsection{Summary}

The purpose of this research was to produce a resonator with an improved loaded quality factor at $\mathrm{W}$-band in order to lower the phase noise of W-band oscillators. In Section 1.1 MEMS were presented as a potential technology to achieve high quality factors at W-band, with the most likely candidate being bulk micromachined cavity resonators. Table 2.1 listed the published performance of existing micromachined cavities.

Novel isotropically etched hemispherical cavities were proposed to achieve a higher unloaded quality factor than that of existing micromachined cavities. Additionally, optimized coupling was proposed to maximize the loaded quality factor as well. Therefore, the goal was to produce a micromachined hemispherical cavity that resonates at $\mathrm{W}$-band with an unloaded quality factor greater than 1000 and a loaded quality factor greater than 500 . 
A custom micromachining procedure was developed based on self-limited etching and a new indium soldering assembly process. Novel vialess probing was also explored to eliminate the need for drilled substrate vias. The design was accomplished using a newly developed theoretical analysis based on equivalent energy aperture coupling theory and perturbation techniques. The resulting theoretical design was compared to $3 \mathrm{D}$ finite element simulations of the resonator and although the analytical basis for these two approaches are quite different they were found to be in good agreement.

The development steps for a target resonant frequency of $77 \mathrm{GHz}$ are summarized as follows:

1. Calculate the micromachined cavity radius using equation 3.1

$\rightarrow$ Result: $1.704 \mathrm{~mm}$

2. Calculate the nominal resonant frequency using equation 3.2

$\rightarrow$ Result: $77.670 \mathrm{GHz}$

3. Calculate the unloaded quality factor, with realistic loss, using equation 3.10

$\rightarrow$ Result: 1381

4. Calculate the loaded quality factor using equation 3.26 (with equations $3.10,3.12$, $3.14,3.20,3.25)$

$\rightarrow$ Result: Figure 3.8. For aperture radii of $0.12 \mathrm{~mm}, 0.15 \mathrm{~mm}$ and $0.18 \mathrm{~mm}$ the calculated loaded quality factors are 1298,1125 and 875 , respectively

5. Simulate the unloaded resonant frequency and unloaded quality factor, with realistic loss

$\rightarrow$ Result: $77.682 \mathrm{GHz}$ and 1392, respectively. Other modes in Table 3.1. Fields in Figure 3.5 
6. Simulate the loaded resonant frequency and loaded quality factor

$\rightarrow$ Result: Figure 3.14. For aperture radii of $0.12 \mathrm{~mm}, 0.15 \mathrm{~mm}$ and $0.18 \mathrm{~mm}$ the results are $77.61 \mathrm{GHz}, 77.63 \mathrm{GHz}, 77.61 \mathrm{GHz}$ and 1360, 1161, 939, respectively. S-parameters and fields in Figures 3.10 and 3.13

7. Calculate the self-limited etch mask diameter using equation 4.1

$\rightarrow$ Result: $1.667 \mathrm{~mm}$

8. Experiment with HNA etching and determine etchant composition

$\rightarrow$ Result: $2: 3: 3$ of $49 \% \mathrm{HF}, 70 \% \mathrm{HNO}_{3}, 99 \% \mathrm{CH}_{3} \mathrm{C} 00 \mathrm{H}, 70^{\circ} \mathrm{C}$, no agitation

9. Design the feed substrate including $\mathrm{V} / \mathrm{W}$-band vialess probe pads

$\rightarrow$ Result: Microstrip dimensions in Section 4.2. Vialess probe pads in Section 4.3 with measured performance in Figures 4.20 and 4.21

10. Develop plated-indium soldering process using a flip-chip bonder to align and bond the alumina feed to the micromachined silicon cavities

$\rightarrow$ Result: Experiments and final procedure in Section 4.4. Indium thickness: $30 \mu \mathrm{m}$

11. Built and test prototype cavities

$\rightarrow$ Pictured in Figure 4.29

The measured performance of the prototype micromachined cavities is:

- Cavity 1: $f_{o}=76.39 \mathrm{GHz}, Q_{o}=1426, Q_{l}=909$

- Cavity 2: $f_{o}=73.43 \mathrm{GHz}, Q_{o}=1453, Q_{l}=915$

- Cavity 3: $f_{o}=71.51 \mathrm{GHz}, Q_{o}=1241, Q_{l}=833$

- Cavity 4: $f_{o}=75.81 \mathrm{GHz}, Q_{o}=1352, Q_{l}=886$ 
The measured performance of the prototype cavities is very similar to both the calculated and simulated performance as shown in Tables 5.1 and 5.2 and also in Figure 5.6.

An identical cavity design, except for being fabricated in brass using conventional machining, was also built but did not perform as well as the micromachined prototype cavities due to higher losses from a rougher surface.

If two identical W-band oscillators are compared with one using a prototype micromachined cavity and the other using a conventional alumina microstrip distributed resonator, then the oscillator with the micromachined hemispherical cavity would have almost $20 \mathrm{dBc}$ less phase noise compared to the oscillator with the alumina microstrip resonator. When the micromachined hemispherical cavity is compared to a recently published W-band thin-film microstrip resonator in $0.18 \mu \mathrm{m}$ CMOS the phase noise improvement becomes almost $30 \mathrm{dBc}$ lower.

\subsection{Contributions}

This research has contributed a novel resonator structure with the highest measured performance reported at W-band. It was designed using novel theoretical calculations that have been verified using both 3D FEM simulations and measurements. More specifically:

- The first hemispherical micromachined cavity resonator has been successfully built and tested, verifying a new micromachining and assembly procedure. The micromachining uses self-limited etching to define the cavity size and the assembly uses a thin layer of indium to minimize the effect of the solder layer on cavity performance.

- The highest frequency micromachined resonator at $76.39 \mathrm{GHz}$ with the highest unloaded and loaded quality factors of 1426 and 909, respectively, of any micromachined cavity resonator, as shown in Figures 6.1 and 6.2. 


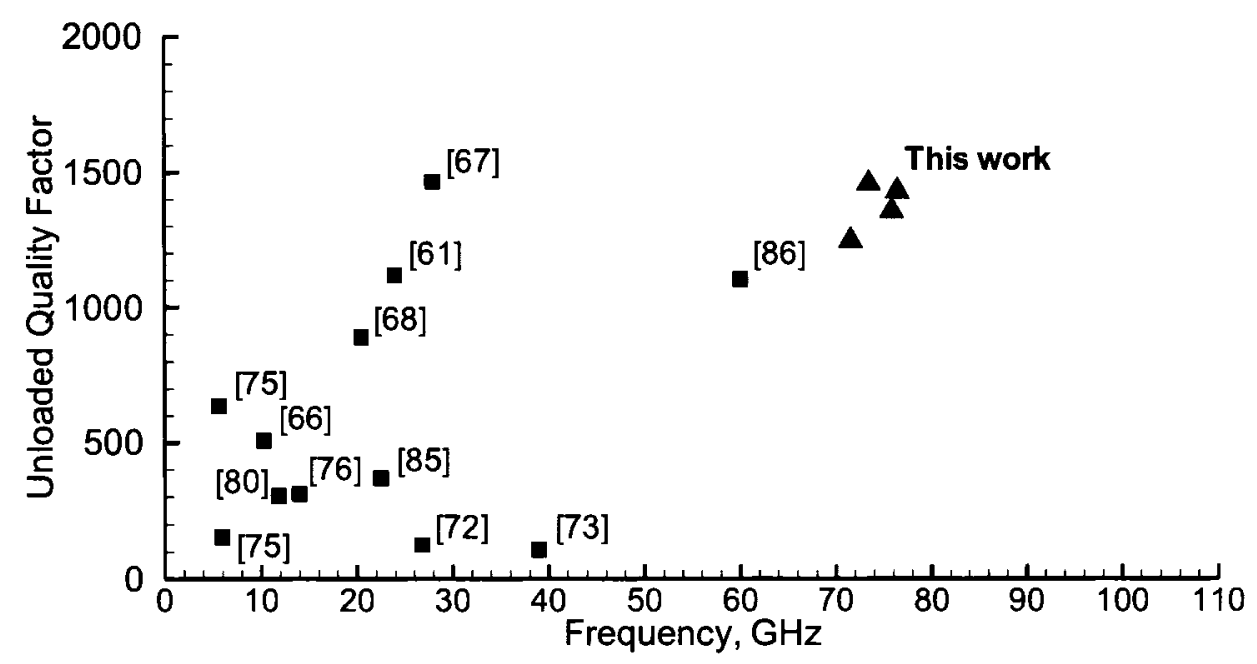

Figure 6.1: Measured unloaded quality factors of the prototype micromachined cavities compared with existing micromachined cavity resonators.

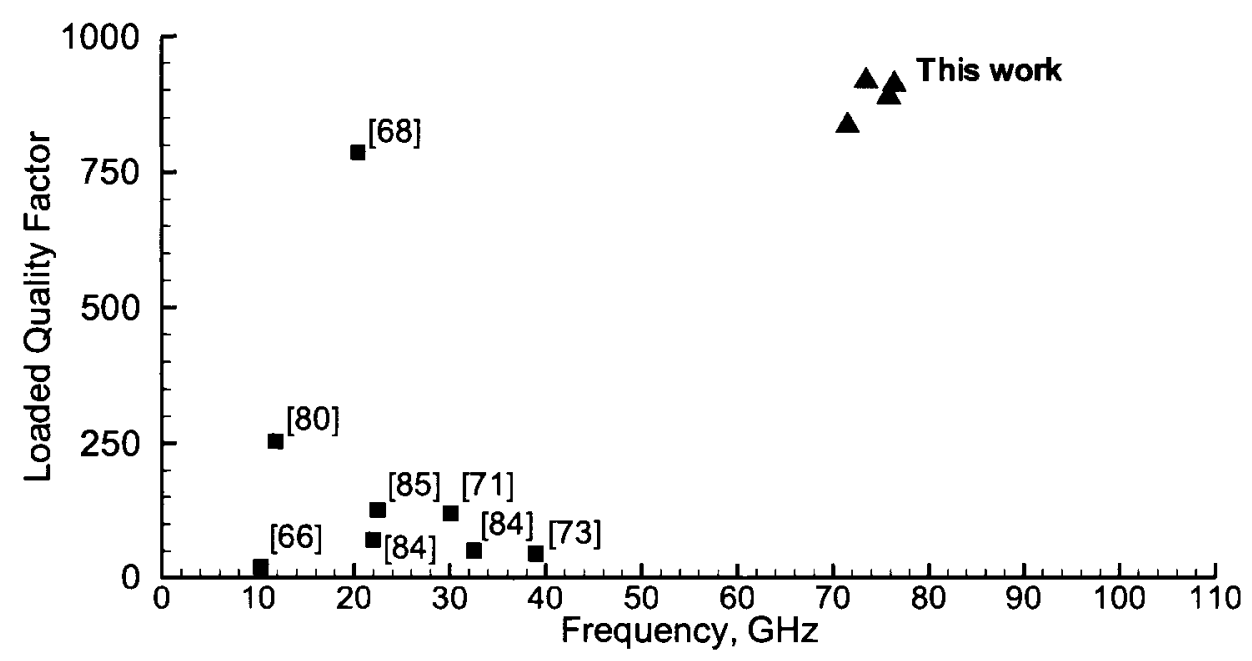

Figure 6.2: Measured loaded quality factors of the prototype micromachined cavities compared with existing micromachined cavity resonators. 
- New design equations have been developed to predict the resonant frequency, the unloaded quality factor and the loaded quality factor of an oblate micromachined hemispherical cavity that is aperture coupled to a microstrip line. The loaded quality factor can be optimized by just varying the aperture size in the calculations.

- The calculated resonant frequency, quality factors and fields agree with both $3 \mathrm{D}$ finite elements simulations of the micromachined cavity as well as measurements of the prototype micromachined hemispherical cavities.

\subsection{Future Work}

Despite the accomplishments and contributions summarized in Sections 6.1 and 6.2, there are issues that remain and may be the subject of future work. One of these issues was the difficulty accurately measuring both the size and roughness of the etched cavities, which in turn limited the accuracy of both the calculations and the simulations. Although more precise measuring equipment may help, knowing the exact shape and roughness profile may not, however, improve the accuracy of the predicted performance. This is because both the calculations and the simulations assume simple values, such as only the radius and depth for size, and only the rms surface roughness expressed as a single value for the entire geometry. Both the size and the roughness are obviously more complicated than these simple values, therefore perhaps the equations and simulations should be improved to use more complicated representations of size and surface roughness. In any event, more accurate techniques to measure the size and roughness of the cavities would help improve the accuracy of the predicted performance.

A more important issue is the size variation from cavity to cavity, which causes the resonant frequency to vary as well. These variations were not unexpected since other published applications of self-limited etching also report similar results. Furthermore, 
this research developed a single custom micromachining procedure, that is, the prototype cavities measured and presented here were produced using the first successful fabrication procedure. No effort was made to further optimize or research this fabrication procedure, and hence no attempt was made to reduce the size variation from cavity to cavity by improving the etching procedure. This first developed micromachining procedure is like all early process development work: capable of producing functioning cavities, however, likely not optimal. This is, therefore, an important area of future research. If the yield of the cavities is defined as being close to a target frequency, then more work is required. On the other hand, if the yield is defined as micromachined cavities that resonate, then the current fabrication procedure can be considered a success. On the wafer tested, $75 \%$ of the cavities were etched successfully. Of the etched cavities aligned and soldered to the feed substrate, $100 \%$ resonated with frequencies and quality factors predicted by both calculations and simulations. Nevertheless, the self-limited etching should be further investigated to find an etching procedure that has less size variation from cavity to cavity.

A suggestion to improve the cavities may be to use them at higher frequencies. A higher resonant frequency requires a smaller cavity which in turn means that both smaller mask openings and shorter etching times can be used. A shorter etching time may result in less size variation from cavity to cavity. A smaller mask opening may result in better sphericity of the etched geometry. Early etching experiments of smaller cavities seemed to point to these conclusions, although with resonant frequencies of at least $130 \mathrm{GHz}$, these could not be pursued due to the $85 \mathrm{GHz}$ upper frequency limitation of the measurement equipment. Nevertheless, etching smaller cavities that are much closer to perfect hemispheres would likely have near-optimum performance and hence likely produce some of the highest quality factors possible for fundamental mode micromachined cavity resonators. 


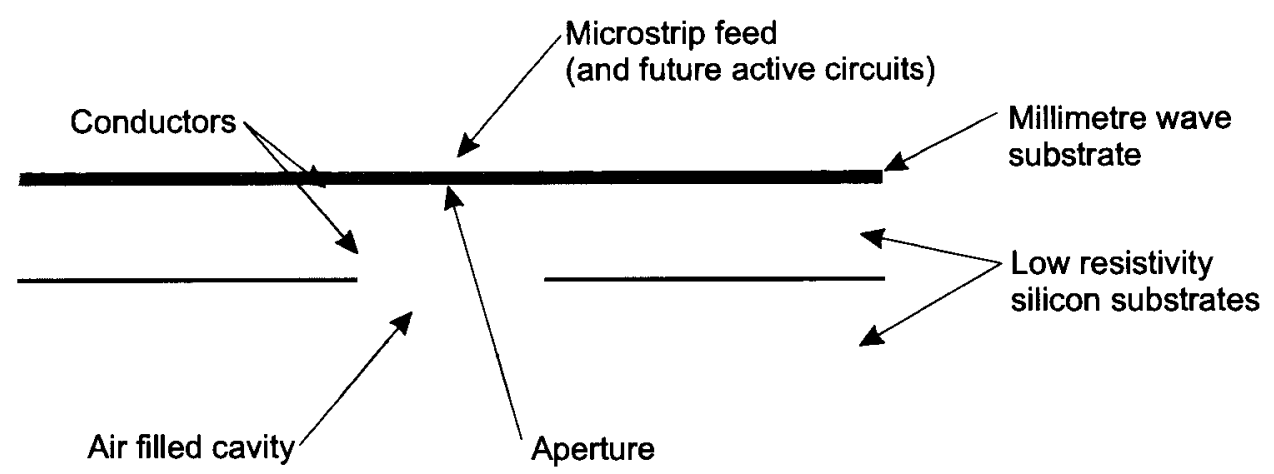

Figure 6.3: A possible micromachined spherical cavity resonator, although an actual cavity is likely to be more ellipsoidal.

Another possible item for future research is to try using direct wafer bonding to align and join the etched silicon wafer to a feed microstrip patterned on high-resistivity silicon. This procedure would remove the loss of the solder and also its extra thickness, which slightly lowers the resonant frequency of the cavity because of the extra volume the layer adds to the cavity. A higher quality factor might result if the lower conductivity and rougher solder layer is removed; however, perhaps not, if the dominating source of cavity loss remains the surface roughness of the upper edges of the etched cavities and not the solder.

Wafer bonding may be also useful if two hemispherical micromachined cavity halves are used to form a full spherical/ellipsoidal resonator. This geometry, shown in Figure 6.3, could improve the quality factor by over a factor of two compared to a hemispherical cavity, however, it may be difficult to align and bond (or solder) all three wafers. Furthermore, one of the resonator halves would have to be thinned to allow the feed to be mounted for aperture coupling such that no energy enters the lossy, low-resistivity silicon.

And finally, another possible item for future research is a mechanism to tune the resonator. The ability to tune the resonant frequency of the cavity would solve the size 
variation problem along with allowing the micromachined cavity to be used in a voltagecontrolled oscillator, assuming of course that the tuning is controlled by a voltage. The solder layer may also be useful to tune the cavity during fabrication. Since the solder thickness alters the resonant frequency of the etched cavity then perhaps adjusting the solder thickness a little, once the etched cavity size is known, can be used to compensate for some of the size (and hence frequency) variation that occured during the etching.

\subsection{Final Remarks}

Despite the suggestions for future development in the previous section, the current status of the micromachined hemispheroidal cavities is encouraging. The goal was to achieve a resonant frequency of around $77 \mathrm{GHz}$ while simultaneously maximizing both the loaded and unloaded quality factors. These results were achieved. Measurements of a prototype micromachined oblate hemispherical cavity resulted in a resonant frequency of $76.39 \mathrm{GHz}$, an unloaded quality factor of 1426 and a loaded quality factor of 909 . These micromachined resonators have a novel structure and a successfully developed, custom fabrication procedure. Additionally, the equations developed also predict these quantities thus allowing designers to quickly produce a cavity geometry than can be simulated successfully in a single iteration. And, satisfyingly, Figures 6.1 and 6.2 show that these prototype micromachined resonators have the best measured performance reported thus far at W-band. 


\section{Appendix A}

\section{Final Fabrication Procedures and Custom Photomasks}

The following masks were custom designed and used during the development of the cavities:

\begin{tabular}{|r|l|}
\hline Name & Purpose \\
\hline \hline SM2002-1 & Isotropic etching experiments. Vary etch mask size. \\
\hline SM2003-1 & Isotropic etching experiments. Vary etch mask size. \\
\hline SM2003-2 & Initial microstrip feed. Top of alumina. \\
\hline SM2003-3 & Initial microstrip feed. Bottom of alumina. Vary aperture size. \\
\hline SM2003-4 & Final Etch mask for cavities. \\
\hline SM2005-1 & Vialess transition test structures. \\
\hline SM2005-2 & Final microstrip feed. Top of alumina. \\
\hline SM2005-3 & Final indium solder layer pattern. \\
\hline SM2005-4 & Final microstrip feed. Bottom of alumina. Fixed aperture size. \\
\hline SM2005-5 & Final microstrip feed. Bottom of alumina. Vary aperture size. \\
\hline
\end{tabular}

These masks are shown on the following two pages. 
SM2002-1

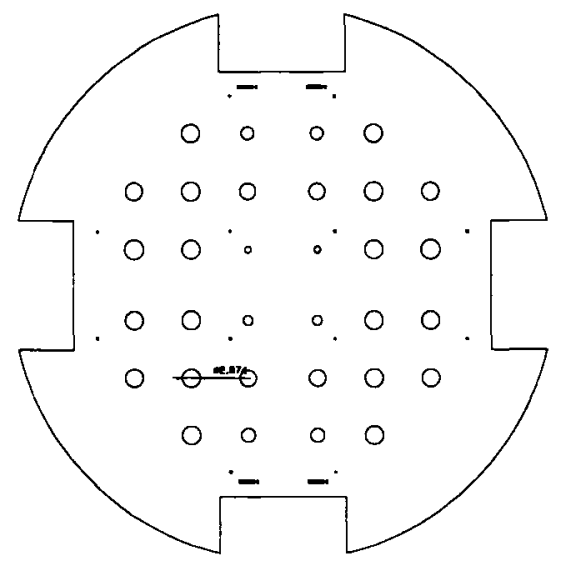

SM2003-2

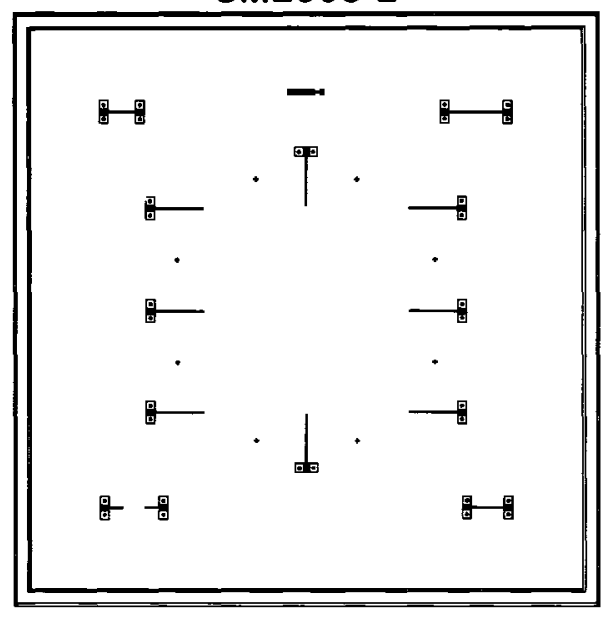

SM2003-4

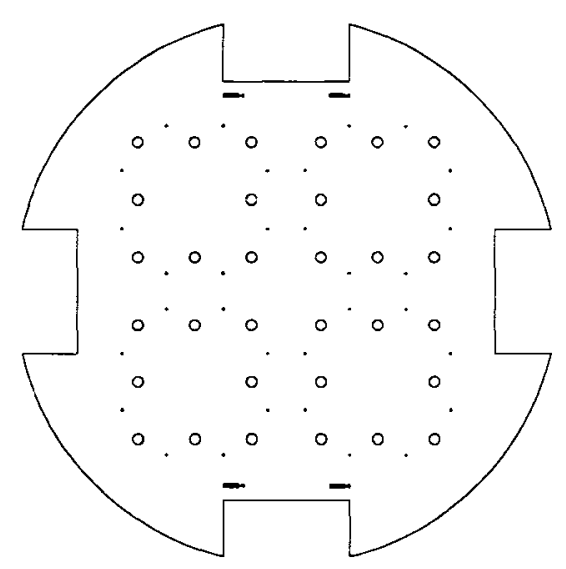

SM2003-1

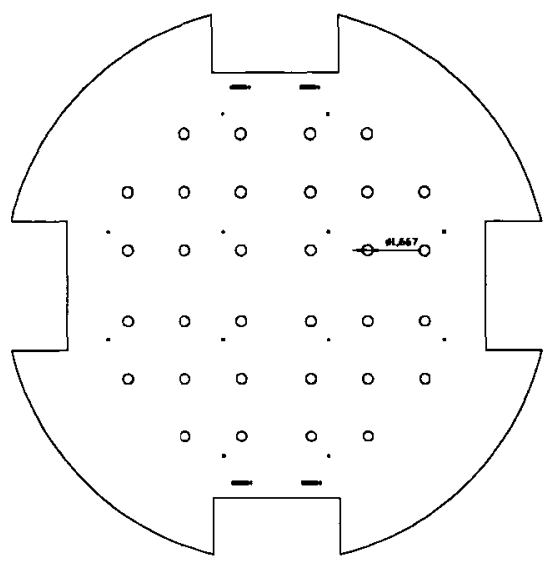

SM2003-3

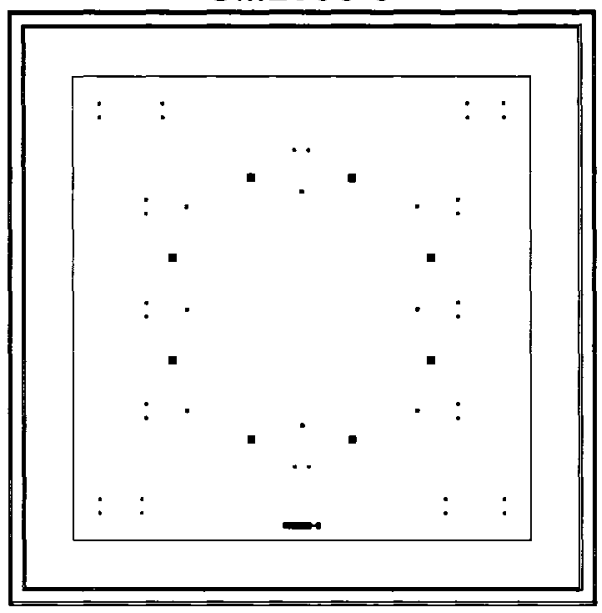

SM2005-1

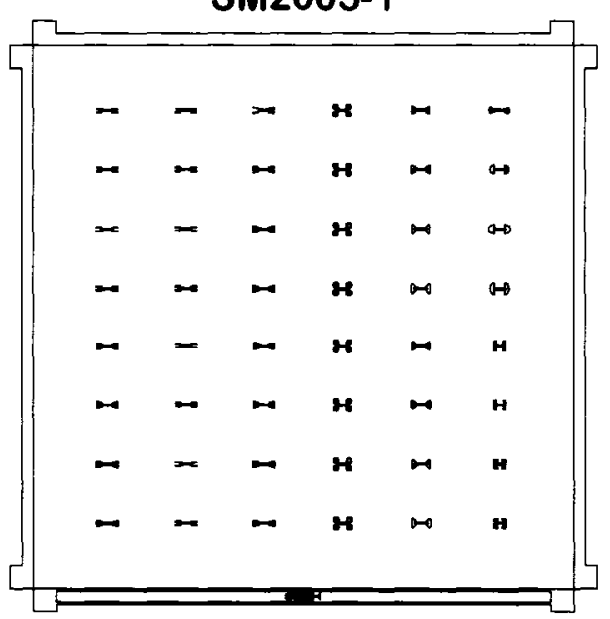




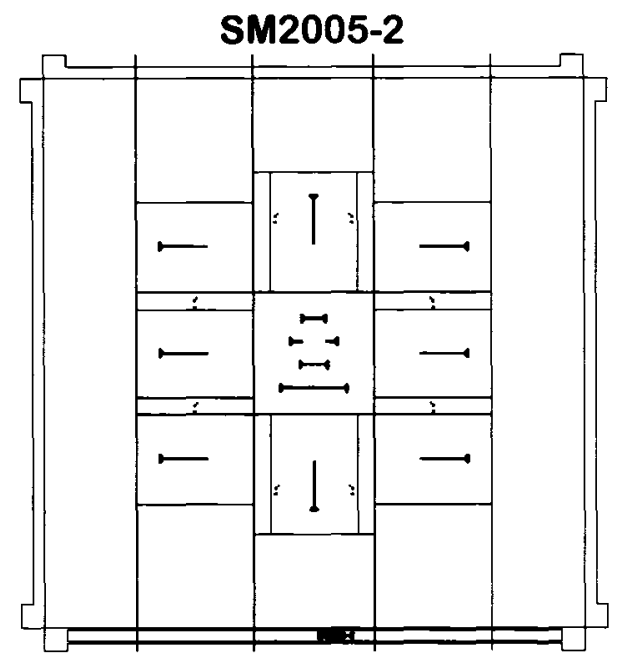

SM2005-4

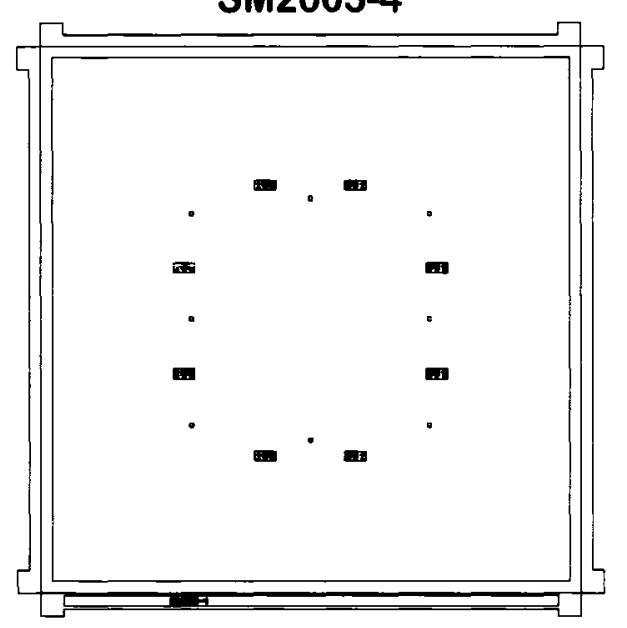

SM2005-3

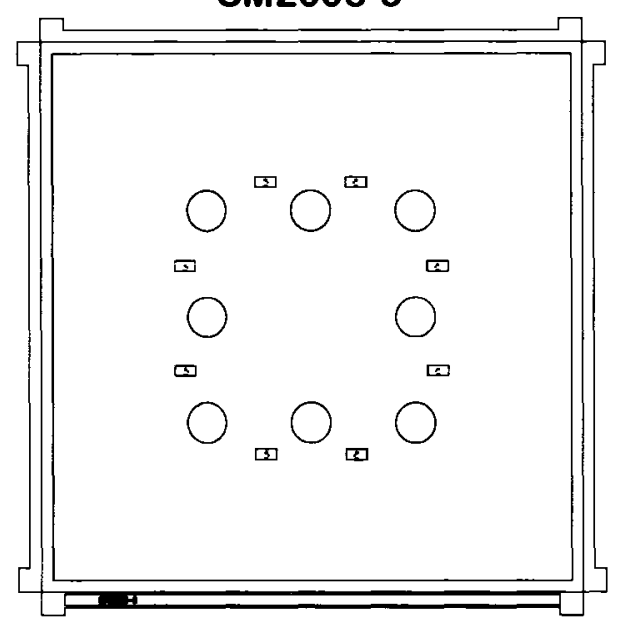

SM2005-5

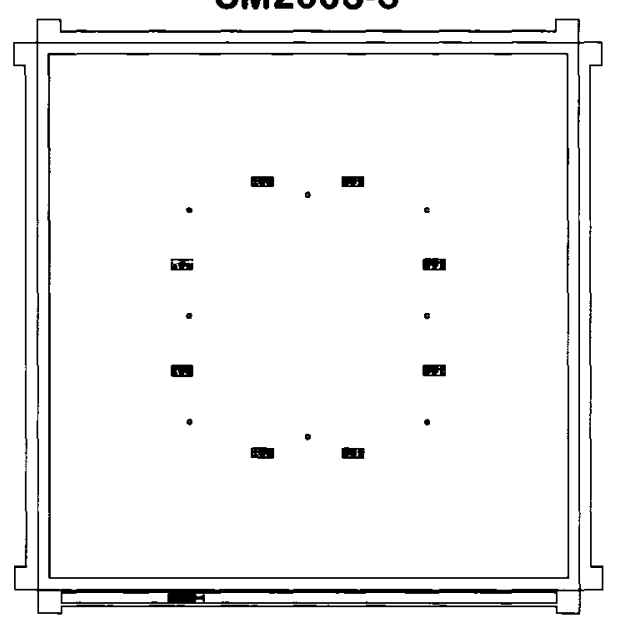




\section{Self-limited isotropic etching procedure for hemispherical cavities}

1. Select 1 silicon wafer, 3 inch diameter, $3 \mathrm{~mm}$ thick, CMOS grade, $\langle 100\rangle$ orientation, double side polished, $1 \Omega \mathrm{cm}$ resistivity (from Virginia Semiconductor)

2. Standard Clean

3. Deposit $2 \mu \mathrm{m} \mathrm{Si} \mathrm{N}_{4}$ by PECVD on both sides

4. Apply a $2.6 \mathrm{mil}(66.04 \mu \mathrm{m})$ thick polyethylene tape to top surface without air bubbles (tape is HNA etch mask and is commonly called a high performance mailing Tape, CS-22C, from MANCO Inc.)

5. Rinse in IPA, then dry $\left(30 \mathrm{~min}\right.$ at $\left.60^{\circ} \mathrm{C}\right)$

6. Evaporate $3000 \AA \mathrm{Al}$ on top of the tape (mask for the tape patterning)

7. Spin positive PR, pattern with mask "SM2003-4"

8. Etch the $\mathrm{Al}$

9. RIE the tape in Plasmatherm until holes are clean

(a) Process parameters: $50 \mathrm{sccm} \mathrm{O}_{2}$ at 100 mTorr, power: $100 \mathrm{~W}$ in RIE mode

(b) Etch sample for $2 \mathrm{hrs}$, rotate $135^{\circ}$, then 2 more hours, rotate and etch 2 more hrs for a total etch time of $6 \mathrm{hrs}$

10. Etch the Al mask completely off

11. Saw wafer into equal-sized quarters

12. For each wafer quarter

(a) Apply tape to each quarter wafer on the back, sides and front (covering only the alignment marks on the front) to protect wafer during etching

(b) Main HNA etch: $\mathrm{HF}: \mathrm{HNO}_{3}: \mathrm{CH}_{3} \mathrm{COOH}=2: 3: 3$ fresh HNA wet etch (use fresh etchant for each quarter!), $1: 45 \mathrm{hrs}$ at $72^{\circ} \mathrm{C}$, no agitation, flat, do not remove quarter during etch

(c) DI water rinse (30 min)

(d) Remove tape

(e) Clean the surfaces: acetone/IPA

(f) Etch the remaining top side $\mathrm{Si}_{3} \mathrm{~N}_{4}$ layer (Technics $\mathrm{CF}_{4}$ plasma)

(g) Sputter $300 \AA \mathrm{Ti}, 9700 \AA \mathrm{Au}$ on front side 


\section{Microstrip feed wafer fabrication procedure}

1. Select a TPS Superstrate Alumina substrate, $2 \times 2 \times 0.005$ inches

2. Std Clean

3. Back side process (first!)

(a) Sputter $2 \mu \mathrm{m} \mathrm{Au}$ on back side

(b) Pattern mask "SM2005-4"

(c) Etch Au

4. Front side process

(a) Pattern thick PR for liftoff, using mask "SM2005-2", soft-contact aligned to pattern on backside (best possible centreing of alignment marks using IR)

(b) Evaporate $1 \mu \mathrm{m} \mathrm{Au}$ on front side

(c) Liftoff

5. Check for pinholes in back side Au layer

6. Solder layer (third)

(a) Pattern NR7-1000 PR, using mask "SM2005-3"

(b) Cover front side with PR

(c) Electroplate $30 \mu \mathrm{m}$ of In: After cleaning the sample, use reverse pulse plating method at $0.050 \mathrm{~mA} / \mathrm{mm}^{2}$ maximum forward current and $0.025 \mathrm{~mA} / \mathrm{mm}^{2}$ maximum reverse current, in $99.9 \%$ Indium Sulphamate plating bath solution with agitation at room temperature

(d) Strip PR 


\section{References}

[1] D. Leeson, "A simple model of feedback oscillator noise spectrum," Proceedings of the IEEE, vol. 54, no. 2, pp. 329-330, Feb. 1966.

[2] R. Rhea and B. Clausen, "Recent trends in oscillator design," Microwave Journal, vol. 47, pp. 22-34, Jan. 2004.

[3] V. Radisic, L. Samoska, M. Micovic, M. Hu, P. Janke, C. Ngo, and L. Nguyen, " $80 \mathrm{GHz}$ MMIC HEMT VCO," IEEE Microwave and Wireless Components Letters, vol. 11, pp. 325-327, Aug. 2001.

[4] F. Lenk, M. Schott, J. Hilsenbeck, J. Wurfl, and W. Heinrich, "Low phase-noise monolithic GaInP/GaAs-HBT VCO for $77 \mathrm{GHz}$," IEEE MTT-S International Microwave Symposium Digest, 2003, vol. 2, pp. 903-906, June 2003.

[5] Z. Lao, J. Jensen, K. Guinn, and M. Sokolich, "80-GHz differential VCO in InP SHBTs," IEEE Microwave and Wireless Components Letters, vol. 14, pp. 407-409, Sept. 2004.

[6] H. Li, H.-M. Rein, and M. Schwerd, "SiGe VCOs operating up to $88 \mathrm{GHz}$, suitable for automotive radar sensors," Electronics Letters, vol. 39, pp. 1326-1327, Sept. 2003.

[7] R. Wanner, R. Lachner, and G. Olbrich, "SiGe integrated mm-wave push-push VCOs with reduced power consumption," IEEE Radio Frequency Integrated Circuits (RFIC) Symposium, 2006, p. 4, June 2006.

[8] H. Li, H.-M. Rein, R.-E. Makon, and M. Schwerd, "Wide-band VCOs in SiGe production technology operating up to about $70 \mathrm{GHz}$," IEEE Microwave and Wireless Components Letters, vol. 13, pp. 425-427, Oct. 2003.

[9] C. Cao and K. O, "Millimeter-wave voltage-controlled oscillators in 0.13- $\mu \mathrm{m}$ CMOS technology," IEEE Journal of Solid-State Circuits, vol. 41, pp. 1297-1304, June 2006.

[10] S. Hsu and H.-Z. Zhu, "W-band multiple-ring resonator by standard $0.18-\mu \mathrm{m}$ CMOS technology," IEEE Microwave and Wireless Components Letters, vol. 15, pp. 832-834, Dec. 2005.

[11] D. Pozar, Microwave Engineering, 2nd ed. New York: Wiley, 1998.

[12] X. Wu, I. Awai, Z. Yan, K. Wada, and T. Moriyoshi, "Quality factors of coplanar waveguide resonators," Asia Pacific Microwave Conference, 1999, vol. 3, pp. 670-673, Dec. 1999. 
[13] J.-H. Lee, S. Pinel, J. Papapolymerou, J. Laskar, and M. Tentzeris, "Low-loss LTCC cavity filters using system-on-package technology at $60 \mathrm{GHz}$," IEEE Transactions on Microwave Theory and Techniques, vol. 53, pp. 3817-3824, Dec. 2005.

[14] A. El-Tager and L. Roy, "Study of cylindrical multilayered ceramic resonators with rectangular air cavity for low-phase noise $\mathrm{K} / \mathrm{Ka}$-band oscillators," IEEE Transactions on Microwave Theory and Techniques, vol. 53, pp. 2211-2219, June 2005.

[15] L. Katehi, W. Chappell, S. Mohammadi, A. Margomenos, and M. Steer, "Heterogeneous wafer-scale circuit architectures," IEEE Microwave Magazine, vol. 8, pp. 52-69, Feb. 2007.

[16] W. Hansen, "A type of electric resonator," Journal of Applied Physics, vol. 9, pp. 654-663, Oct. 1938.

[17] R. Harrington, Time-Harmonic Electromagnetic Fields. New York: McGraw-Hill, 1961.

[18] J. Everard, "Low-noise power-efficient oscillators: Theory and design," IEE Proceedings Pt. G, vol. 133, no. 4, pp. 172-180, 1986.

[19] K. Petersen, "Silicon as a mechanical material," Proceedings of the IEEE, vol. 70, no. 5, pp. 420-457, May 1982.

[20] G. Kovacs, N. Maluf, and K. Petersen, "Bulk micromachining of silicon," Proceedings of the IEEE, vol. 86, no. 8, pp. 1536-1551, Aug. 1998.

[21] J. Bustillo, R. Howe, and R. Muller, "Surface micromachining for microelectromechanical systems," Proceedings of the IEEE, vol. 86, no. 8, pp. 1552-1574, Aug. 1998.

[22] C.-C. Nguyen, L. Katehi, and G. Rebeiz, "Micromachined devices for wireless communications," Proceedings of the IEEE, vol. 86, no. 8, pp. 1756-1768, Aug. 1998.

[23] J. Yao, "RF MEMS from a device perspective," Journal of Micromechanics and Microengineering, vol. 10, pp. R9-R38, June 2000.

[24] K. Herrick, J. Yook, and L. Katehi, "Microtechnology in the development of threedimensional circuits," IEEE Transactions on Microwave Theory and Techniques, vol. 46, no. 11, pp. 1832-1844, Nov. 1998.

[25] A. Yarbrough, R. Cole, and R. Robertson, "Development of released micromachined structures for millimeterwave device applications: preliminary results," Proceedings., IEEE Aerospace Conference, 1998., vol. 1, pp. 487-493, Mar. 1998.

[26] V. Lubecke, K. Mizuno, and G. Rebeiz, "Micromachining for terahertz applications," IEEE Transactions on Microwave Theory and Techniques, vol. 46, no. 11, pp. 1821-1831, Nov. 1998.

[27] R. Ribas, N. Bennouri, J. Karam, and B. Courtois, "GaAs MEMS design using 0.2 $\mu \mathrm{m}$ HEMT MMIC technology," Technical Digest 1997., 19th Annual Gallium Arsenide Integrated Circuit (GaAs IC) Symposium, 1997., pp. 127-130, Oct. 1997. 
[28] J. Long, "Sige radio frequency ICs for low-power portable communication," Proceedings of the IEEE, vol. 93, pp. 1598-1623, Sept. 2005.

[29] T. Lee and S. Wong, "CMOS RF integrated circuits at $5 \mathrm{GHz}$ and beyond," Proceedings of the IEEE, vol. 88, no. 10, pp. 1560-1571, Oct. 2000.

[30] T. Dickson, M.-A. LaCroix, S. Boret, D. Gloria, R. Beerkens, and S. Voinigescu, "30$100-\mathrm{GHz}$ inductors and transformers for millimeter-wave (Bi)CMOS integrated circuits," IEEE Transactions on Microwave Theory and Techniques, vol. 53, pp. 123-133, Jan. 2005.

[31] R. Howe, "Applications of silicon micromachining to resonator fabrication," 48th., Proceedings of the 1994 IEEE International Frequency Control Symposium, 1994., pp. 2-7, June 1994.

[32] J. Clark, W.-T. Hsu, M. Abdelmoneum, and C.-C. Nguyen, "High-Q UHF micromechanical radial-contour mode disk resonators," Microelectromechanical Systems, Journal of, vol. 14, pp. 1298-1310, Dec. 2005.

[33] K. Wang, Y. Yu, A.-C. Wong, and C.-C. Nguyen, "VHF free-free beam high-Q micromechanical resonators," MEMS '99. Twelfth IEEE International Conference on Micro Electro Mechanical Systems, 1999., pp. 453-458, Jan. 1999.

[34] K. Wang, A.-C. Wong, and C.-C. Nguyen, "VHF free-free beam high-Q micromechanical resonators," Microelectromechanical Systems, Journal of, vol. 9, no. 3, pp. 347-360, Sept. 2000 .

[35] J. R. Clark, W.-T. Hsu, and C. T.-C. Nguyen, "Measurement techniques for capacitivelytransduced VHF-to-UHF micromechanical resonators," Digest of Technical Papers, the 11 th Int. Conf. on Solid-State Sensors 8 Actuators (Transducers '01), pp. 1118-1121, June 2001.

[36] T. Roessig, R. Howe, A. Pisano, and J. Smith, "Surface-micromachined $1 \mathrm{MHz}$ oscillator with low-noise Pierce configuration," Solid-State Sensor and Actuator Workshop, Hilton Head Island, SC, June 1998.

[37] S. Lee, M. U. Demirci, , and C. T.-C. Nguyen, "A 10-MHz micromechanical resonator Pierce reference oscillator for communications," Digest of Technical Papers, the 11 th Int. Conf. on Solid-State Sensors \& Actuators (Transducers '01), pp. 1094-1097, June 2001.

[38] C.-C. Nguyen, "Vibrating RF MEMS for next generation wireless applications," Proceedings of the IEEE 2004 Custom Integrated Circuits Conference, 2004., pp. 257-264, Oct. 2004.

[39] J. Wang, J. Butler, T. Feygelson, and C.-C. Nguyen, "1.51-GHz nanocrystalline diamond micromechanical disk resonator with material-mismatched isolating support," 17th IEEE International Conference on. (MEMS) Micro Electro Mechanical Systems, 2004., pp. 641644, 2004. 
[40] S.-S. Li, Y.-W. Lin, Y. Xie, Z. Ren, and C.-C. Nguyen, "Micromechanical "hollow-disk" ring resonators," 17th IEEE International Conference on. (MEMS) Micro Electro Mechanical Systems, 2004., pp. 821-824, 2004.

[41] C. Nguyen, "Vibrating RF MEMS for low power wireless communications," Proceedings, 2000 Int. MEMS Workshop (MEMS '01), pp. 21-34, July 2001.

[42] C.-C. Nguyen, "Frequency-selective MEMS for miniaturized low-power communication devices," IEEE Transactions on Microwave Theory and Techniques, vol. 47, no. 8, pp. 1486-1503, Aug. 1999.

[43] C.-Y. Chi and G. Rebiez, "Planar microwave and millimeter-wave lumped elements and coupled-line filters using micro-machining techniques," IEEE Transactions on Microwave Theory and Techniques, vol. 43, no. 4, pp. 730-738, Apr. 1995.

[44] V. Lubecke, B. Barber, E. Chan, D. Lopez, and P. Gammel, "Self-assembling MEMS variable and fixed RF inductors," Asia-Pacific Microwave Conference, 2000, pp. 201204, Dec. 2000.

[45] L. Fan, R. Chen, A. Nespola, and M. Wu, "Universal MEMS platforms for passive RF components: suspended inductors and variable capacitors," MEMS 98. Proceedings., The Eleventh Annual International Workshop on Micro Electro Mechanical Systems, 1998., pp. 29-33, Jan. 1998.

[46] V. Malba, D. Young, J. Ou, A. Bernhardt, and B. Boser, "High-performance rf coil inductors on silicon," 48th IEEE Electronic Components and Technology Conference, 1998., pp. 252-255, May 1998.

[47] A. Dec and K. Suyama, "Micromachined varactor with wide tuning range," Electronics Letters, vol. 33, no. 11, pp. 922-924, May 1997.

[48] A. Dec and K. Suyama, "RF micromachined varactors with wide tuning range," IEEE MTT-S International Microwave Symposium Digest, 1998, vol. 1, pp. 357-360, June 1998.

[49] A. Dec and K. Suyama, "Micromachined electro-mechanically tunable capacitors and their applications to RF ICs," IEEE Transactions on Microwave Theory and Techniques, vol. 46, no. 12, pp. 2587--2596, Dec. 1998.

[50] Z. Feng, W. Zhang, B. Su, K. Harsh, K. Gupta, V. Bright, and Y. Lee, "Design and modeling of RF MEMS tunable capacitors using electro-thermal actuators," IEEE MTTS International Microwave Symposium Digest, 1999, vol. 4, pp. 1507-1510, June 1999.

[51] W. Wu, F. Huang, Y. Li, S. Zhang, X. Han, Z. Li, Y. Hao, and Y. Wang, "Rf inductors with suspended and copper coated thick crystalline silicon spirals for monolithic MEMS LC circuits," IEEE Microwave and Wireless Components Letters, vol. 15, pp. 853-855, Dec. 2005.

[52] H.-C. Chen, C.-H. Chien, H.-W. Chiu, S.-S. Lu, K.-N. Chang, K.-Y. Chen, and S.H. Chen, "A low-power low-phase-noise LC VCO with MEMS Cu inductors," IEEE Microwave and Wireless Components Letters, vol. 15, pp. 434-436, June 2005. 
[53] E.-C. Park, Y.-S. Choi, J.-B. Yoon, S. Hong, and E. Yoon, "Fully integrated low phasenoise VCOs with on-chip MEMS inductors," IEEE Transactions on Microwave Theory and Techniques, vol. 51, pp. 289-296, Jan. 2003.

[54] S. Pinel, F. Cros, S. Nuttinck, S.-W. Yoon, M. Allen, and J. Laskar, "Very high-Q inductors using RF-MEMS technology for system-on-package wireless communication integrated module," IEEE MTT-S International Microwave Symposium Digest, 2003, vol. 3, pp. 1497-1500, June 2003.

[55] V. Lubecke, B. Barber, E. Chan, D. Lopez, M. Gross, and P. Gammel, "Self-assembling MEMS variable and fixed RF inductors," IEEE Transactions on Microwave Theory and Techniques, vol. 49, pp. 2093-2098, Nov. 2001.

[56] S. Chang and S. Sivoththaman, "A tunable RF MEMS inductor on silicon incorporating an amorphous silicon bimorph in a low-temperature process," IEEE Electron Device Letters, vol. 27, pp. 905 907, Nov. 2006.

[57] T. Weller, K. Herrick, and L. Katehi, "Quasi-static design technique for MM-wave micromachined filters with lumped elements and series stubs," IEEE Transactions on Microwave Theory and Techniques, vol. 45, no. 6, pp. 931-938, June 1997.

[58] K. Takahashi, S. Fujita, U. Sangawa, A. Ono, T. Urabe, S. Takeyama, H. Ogura, and $\mathrm{H}$. Yabuki, "K-band receiver front-end IC integrating micromachined filter and flip-chip assembled active devices," IEEE MTT-S International Microwave Symposium Digest, 1999, vol. 1, pp. 229-232, June 1999.

[59] C.-Y. Chi and G. Rebeiz, "Conductor-loss limited stripline resonator and filters," IEEE Transactions on Microwave Theory and Techniques, vol. 44, no. 4, pp. 626 630, Apr. 1996.

[60] P. Blondy, A. Brown, D. Crost, and G. Rebeiz, "Low loss micromachined filters for millimeter-wave telecommunication systems," IEEE MTT-S International Microwave Symposium Digest, 1998, vol. 3, pp. 1181-1184, June 1998.

[61] A. Brown, P. Blondy, and G. Rebeiz, "Microwave and millimeter-wave high Q micromachined resonators," International Journal of RF and Microwave Computer-Aided Engineering, vol. 9, no. 4, pp. 326-337, July 1999.

[62] A. Brown and G. Rebeiz, "A Ka-band micromachined low-phase-noise oscillator," IEEE Transactions on Microwave Theory and Techniques, vol. 47, no. 8, pp. 1504-1508, Aug. 1999.

[63] A. Brown, P. Blondy, and G. Rebeiz, "Microwave and millimeter-wave high Q micromachined resonators," 29th European Microwave Conference, 1999., pp. 407-410, Oct. 1999.

[64] I. Llamas-Garro, Y. Kim, C.-W. Baek, and Y.-K. Kim, "A planar high-Q micromachined monolithic half-coaxial transmission-line filter," IEEE Transactions on Microwave Theory and Techniques, vol. 54, pp. 4161-4168, Dec. 2006. 
[65] J. Reid, E. Marsh, and R. Webster, "Micromachined rectangular-coaxial transmission lines," IEEE Transactions on Microwave Theory and Techniques, vol. 54, pp. 3433 3442, Aug. 2006.

[66] J. Papapolymerou, J.-C. Cheng, J. East, and L. Katehi, "A micromachined high-Q Xband resonator," IEEE Microwave and Guided Wave Letters, vol. 7, no. 6, pp. 168-170, June 1997.

[67] L. Harle and L. Katehi, "A silicon micromachined four-pole linear phase filter," IEEE Transactions on Microwave Theory and Techniques, vol. 52, pp. 1598-1607, June 2004.

[68] M. Hill, J. Papapolymerou, and R. Ziolkowski, "High-Q micromachined resonant cavities in a K-band diplexer configuration," Microwaves, Antennas and Propagation, IEE Proceedings -, vol. 148, pp. 307-312, Oct. 2001.

[69] Y. Kwon, C. Cheon, N. Kim, C. Kim, I. Song, and C. Song, "A Ka-band MMIC oscillator stabilized with a micromachined cavity," IEEE Microwave and Guided Wave Letters, vol. 9, no. 9, pp. 360-362, Sept. 1999.

[70] I. Song, C. Kim, Y. Kwon, C. Cheon, and C. Song, "Phase noise enhancement of GaAs high electron mobility transistors using micromachined cavity resonators at Ka-band," Japanese Journal of Applied Physics, vol. 38, no. 6A/B, pp. L601-L602, June 1999.

[71] C. Kim, I. Song, C. Song, C. Cheon, Y. Kwon, and S. Lee, "A micromachined cavity resonator for millimeter-wave oscillator applications," Sensors and Actuators A, vol. 83, pp. 1-5, July 2000 .

[72] D. Mercier, M. Chatras, J. Orlianges, C. Champeaux, A. Catherinot, P. Blondy, D. Cros, and J. Papapolymerou, "A micromachined tunable cavity resonator," 33rd European Microwave Conference, 2003., vol. 2, pp. 675-677, Oct. 2003.

[73] K.-J. Song, B.-S. Yoon, J.-C. Lee, B. LEE, J.-H. Kim, N.-Y. Kim, J.-Y. Park, G.-H. Ho, and J.-U. Bu, "A micromachined millimeter-wave cavity resonator on silicon and quartz substrates," Japanese Journal of Applied Physics, vol. 40, p. L1394 L1397, Dec. 2001.

[74] R. H. C. Tavernier and J. Papapolymerou, "A hybrid micromachined high-Q cavity resonator at $5.8 \mathrm{GHz}$," 30th European Microwave Conference, 2000., pp. 125-128, Oct. 2000.

[75] C. Tavernier, R. Henderson, and J. Papapolymerou, "A reduced-size silicon micromachined high-Q resonator at $5.7 \mathrm{GHz}$," IEEE Transactions on Microwave Theory and Techniques, vol. 50, no. 10, pp. 2305-2314, Oct. 2002.

[76] X. Gong, A. Margomenos, B. Liu, S. Hajela, L. Katehi, and W. Chappell, "Precision fabrication techniques and analysis on high-Q evanescent-mode resonators and filters of different geometries," IEEE Transactions on Microwave Theory and Techniques, vol. 52, pp. 2557-2566, Nov. 2004.

[77] Y. Wu, Q. Chen, V. Fusco, M. Zheng, and P. Hall, "Radiation leakage from an under metallized silicon cavity," IEEE MTT-S International Microwave Symposium Digest, 1999, vol. 3, pp. 1331-1334, June 1999. 
[78] H. Wheeler, "Formulas for the skin effect," Proceedings of the IRE, vol. 30, no. 9, pp. $412-424$, Sept. 1942.

[79] P. Waldow and I. Wolff, "The skin-effect at high frequencies," IEEE Transactions on Microwave Theory and Techniques, vol. 33, no. 10, pp. 1076-1082, Oct. 1985.

[80] P. Wu and Z. Yu, "A micromechanical high-q resonator based on hybrid cavity" APMC 2005. Asia-Pacific Conference Proceedings Microwave Conference Proceedings, 2005., vol. 2, p. 3, Dec. 2005.

[81] M. Stickel, G. Eleftheriades, and P. Kremer, "High-Q bulk micromachined silicon cavity resonator at Ka-band," Electronics Letters, vol. 37, no. 7, pp. 433-435, Mar. 2001.

[82] M. Stickel, P. Kremer, and G. Eleftheriades, "High-Q microstrip-fed bulk micromachined silicon cavities," IEEE Antennas and Propagation Society International Symposium, 2003., vol. 2, pp. 632-635, June 2003.

[83] M. Stickel, P. Kremer, and G. Eleftheriades, "High-Q silicon micromachined cavity resonators at $30 \mathrm{GHz}$ using the split-block technique," Microwaves, Antennas and Propagation, IEE Proceedings -, vol. 151, pp. 450-454, Oct. 2004.

[84] K. Strohm, F. Schmuckle, B. Schauwecker, J.-F. Luy, and W. Heinrich, "Silicon micromachined RF MEMS resonators," IEEE MTT-S International Microwave Symposium Digest, 2002, vol. 2, pp. 1209-1212, June 2002.

[85] K. Strohm, F. Schmuckle, O. Yaglioglu, J.-F. Luy, and W. Heinrich, "3D silicon micromachined RF resonators," IEEE MTT-S International Microwave Symposium Digest, 2003, vol. 3, pp. 1801-1804, June 2003.

[86] N. Faure-Muret, D. Baillargeat, S. Verdeyme, J. GaliLre, E. Estebe, J.-P. Ghesquiers, and F. Bernard, "A high-Q quasi-planar filtering solution for 60-GHz applications," IEEE Microwave and Wireless Components Letters, vol. 15, pp. 354-356, May 2005.

[87] G. Mie, "Beiträge zur optik trüber medien, speziall kulloidaler metallösungen," Annalen der Physik, vol. 25, no. 3, pp. 337-445, 1908.

[88] K. Kurokawa, "The expansions of electromagnetic fields in cavities," IEEE Transactions on Microwave Theory and Techniques, vol. 6, no. 2, pp. 178-187, Apr. 1958.

[89] W. Barrow and W. Mieher, "Natural oscillations of electrical cavity resonators," Proceedings of the IRE, vol. 28, no. 4, pp. 184-191, Apr. 1940.

[90] C. Lee, S. Lee, and S. Chuang, "Plot of modal field distribution in rectangular and circular waveguides," IEEE Transactions on Microwave Theory and Techniques, vol. 33, no. 3, pp. 271-274, Mar. 1985.

[91] P. Bhartia and M. Hamid, "Eigenvalues for a spherical cavity with an impedance wall (Correspondence)," IEEE Transactions on Microwave Theory and Techniques, vol. 19, no. 1, pp. 110-111, Jan. 1971. 
[92] M. Jouget, "Natural electromagnetic oscillations of a spherical cavity," Science Abstracts, vol. 42A, no. 3822, 1939.

[93] T. Owe Berg, "Elementary theory of the spherical cavity resonator," Wireless Engineer, vol. 18 , no. 1843 , p. $287,1941$.

[94] M. Gastine, L. Courtois, and J. Dormann, "Electromagnetic resonances of free dielectric spheres," IEEE Transactions on Microwave Theory and Techniques, vol. 15, no. 12, pp. 694-700, Dec. 1967.

[95] P. Affolter and B. Eliasson, "Electromagnetic resonances and Q-factors of lossy dielectric spheres," IEEE Transactions on Microwave Theory and Techniques, vol. 21, no. 9, pp. 573-578, Sept. 1973.

[96] A. Julien and P. Guillon, "Electromagnetic analysis of spherical dielectic shielded resonators (Short paper)," IEEE Transactions on Microwave Theory and Techniques, vol. 34, no. 6, pp. 723-729, June 1986.

[97] K. Leung, "Dimensional analysis of two-layer spherical dielectric resonator," IEEE Microwave and Guided Wave Letters, vol. 10, no. 4, pp. 139-141, Apr. 2000.

[98] R. Ghose, "Excitation of higher order modes in spherical cavities," IEEE Transactions on Microwave Theory and Techniques, vol. 5, no. 1, pp. 18-22, Jan. 1957.

[99] H. Thal, "Cylindrical $\mathrm{TE}_{011} / \mathrm{TM}_{111}$ mode control by cavity shaping," IEEE Transactions on Microwave Theory and Techniques, vol. 27, no. 12, pp. 982 986, Dec. 1979.

[100] H. Bethe, "Theory of diffraction by small holes," Physical Review, vol. 66, no. 7 \& 8, pp. 163-182, Oct. 1944.

[101] N. Marcuvitz, Waveguide Handbook, 1st ed. New York: McGraw-Hill, 1951.

[102] R. Collin, Feild Theory of Guided Waves, 1st ed. New York: McGraw-Hill, 1960.

[103] M. Madou, Fundamentals of Microfabrication. Boca Raton, Florida: CRC Press, 1997.

[104] 1501 Powhatan Street, Fredericksburg, Virginia, USA. [Online]. Available: www.virginiasemi.com

[105] K. Williams and R. Muller, "Etch rates for micromachining processing," Microelectromechanical Systems, Journal of, vol. 5, no. 4, pp. 256-269, Dec. 1996.

[106] K. Williams, K. Gupta, and M. Wasilik, "Etch rates for micromachining processing-part II," Microelectromechanical Systems, Journal of, vol. 12, no. 6, pp. 761-778, Dec. 2003.

[107] H. Robbins and B. Schwartz, "Chemical etching of silicon I: The system $\mathrm{HF}, \mathrm{HNO}_{3}$ and $\mathrm{H}_{2} \mathrm{O}$," Journal of The Electrochemical Society, vol. 106, no. 6, pp. 505-508, June 1959.

[108] H. Robbins and B. Schwartz, "Chemical etching of silicon II: The system $\mathrm{HF}, \mathrm{HNO}_{3}, \mathrm{H}_{2} \mathrm{O}$ and $\mathrm{HC}_{2} \mathrm{H}_{3} \mathrm{O}_{2}$," Journal of The Electrochemical Society, vol. 107, no. 2, pp. 108-111, Feb. 1960. 
[109] H. Robbins and B. Schwartz, "Chemical etching of silicon III: Temperature study in the acid system," Journal of The Electrochemical Society, vol. 108, no. 4, pp. 365-372, Apr. 1961.

[110] H. Robbins and B. Schwartz, "Chemical etching of silicon IV: Etching technology," Journal of The Electrochemical Society, vol. 123, no. 12, pp. 1903-1909, Dec. 1976.

[111] W. Kern, "Chemical etching of silicon, germanium, gallium arsenide and gallium phosphide," RCA Review, vol. 39 Part 2, pp. 278-308, June 1978.

[112] C.-H. Han and E. S. Kim, "Fabrication of dome-shaped diaphragm with circular clamped boundary on silicon substrate," MEMS '99. Twelfth IEEE International Conference on Micro Electro Mechanical Systems, 1999., pp. 505-510, Jan. 1999.

[113] K. Wise, T. Jackson, N. Masnari, and M. Robinson, "Fabrication of hemispherical structures using semiconductor technology for use in thermonuclear fusion research," Journal of Vacuum Science and Technology, vol. 16, no. 3, pp. 936-939, May 1979.

[114] W. Runyan and K. Bean, Semiconductor integrated circuit processing technology. Reading, MA: Addison-Wesley, 1990, p. 250.

[115] H. Hashimoto, S. Tanaka, and K. Sato, "Silicon acoustic lens for scanning acoustic microscope (SAM)," International Conference on Solid-State Sensors and Actuators, 1991. Digest of Technical Papers, TRANSDUCERS '91., 1991, pp. 853-859, June 1991.

[116] F. Tischer, "Excess surface resistance due to surface roughness at $35 \mathrm{GHz}$," IEEE Transactions on Microwave Theory and Techniques, vol. 22, no. 5, pp. 566-569, May 1974.

[117] A. Chandra Kundu and I. Awai, "Resonant frequency and quality factors of a silvercoated $\frac{\lambda}{4}$ dielectric waveguide resonator," IEEE Transactions on Microwave Theory and Techniques, vol. 46, pp. 1124-1131, Aug. 1998.

[118] F. Tischer, "Surface characteristics of metals and waveguide attenuation at millimeterwave frequencies between 25 and $160 \mathrm{GHz}$," Microwave Symposium Digest, MTT-S International, vol. 76, pp. 238-240, June 1976.

[119] S. Hatano and F. Nihei, "Measurement of surface resistance in oversized circular waveguide at millimeter wavelengths," IEEE Transactions on Microwave Theory and Techniques, vol. 24, pp. 886-887, Nov. 1976.

[120] S. P. Morgan, "Effect of surface roughness on eddy-current losses at microwave frequencies," Journal of Applied Physics, vol. 20, p. 352 362, 1949.

[121] F. Tischer, "Excess conduction losses at millimeter wavelengths," IEEE Transactions on Microwave Theory and Techniques, vol. 24, no. 11, pp. 853-858, Nov. 1976.

[122] Y.-C. Wang, "The screening potential theory of excess conduction loss at millimeter and submillimeter wavelengths," IEEE Transactions on Microwave Theory and Techniques, vol. 26, no. 11, pp. 858 861 , Nov. 1978. 
[123] J. Rautio, "An investigation of microstrip conductor loss," IEEE Microwave Magazine, vol. 1, no. 4, pp. 60-67, Dec. 2000.

[124] S. Lucyszyn, "Investigation of wang's model for room-temperature conduction losses in normal metals at terahertz frequencies," IEEE Transactions on Microwave Theory and Techniques, vol. 53, no. 4, pp. 1398-1403, Apr. 2005.

[125] S. Lucyszyn, "Investigation of anomalous room temperature conduction losses in normal metals at terahertz frequencies," Microwaves, Antennas and Propagation, IEE Proceedings -, vol. 151, pp. 321-329, Aug. 2004.

[126] S. Groiss, I. Bardi, O. Biro, K. Preis, and K. Richter, "Parameters of lossy cavity resonators calculated by the finite element method," IEEE Transactions on Magnetics, vol. 32, pp. 894-897, May 1996.

[127] R. Hoffmann, Handbook of microwave integrated circuits. Norwood, MA: Artech House, 1987.

[128] "Surface roughness," Ansoft HFSS Help, Ansoft Corporation.

[129] Z. Ma, D. Klymyshyn, S. Achenbach, and J. Mohr, "LIGA cavity resonator for K-band applications," Proceedings. 2005 International Conference on MEMS, NANO and Smart Systems, 2005., pp. 106-109, July 2005.

[130] C. Holloway and E. Kuester, "Power loss associated with conducting and superconducting rough interfaces," IEEE Transactions on Microwave Theory and Techniques, vol. 48, no. 10 , pp. $1601-1610$, Oct. 2000.

[131] C. Butler, Y. Rahmat-Samii, and R. Mittra, "Electromagnetic penetration through apertures in conducting surfaces," IEEE Transactions on Antennas and Propagation, vol. 26, no. 1, pp. 82-93, Jan. 1978.

[132] G. Painchaud, "A novel microstrip to cylindrical cavity transistion and its application in the design of highly stable $12 \mathrm{GHz}$ GaAs FET oscillators," M.A.Sc. Thesis, University of Ottawa, 1976.

[133] D. James, G. Painchaud, and W. Hoefer, "Aperture coupling between microstrip and resonant cavities," IEEE Transactions on Microwave Theory and Techniques, vol. 25, no. 5, pp. 392-396, May 1977.

[134] H. Wheeler, "Coupling holes between resonant cavities or wave-guides evaluated in terms of volume ratios," IEEE Transactions on Microwave Theory and Techniques, vol. 12, no. 2, pp. $231-244$, Mar. 1964.

[135] W. Hoefer and D. James, "Microstrip to waveguide coupling through holes," 5th Colloquim on Microwave Communication, pp. 221-231, June 1974.

[136] S. Cohn, "Determination of aperture parameters by electrolytic-tank measurements," Prceedings of the IRE, pp. 1416-1421, Nov. 1951. 
[137] S. Cohn, "The electric polarizability of apertures of arbitrary shape," Prceedings of the IRE, pp. 1069-1071, Sept. 1952.

[138] F. De Meulenaere and J. Van Bladel, "Polarizability of some small apertures," IEEE Transactions on Antennas and Propagation, vol. 25, no. 2, pp. 198-205, Mar. 1977.

[139] R. De Smedt and J. Van Bladel, "Magnetic polarizability of some small apertures," IEEE Transactions on Antennas and Propagation, vol. 28, no. 5, pp. 703-707, Sept. 1980.

[140] V. Fabrikant, "Magnetic polarizability of small apertures: Analytical approach," Journal of Physics A: Mathematical and General, vol. 20, pp. 323-338, 1987.

[141] E. Okon and R. Harrington, "The polarizabilities of electrically small apertures of arbitrary shape," IEEE Transactions on Electromagnetic Compatibility, vol. 23, no. 4, pp. 359-366, Nov. 1981.

[142] R. Yang and A. Omar, "Analysis of thin inclined rectangular aperture with arbitrary location in rectangular waveguide," IEEE Transactions on Microwave Theory and Techniques, vol. 41, no. 8, pp. 1461-1463, Aug. 1993.

[143] D. Kreinheder and T. Lingren, "Improved selectivity in cylindrical $\mathrm{TE}_{011}$ filters by $\mathrm{TE}_{211} / \mathrm{TE}_{311}$ mode control," IEEE Transactions on Microwave Theory and Techniques, vol. 82, no. 9, pp. 1383-1387, Sept. 1982.

[144] C. Montgomery, R. Dicke, and E. Purcell, Principles of Microwave Circuits. New York: McGraw Hill, 1948.

[145] J.-S. Hong and M. Lancaster, Microstrip Filters for RF/Microwave Applications. United States: Wiley, 2001.

[146] T. Itoh, Numerical Techniques for Microwave and Millimeter-Wave Passive Structures. United States: Wiley, 1989.

[147] I. Wolff, G. Kompa, and R. Mehran, "Calculation method for microstrip discontinuities and T junctions," Electronics Letters, vol. 8, no. 7, pp. 177-179, Apr. 1972.

[148] G. Kompa and R. Mehran, "Planar waveguide model for calculating microstrip components," Electronics Letters, vol. 11, no. 19, pp. 459-460, Sept. 1975.

[149] E. Hammerstad and O. Jensen, "Accurate models for microstrip computer-aided design," MTT-S International Microwave Symposium Digest, 1980, vol. 80, no. 1, pp. 407-409, May 1980.

[150] R. Collin, Foundations for Microwave Engineering, 2nd ed. United States: McGraw-Hill, 1992.

[151] M. Kirschning and R. Jansen, "Accurate model for effective dielectric constant of microstrip with validity up to millimetre-wave frequencies," Electronics Letters, vol. 18, no. 6 , pp. 272-273, Mar. 1982. 
[152] M. Kobayashi, "A dispersion formula satisfying recent requirements in microstrip CAD," IEEE Transactions on Microwave Theory and Techniques, vol. 36, no. 8, pp. 1246-1250, Aug. 1988.

[153] D. Kajfez and E. Hwan, "Q-Factor measurement with network analyzer," IEEE Transactions on Microwave Theory and Techniques, vol. 32, no. 7, pp. 666-670, July 1984.

[154] D. Kajfez, "Q factor measurements, analog and digital," Workshop on The Q-Factor Analysis and Measurement, 2000 Asia-Pacific Microwave Conference, Dec. 2000.

[155] E.-Y. Sun and S.-H. Chao, "Unloaded Q measurement-the critical points method," IEEE Transactions on Microwave Theory and Techniques, vol. 43, no. 8, pp. 1983-1986, Aug. 1995.

[156] R. Kwok and J.-F. Liang, "Characterization of high-Q resonators for microwave filter applications," IEEE Transactions on Microwave Theory and Techniques, vol. 47, no. 1, pp. 111-114, Jan. 1999.

[157] J. Drozd and W. Joines, "Determining Q using S parameter data," IEEE Transactions on Microwave Theory and Techniques, vol. 44, no. 11, pp. 2123-2127, Nov. 1996.

[158] J. Bray and L. Roy, "Measuring the unloaded, loaded, and external quality factors of one- and two-port resonators using scattering-parameter magnitudes at fractional power levels," Microwaves, Antennas and Propagation, IEE Proceedings -, vol. 151, pp. 345-350, Aug. 2004.

[159] E. L. Ginzton, Microwave Measurements. New York: McGraw-Hill, 1957, Chapter 9.

[160] E. Ginzton, "Microwave Q measurements in the presence of coupling losses," IEEE Transactions on Microwave Theory and Techniques, vol. 6, no. 4, pp. 383-389, Oct. 1958.

[161] D. Kajfez, "Graphical analysis of Q circuits (Correspondence)," IEEE Transactions on Microwave Theory and Techniques, vol. 11, no. 5, pp. 453-454, Sept. 1963.

[162] D. Kajfez, "Linear fractional curve fitting for measurement of high Q factors," IEEE Transactions on Microwave Theory and Techniques, vol. 42, no. 7, pp. 1149-1153, July 1994.

[163] D. Kajfez, "Random and systematic uncertainties of reflection-type Q-factor measurement with network analyzer," IEEE Transactions on Microwave Theory and Techniques, vol. 51, no. 2, pp. 512-519, Feb. 2003.

[164] K. Hjort, J.-A. Schweitz, and B. Hok, "Bulk and surface micromachining of GaAs structures," 'Proceedings, An Investigation of Micro Structures, Sensors, Actuators, Machines and Robots'. , IEEE Micro Electro Mechanical Systems, 1990., pp. 73-76, Feb. 1990.

[165] H. Hashimoto, S. Tanaka, K. Sato, I. Ishikawa, S. Kato, and N. Chubachi, "Chemical isotropic etching of single-crystal silicon for acoustic lens of scanning acoustic microscope," Japanese Journal of Applied Physics, vol. 32, pp. 2543-2546, May 1993. 
[166] K. Wise and M. Robinson, "Solid-state processes to produce hemispherical components for intertial fusion targets," Journal of Vacuum Science and Technology, vol. 18, no. 3, pp. 1179-1182, Apr. 1981.

[167] C. Han and E. Kim, "Study of self-limiting etching behavior in wet isotropic etching of silicon," Japanese Journal of Applied Physics, vol. 37, pp. 6939-6941, Dec. 1998.

[168] G. Zheng, P. Kirby, A. Rodriguez, J. Papapolymerou, M. Tentzeris, and L. Dunleavy, "Design and on-wafer measurement of a W-band via-less CPW RF probe pad to microstrip transition," 33rd European Microwave Conference, 2003., vol. 1, pp. 443-446, Oct. 2003.

[169] J. Kassner and W. Menzel, "In-circuit testing of complex circuits using on-wafer probing and electromagnetic coupled ground interconnects," IEEE MTT-S International Microwave Symposium Digest., 2000, vol. 3, pp. 1863-1866, June 2000.

[170] M. Schmidt, "Wafer-to-wafer bonding for microstructure formation," Proceedings of the IEEE, vol. 86, no. 8, pp. 1575-1585, Aug. 1998.

[171] Q.-Y. Tong, G. Cha, R. Gafiteanu, and U. Gosele, "Low temperature wafer direct bonding," Microelectromechanical Systems, Journal of, vol. 3, no. 1, pp. 29-35, Mar. 1994.

[172] H. Quenzer, W. Benecke, and C. Dell, "Low temperature wafer bonding for micromechanical applications," Micro Electro Mechanical Systems, 1992, MEMS '92, Proceedings. 'An Investigation of Micro Structures, Sensors, Actuators, Machines and Robot'. IEEE, pp. 49-55, Feb. 1992.

[173] J. Becker, J. East, and L. Katehi, "Performance of silicon micromachined waveguide at W-band," Electronics Letters, vol. 38, pp. 638-639, June 2002. 